\title{
Invertibility of Frame Operators on Besov-Type Decomposition Spaces
}

\author{
José Luis Romero ${ }^{1,2} \cdot$ Jordy Timo van Velthoven ${ }^{1,3}$ (D) Felix Voigtlaender 4
}

Received: 13 May 2019 / Accepted: 26 January 2022 / Published online: 21 February 2022

(c) The Author(s) 2022

\begin{abstract}
We derive an extension of the Walnut-Daubechies criterion for the invertibility of frame operators. The criterion concerns general reproducing systems and Besov-type spaces. As an application, we conclude that $L^{2}$ frame expansions associated with smooth and fast-decaying reproducing systems on sufficiently fine lattices extend to Besov-type spaces. This simplifies and improves recent results on the existence of atomic decompositions, which only provide a particular dual reproducing system with suitable properties. In contrast, we conclude that the $L^{2}$ canonical frame expansions extend to many other function spaces, and, therefore, operations such as analyzing using the frame, thresholding the resulting coefficients, and then synthesizing using the canonical dual frame are bounded on these spaces.
\end{abstract}

Keywords Atomic decompositions · Banach frames - Besov-type decomposition space Canonical dual frame $\cdot$ Walnut-Daubechies representation · Frame operator · Generalized shift-invariant systems

Mathematics Subject Classification 42B35 - 42C15 - 42C40

\footnotetext{
Jordy Timo van Velthoven

jordy-timo.van-velthoven@univie.ac.at; j.t.vanvelthoven@tudelft.nl

José Luis Romero

jose.luis.romero@univie.ac.at

Felix Voigtlaender

felix.voigtlaender@ku.de; felix@voigtlaender.xyz

1 Faculty of Mathematics, University of Vienna, Oskar-Morgenstern-Platz 1, 1090 Vienna, Austria

2 Acoustics Research Institute, Austrian Academy of Sciences, Wohllebengasse 12-14, 1040 Vienna, Austria

3 Delft University of Technology, Mekelweg 4, Building 36, 2628 CD Delft, The Netherlands

4 Lehrstuhl Reliable Machine Learning, Katholische Universität Eichstätt-Ingolstadt, Ostenstrasse 26, 85072 Eichstätt, Germany
} 


\section{Introduction}

Given a countable collection $\left(g_{j}\right)_{j \in J}$ of functions $g_{j}: \mathbb{R}^{d} \rightarrow \mathbb{C}$ and a collection $\left(C_{j}\right)_{j \in J}$ of matrices $C_{j} \in \mathrm{GL}(d, \mathbb{R})$, we consider the structured function system

$$
\left(T_{\gamma} g_{j}\right)_{j \in J, \gamma \in C_{j} \mathbb{Z}^{d}}=\left(g_{j}(\cdot-\gamma)\right)_{j \in J, \gamma \in C_{j} \mathbb{Z}^{d}},
$$

and aim to represent a function or distribution $f$ as a linear combination

$$
f=\sum_{j \in J} \sum_{\gamma \in C_{j} \mathbb{Z}^{d}} c_{j, \gamma} T_{\gamma} g_{j}
$$

In many important examples of this formalism, the functions $g_{j}$ are obtained through affine transforms (in the Fourier domain) of a single function $g$. For instance, in dimension $d=1$, the well-known wavelet [19] and Gabor systems [34] are obtained as

$$
\begin{array}{lll}
g_{j}(x):=2^{j / 2} g\left(2^{j} x\right), & j \in \mathbb{Z}, & C_{j}=2^{j}, \\
g_{j}(x):=e^{2 \pi i j x} g(x), & j \in \beta \mathbb{Z}, & C_{j}=\alpha .
\end{array}
$$

For $d>1$, anisotropic wavelet systems provide additional important examples, see e.g., $[2,12,47]$.

We are interested in the ability of (1.1) to reproduce all functions or distributions $f$ in various function spaces by a suitably convergent series (1.2). For the Hilbert space $L^{2}\left(\mathbb{R}^{d}\right)$ this task is significantly easier: it amounts to establishing the frame inequalities

$$
\|f\|_{L^{2}}^{2} \asymp \sum_{j \in J} \sum_{\gamma \in C_{j} \mathbb{Z}^{d}} \|\left. f\left|T_{\gamma} g_{j}\right\rangle\right|^{2} \quad \forall f \in L^{2}\left(\mathbb{R}^{d}\right) .
$$

Indeed, the norm equivalence (1.5) means that the frame operator $S: L^{2}\left(\mathbb{R}^{d}\right) \rightarrow$ $L^{2}\left(\mathbb{R}^{d}\right)$,

$$
S f:=\sum_{j \in J} \sum_{\gamma \in C_{j} \mathbb{Z}^{d}}\left\langle f \mid T_{\gamma} g_{j}\right\rangle T_{\gamma} g_{j}
$$

is bounded and invertible on $L^{2}\left(\mathbb{R}^{d}\right)$, and consequently (1.2) holds with $c_{j, \gamma}=$ $\left\langle S^{-1} f \mid T_{\gamma} g_{j}\right\rangle$.

The validity of the frame inequalities is closely related to the covering properties of the Fourier transforms of the generating functions $\widehat{g_{j}}$, which is encoded in the Calderón condition:

$$
\sum_{j \in J} \frac{1}{\left|\operatorname{det} C_{j}\right|}\left|\widehat{g_{j}}\right|^{2} \asymp 1, \quad \text { a.e. }
$$


This connection is most apparent in the so-called painless case, in which the supports of the functions $\widehat{g_{j}}$ are compact. Under this assumption, the expansion (1.2) is a local Fourier expansion

$$
\widehat{f}(\xi)=\sum_{j \in J} \sum_{\gamma \in C_{j} \mathbb{Z}^{d}} c_{j, \gamma} e^{-2 \pi i \gamma \xi} \widehat{g_{j}}(\xi) .
$$

In many important cases, the functions $g_{j}$ are not bandlimited, but have a well concentrated frequency profile, such as a Gaussian. Then (1.7) is an almost-local Fourier expansion, that one still expects to be governed by (1.6) —and, indeed, under mild conditions, (1.6) is necessary for (1.5) to hold [18, 30].

The formal analysis of non-painless expansions with a reproducing system (1.1) relies on a remarkable representation of the frame operator in the Fourier domain, namely

$$
\widehat{S f}(\xi)=\sum_{\alpha \in \Lambda} t_{\alpha}(\xi-\alpha) \widehat{f}(\xi-\alpha),
$$

where $t_{\alpha}(\xi)=\sum_{j \in \kappa(\alpha)} \frac{1}{\left|\operatorname{det} C_{j}\right|} \widehat{\widehat{g}_{j}(\xi)} \widehat{g_{j}}(\xi+\alpha)$; here, the translation nodes $\Lambda \subseteq$ $\mathbb{R}^{d}$ and indices $\kappa(\alpha) \subseteq J$ are determined by the matrices $C_{j}$ (see (5.2) below). For Gabor expansions, the representation (1.8) is known under the name of Walnut's representation [63] while for wavelets it is attributed to Daubechies and Tchamitchian [19,Chapter 3]. The theory of generalized shift-invariant systems [39, 53] establishes the general form of (1.8) and exploits its many consequences. For example, tight frames - that is, systems for which equality holds in (1.5) — are characterized by a set of algebraic relations involving the functions $t_{\alpha}$; see [39].

\subsection{The Walnut-Daubechies Criterion}

The multiplier $t_{0}$ associated with $\alpha=0$ in (1.8) is precisely the Calderón sum appearing in (1.6); that is,

$$
t_{0}(\xi)=\sum_{j \in J} \frac{1}{\left|\operatorname{det} C_{j}\right|}\left|\widehat{g_{j}}(\xi)\right|^{2}
$$

A powerful frame criterion arises by comparing the representation of $S$ given in (1.8) to the diagonal term $\mathcal{F}^{-1}\left(t_{0} \cdot \widehat{f}\right)$, and by estimating the corresponding discrepancy. In the model cases of Gabor and wavelets systems, these criteria are again attached to the names of Walnut and Daubechies, and are particularly useful for studying Gaussian wave-packets, which have fast-decaying frequency tails, but do not yield tight frames. A general version of the Walnut-Daubechies criterion also holds for generalized shiftinvariant systems under mild assumptions $[17,45]$; this criterion is greatly useful in the construction of anisotropic time-scale decompositions-see e.g. [20].

The price to pay for the flexibility of the Walnut-Daubechies criterion is that it does not produce an explicit dual system implementing the coefficient functionals 
$f \mapsto c_{j, \gamma}$ in (1.2). Rather, it only yields an $L^{2}$ norm estimate which is sufficient to establish (1.5) but does not imply the convergence of (1.8) in other norms. In contrast, explicit constructions of frame pairs, that is, frames where the coefficient functionals are given by

$$
c_{j, \gamma}=\left\langle f \mid T_{\gamma} h_{j}\right\rangle
$$

for another reproducing system $\left\{h_{j}: j \in J\right\}$, naturally extend to many other Banach spaces besides $L^{2}\left(\mathbb{R}^{d}\right)$. These spaces are determined by the concentration of the Fourier support of the generators $g_{j}$, and are generically called Besov-type spaces [56,Chapter 2] [58]. The model case is given by (1.3), where the functions $\widehat{g_{j}}$ form a so-called Littlewood-Paley decomposition.

The goal of this article is to derive a variant of the Walnut-Daubechies criterion which implies that the frame operator is invertible in such Besov-type spaces.

\subsection{Besov-Type Decomposition Spaces}

For the informal definition of Besov-type spaces, fix a cover $\mathcal{Q}=\left(Q_{i}\right)_{i \in I}$ of a full measure open subset in the Fourier domain $\widehat{\mathbb{R}}^{d}$. We impose a mild admissibility condition by limiting the number of overlaps between different elements of $\mathcal{Q}$ - see Section 3 for the precise condition. Given a suitable partition of unity $\left(\varphi_{i}\right)_{i \in I}$ subordinate to $\mathcal{Q}$, together with a suitable (so-called $\mathcal{Q}$-moderate) weight function $w: I \rightarrow(0, \infty)$, the space $\mathcal{D}\left(\mathcal{Q}, L^{p}, \ell_{w}^{q}\right)$, for $p, q \in[1, \infty]$, is defined as the space of distributions $f$ satisfying

$$
\|f\|_{\mathcal{D}\left(\mathcal{Q}, L^{p}, \ell_{w}^{q}\right)}:=\left\|\left(\left\|\mathcal{F}^{-1}\left(\varphi_{i} \cdot \widehat{f}\right)\right\|_{L^{p}}\right)_{i \in I}\right\|_{\ell_{w}^{q}}=\left\|\left(w_{i} \cdot\left\|\mathcal{F}^{-1}\left(\varphi_{i} \cdot \widehat{f}\right)\right\|_{L^{p}}\right)_{i \in I}\right\|_{\ell q}<\infty,
$$

where $\mathcal{F}^{-1}$ denotes the inverse Fourier transform. Provided that an adequate notion of distribution is used in the definitions, the spaces $\mathcal{D}\left(\mathcal{Q}, L^{p}, \ell_{w}^{q}\right)$ form Banach spaces and are independent of the particular (sufficiently regular) partition of unity used to define them.

The construction of Besov-type spaces follows the so-called decomposition method [56,Chapter 2], [58,Section 1.2], yielding an instance of the so-called spaces defined by decomposition methods [55], or decomposition spaces [23, 57] in more abstract settings. This is why we also use the term Besov-type decomposition spaces. Uniform Besov-type spaces, associated with the cover $\mathcal{Q}$ consisting of integer translates of a cube, are known as modulation spaces [22], while a dyadic frequency cover yields the usual Besov spaces [27, 49] —-see also [56,Section 2.2]. When the cover is generated by powers of an expansive matrix, one obtains anisotropic Besov spaces $[8,12,13,56]$. We remark that the range of spaces defined by (1.9) does not include Triebel-Lizorkin spaces [28]. 


\subsection{Overview of the Results}

We state a simplified version of our main results for systems of the form (1.1) with generating functions $g_{j} \in L^{1}\left(\mathbb{R}^{d}\right) \cap L^{2}\left(\mathbb{R}^{d}\right)$ with $\widehat{g} \in C^{\infty}\left(\widehat{\mathbb{R}}^{d}\right)$, given by

$$
g_{j}=\left|\operatorname{det} A_{j}\right|^{-1 / 2} \cdot \mathcal{F}^{-1}\left(\widehat{g} \circ S_{j}^{-1}\right)=\left|\operatorname{det} A_{j}\right|^{1 / 2} \cdot e^{2 \pi i\left\langle b_{j}, \cdot\right\rangle} \cdot\left(g \circ A_{j}^{t}\right),
$$

for (invertible) affine maps $S_{j}=A_{j}(\cdot)+b_{j}$ and translation matrices $C_{j}=\delta A_{j}^{-t}$ with $\delta>0$. The parameter $\delta>0$ is a resolution parameter that controls the density of the translation nodes in (1.1).

To define Besov-type spaces adapted to the frequency concentration of the system $\left(g_{j}\right)_{j \in J}$, we also consider an affinely generated cover $\mathcal{Q}=\left(Q_{j}\right)_{j \in J}$ of the form $Q_{j}=A_{j} Q+b_{j}$. If $\widehat{g}$ is mostly concentrated inside the basic set $Q$, then (1.10) implies that $\widehat{g_{j}}$ is localized around $Q_{j}$. Under these assumptions, the Calderón condition reads

$$
0<\mathrm{A} \leq \sum_{j \in J}\left|\widehat{g}\left(S_{j}^{-1} \xi\right)\right|^{2} \leq \mathrm{B}<\infty, \quad \text { a.e. }
$$

which means that $\left(\widehat{g_{j}}\right)_{j \in J}$ is approximately a partition of unity adapted to $\mathcal{Q}$.

The following is our main result, proved in Section 7.3.

Theorem 1.1 For each affinely generated cover $\mathcal{Q}=\left(A_{j} Q+b_{j}\right)_{j \in J}=\left(S_{j} Q\right)_{j \in J}$ of an open, co-null set $\mathcal{O} \subset \widehat{\mathbb{R}}^{d}$, and each $\mathcal{Q}$-moderate weight $w=\left(w_{j}\right)_{j \in J}$, there exists a constant $C_{d, \mathcal{Q}, w}$ with the following property: Suppose that $\left(g_{j}\right)_{j \in J}$ is compatible with $\mathcal{Q}$ in the sense of (1.10) and that the Calderón condition (1.11) holds. Moreover, suppose that

$$
M_{0}:=\sup _{i \in J} \sum_{j \in J} \max \left\{1,\left\|A_{j}^{-1} A_{i}\right\|^{d+1}\right\}\left(\int_{Q} \max _{|\alpha| \leq d+1}\left|\left(\partial^{\alpha} \widehat{g}\right)\left(S_{j}^{-1}\left(S_{i} \xi\right)\right)\right|^{2(d+1)} d \xi\right)^{\frac{1}{d+1}}<\infty
$$

and that $M_{1}:=\max \left\{\sup _{i \in J} \sum_{j \in J} M_{i, j}, \sup _{j \in J} \sum_{i \in J} M_{i, j}\right\}<\infty$, where

$$
M_{i, j}:=L_{i, j} \cdot \int_{Q}\left(1+\left|S_{j}^{-1}\left(S_{i} \xi\right)\right|\right)^{2 d+2} \max _{|\alpha| \leq d+1}\left|\left(\partial^{\alpha} \widehat{g}\right)\left(S_{j}^{-1}\left(S_{i} \xi\right)\right)\right| d \xi
$$

and $L_{i, j}:=\max \left\{\frac{w_{i}}{w_{j}}, \frac{w_{j}}{w_{i}}\right\} \cdot\left(\max \left\{1,\left\|A_{i}^{-1} A_{j}\right\|^{2}\right\} \max \left\{1,\left\|A_{j}^{-1} A_{i}\right\|^{3}\right\}\right)^{d+1}$ for $i, j \in$ J. Choose $\delta>0$ such that

$$
C_{d, \mathcal{Q}, w} M_{0}^{\frac{d+1}{d+2}} M_{1}^{\frac{2}{d+2}} \delta<\mathrm{A}
$$

Then the frame operator associated to $\left(T_{\delta A_{j}^{-t} k} g_{j}\right)_{j \in J, k \in \mathbb{Z}^{d}}$ is well-defined, bounded, and invertible on $\mathcal{D}\left(\mathcal{Q}, L^{p}, \ell_{w}^{q}\right)$ for all $p, q \in[1, \infty]$. The value of the constant $C_{d, \mathcal{Q}, w}$ is given in Theorem 7.5 below. 
The quantities $M_{0}$ and $M_{1}$ in Theorem 1.1 control the interaction between the generators $g_{j}$ and the elements of the cover $\mathcal{Q}$. In contrast to the classical $L^{2}$ WalnutDaubechies criterion, the derivatives of $\widehat{g}$ are now involved. We also prove a more technical version of Theorem 1.1 in which the generators need not exactly be affine images (in the Fourier domain) of a single function, but only approximately so. This is important, for example, to describe non-homogeneous time-scale systems, which contain a low-pass and a high-pass window. We refer the reader to [62] for a detailed discussion of concrete examples and calculations that can be used also in our framework.

Although the constant $C_{d, \mathcal{Q}, w}$ in Theorem 1.1 is explicit, it is too large to be used as a guide for concrete numerical implementations. We also derive a version of the criterion with more favorable constants, but which only provides expansions on $L^{2}$ based Besov-type spaces; see Sect. 5.5.

A result closely related to Theorem 1.1 was recently obtained by the third named author in [62] — see the discussion below. While our techniques are significantly different from those in [62] — and, indeed, we regard the simplicity of the present methods a main contribution - we remark that we make use of several auxiliary results obtained in [62].

Under the conditions of Theorem 1.1, the coefficient and reconstruction operators

$$
\mathscr{C}: f \mapsto\left(\left\langle f\left|T_{\gamma} g_{j}\right|\right)_{j \in J, \gamma \in C_{j} \mathbb{Z}^{d}} \text { and } \mathscr{D}: c=\left(c_{j, \gamma}\right)_{j \in J, \gamma \in C_{j} \mathbb{Z}^{d}} \mapsto \sum_{j \in J} \sum_{\gamma \in C_{j} \mathbb{Z}^{d}} c_{j, \gamma} T_{\gamma} g_{j}\right.
$$

define bounded operators between the Besov-type space $\mathcal{D}\left(\mathcal{Q}, L^{p}, \ell_{w}^{q}\right)$ and suitable sequence spaces (see Sect. 4). As a consequence, the invertibility of the frame operator on the spaces $\mathcal{D}\left(\mathcal{Q}, L^{p}, \ell_{w}^{q}\right)$ implies that the $L^{2}$-convergent canonical frame expansions

$$
f=\sum_{j \in J} \sum_{\gamma \in C_{j} \mathbb{Z}^{d}}\left\langle S^{-1} f\left|T_{\gamma} g_{j}\right| T_{\gamma} g_{j}=\sum_{j \in J} \sum_{\gamma \in C_{j} \mathbb{Z}^{d}}\left\langle f\left|T_{\gamma} g_{j}\right| S^{-1} T_{\gamma} g_{j}\right.\right.
$$

extend to series convergent in Besov-type norms (or weak- $*$-convergent for $p=$ $\infty$ or $q=\infty$ ). In more technical terms, the canonical Hilbert-space dual frame $\left\{S^{-1} T_{\gamma} g_{j}: j \in J, \gamma \in C_{j} \mathbb{Z}^{d}\right\}$ provides a Banach frame and an atomic decomposition for the Besov-type spaces $\mathcal{D}\left(\mathcal{Q}, L^{p}, \ell_{w}^{q}\right)$. This is a novel feature of Theorem 1.1: other results on the existence of series expansions, based on so-called oscillation estimates, show that the coefficient and reconstruction maps (1.12) are respectively left and right invertible on the Besov-type spaces, but do not yield consequences for the Hilbert space pseudo-inverses $\mathscr{C}^{\dagger}=S^{-1} \mathscr{D}$ and $\mathscr{D}^{\dagger}=\mathscr{C} S^{-1}[24,33,62]$. In contrast, Theorem 1.1 concerns $\mathscr{C}^{\dagger}, \mathscr{D}^{\dagger}$ - see Corollary 7.6-and implies that operations on the canonical frame expansions (1.13) that decrease the magnitude of the coefficients, such as thresholding, are uniformly bounded in Besov-type norms. More precisely, if for each $j \in J$ and $\gamma \in C_{j} \mathbb{Z}^{d}$, we are given a function $\Phi_{j, \gamma}: \mathbb{C} \rightarrow \mathbb{C}$ satisfying 
$\left|\Phi_{j, \gamma}(x)\right| \leq C|x|$, then the maps

$$
f \mapsto \sum_{j \in J} \sum_{\gamma \in C_{j} \mathbb{Z}^{d}} \Phi_{j, \gamma}\left(\left\langle S^{-1} f \mid T_{\gamma} g_{j}\right\rangle\right) T_{\gamma} g_{j}
$$

and

$$
f \mapsto \sum_{j \in J} \sum_{\gamma \in C_{j} \mathbb{Z}^{d}} \Phi_{j, \gamma}\left(\left\langle f \mid T_{\gamma} g_{j}\right\rangle\right) S^{-1} T_{\gamma} g_{j}
$$

are bounded (possibly non-linear) operators on all of the spaces $\mathcal{D}\left(\mathcal{Q}, L^{p}, \ell_{w}^{q}\right)$. In particular, frame multipliers with bounded symbols—see e.g. [7]—define bounded operators on Besov-type spaces.

\subsection{Related Work}

The theory of localized frames. The uniform frequency cover $\left\{(-1,1)^{d}+k: k \in \mathbb{Z}^{d}\right\}-$ which gives rise to Gabor systems (1.4) — is special in that every reproducing system (1.1) satisfying the frame inequalities (1.5), and mild smoothness and decay conditions, provides also expansions for other Banach spaces (the precise range of spaces being determined by the particular smoothness and decay of the generators). Indeed, the theory of localized frames $[4,5,35]$ implies that the frame operator is invertible on modulation spaces. Similar results hold for $L^{p}$ spaces [6, 43]. Thus, in these cases, the classical Walnut-Daubechies criterion has consequences for Banach spaces besides $L^{2}$ — without having to adjust the density $\delta$ - and Theorem 1.1 does not add anything interesting.

The key tool of the theory of localized frames is the spectral invariance of certain matrix algebras. Such tools are not applicable to general admissible covers as considered in this article. Indeed, it is known that the frame operator associated with certain smooth and fast-decaying wavelets with several vanishing moments fails to be invertible on $L^{p}$-spaces [46, Chapter 4]. In connection to this point, we mention that the Mexican hat wavelet satisfies Daubechies criterion, but the validity of the corresponding $L^{p}$ expansions was established only recently with significant ad-hoc work [15].

Almost painless generators and homogeneous covers. There is a well-developed literature related to the so-called painless expansions on decomposition spaces. The first construction of Banach frames for general decomposition spaces was given by Borup and Nielsen [11] using bandlimited generators. This construction was then complemented with a delicate perturbation argument to produce compactly supported frames [48]—see also [16, 44]. The constructions in [48] for Besov-type spaces are restricted to so-called homogeneous covers, which are generated by applying integer powers of a matrix to a given set. This restriction rules out some important examples such as inhomogeneous dyadic covers and many popular wavepacket systems. 
Invertibility of the frame operator versus existence of left and right inverses. The first construction of time-scale decompositions proceeded by discretizing Calderón's reproducing formula through Riemann-like sums [29]. A similar approach works for the voice transform associated with any integrable unitary representation and is the basis of the so-called coorbit theory [24]. To some extent, those techniques extend to any integral transform, provided that one can control its modulus of continuity [38]. Such an approach was used by the third named author to construct compactly supported Banach frames and atomic decompositions in Besov-type spaces [62]. The main result of [62] is qualitatively similar to Theorem 1.1, but only concludes the existence of left and right inverses for the coefficient and synthesis maps, acting on respective Banach spaces. In contrast, we show that the Hilbert space frame operator is simultaneously invertible on all the relevant Banach spaces. The advantage of the present approach is that we are able to show that the Hilbert spaces series-which are defined by minimizing the $\ell^{2}$ norm of the coefficients in (1.2) - extend to series convergent in Besov-type spaces, and thus many operations on the canonical frame expansion are also shown to be bounded in Besov-type spaces. On the other hand, there are situations in which there exists a left inverse for the coefficient operator (or a right inverse for the reconstruction operator), but the frame operator is not invertible. For example, a wavelet system generated by a smooth mother wavelet without vanishing moments can generate an atomic decomposition for the Besov spaces $B_{p, q}^{s}\left(\mathbb{R}^{d}\right)$ of strictly positive smoothness $s>0$ without yielding a frame [62,Proposition 8.4]. Such examples are not covered by our results.

Quasi-Banach spaces. We do not treat the quasi-Banach range $p, q \in(0, \infty]$, which is treated in [62]. We expect the tools developed in [62] for treating the quasi-Banach range to be also applicable to the present setting, and to yield an extension of our main results to the quasi-Banach range.

\subsection{Technical Overview and Organization}

Our approach is as follows: we consider the Walnut-Daubechies representation (1.8) of the frame operator and bound the discrepancy between $S f$ and the diagonal term $\mathcal{F}^{-1}\left(t_{0} \cdot \widehat{f}\right)$ in a Besov-type norm. To this end, we estimate each Fourier multiplier $t_{\alpha}$ with a Sobolev embedding, and control the inverse Fourier multiplier $1 / t_{0}$ by directly bounding the terms in Faà di Bruno's formula.

The main estimates are derived in decreasing level of generality. We first consider very general covers $\mathcal{Q}=\left(Q_{i}\right)_{i \in I}$ and an abstract notion of molecule, which models the interaction between the generators $g_{j}$ of the system $\left(T_{\gamma} g_{j}\right)_{j \in J, \gamma \in C_{j} \mathbb{Z}^{d}}$ and the elements $Q_{i}$ of the cover $\mathcal{Q}$. Here, the associated index sets $I$ and $J$ do not need to coincide. We then provide simplified estimates for affinely generated covers. The limiting cases $p, q=\infty$ involve delicate approximation arguments that may be of independent interest.

The paper is organized as follows: Sect. 2 introduces notation and preliminaries. Besov-type spaces are introduced in Sect. 3. Section 4 treats the boundedness of the coefficient, synthesis and frame operators on suitable spaces. Section 5 is concerned with the invertibility of the frame operator and provides estimates for the abstract 
Walnut-Daubechies criterion. These estimates are further simplified in Sects. 6 and 7 for affinely generated covers and suitably adapted generating functions. Several technical results are deferred to the appendices.

\section{Notation and Preliminaries}

\subsection{General Notation}

We let $\mathbb{N}:=\{1,2,3, \ldots\}$, and $\mathbb{N}_{0}:=\mathbb{N} \cup\{0\}$. For $n \in \mathbb{N}_{0}$, we write $\underline{n}:=\{1, \ldots, n\}$; in particular, $\underline{0}=\emptyset$. For a multi-index $\beta \in \mathbb{N}_{0}^{d}$, its length is $|\beta|=\sum_{i=1}^{d}\left|\beta_{i}\right|$.

The conjugate exponent $p^{\prime}$ of $p \in(1, \infty)$ is defined as $p^{\prime}:=\frac{p}{p-1}$. We let $1^{\prime}:=\infty$ and $\infty^{\prime}:=1$.

Given two functions $f, g: X \rightarrow[0, \infty)$, we write $f \lesssim g$ provided that there exists a constant $C>0$ such that $f(x) \leq C g(x)$ for all $x \in X$. We write $f \asymp g$ for $f \lesssim g$ and $g \lesssim f$.

The dot product of $x, y \in \mathbb{R}^{d}$ is written $x \cdot y:=\sum_{i=1}^{d} x_{i} y_{i}$. The Euclidean norm of a vector $x \in \mathbb{R}^{d}$ is denoted by $|x|:=\sqrt{x \cdot x}$. The open Euclidean ball, with radius $r>0$ and center $x \in \mathbb{R}^{d}$, is denoted by $B_{r}(x)$, and the corresponding closed ball is denoted by $\overline{B_{r}}(x)$. More generally, the closure of a set $M \subseteq \mathbb{R}^{d}$ is denoted by $\bar{M}$.

The cardinality of a set $X$ will be denoted by $|X| \in \mathbb{N}_{0} \cup\{\infty\}$. The Lebesgue measure of a Borel measurable set $E \subset \mathbb{R}^{d}$ will be denoted by $\lambda(E)$. Given a subset $M \subset X$, we define its indicator function $\mathbb{1}_{M}: X \rightarrow\{0,1\}$ by requiring $\mathbb{1}_{M}(x)=1$ if $x \in M$ and $\mathbb{1}_{M}(x)=0$ otherwise.

For a matrix $M \in \mathbb{C}^{I \times J}$, its Schur norm is defined as

$$
\|M\|_{\text {Schur }}:=\max \left\{\sup _{i \in I} \sum_{j \in J}\left|M_{i, j}\right|, \sup _{j \in J} \sum_{i \in I}\left|M_{i, j}\right|\right\} \in[0, \infty] .
$$

A matrix $M \in \mathbb{C}^{I \times J}$ satisfying $\|M\|_{\text {Schur }}<\infty$ is said to be of Schur-type. A Schur-type matrix $M \in \mathbb{C}^{I \times J}$ induces a bounded linear operator $\mathbf{M}: \ell^{p}(J) \rightarrow$ $\ell^{p}(I), \quad\left(c_{j}\right)_{j \in J} \mapsto\left(\sum_{j \in J} M_{i, j} c_{j}\right)_{i \in I}$, with $\|\mathbf{M}\|_{\ell^{p} \rightarrow \ell^{p}} \leq\|M\|_{\text {Schur }}$ for all $p \in$ $[1, \infty]$; this is called Schur's test. For a proof of a (weighted) version of Schur's test, cf. [37,Lemma 4].

\subsection{Fourier Analysis}

The translate of $f: \mathbb{R}^{d} \rightarrow \mathbb{C}$ by $y \in \mathbb{R}^{d}$ is denoted by $T_{y} f(x)=f(x-y)$. We denote by $\widehat{\mathbb{R}}^{d}$ the Fourier domain of $\mathbb{R}^{d}$. Modulation of $f: \mathbb{R}^{d} \rightarrow \mathbb{C}$ by $\xi \in \widehat{\mathbb{R}}^{d}$ is denoted by $M_{\xi} f(x):=e^{2 \pi i \xi \cdot x} f(x)$. The Fourier transform $\mathcal{F}: L^{1}\left(\mathbb{R}^{d}\right) \rightarrow C_{0}\left(\widehat{\mathbb{R}}^{d}\right), f \mapsto \widehat{f}$ is normalized as

$$
\widehat{f}(\xi)=\int_{\mathbb{R}^{d}} f(x) e^{-2 \pi i x \cdot \xi} d x
$$


for $\xi \in \widehat{\mathbb{R}}^{d}$. Similarly normalized, we define $\mathcal{F}: L^{1}\left(\widehat{\mathbb{R}}^{d}\right) \rightarrow C_{0}\left(\mathbb{R}^{d}\right)$. The inverse Fourier transform $\mathcal{F}^{-1} f:=\widehat{f}(-\cdot) \in C_{0}\left(\mathbb{R}^{d}\right)$ of $f \in L^{1}\left(\widehat{\mathbb{R}}^{d}\right)$ will occasionally also be denoted by $\breve{f}$. Similar notation will be used for the (unitary) Fourier-Plancherel transform $\mathcal{F}: L^{2}\left(\mathbb{R}^{d}\right) \rightarrow L^{2}\left(\widehat{\mathbb{R}}^{d}\right)$.

The test space of compactly supported, smooth functions on an open set $\mathcal{O} \subset \mathbb{R}^{d}$ will be denoted by $C_{c}^{\infty}(\mathcal{O})$. The topology on $C_{c}^{\infty}(\mathcal{O})$ is taken to be the usual topology defined through the inductive limit of Fréchet spaces; see [54,Sect. 6.2] for the details. The sesquilinear dual pairing between $\mathcal{D}(\mathcal{O}):=C_{c}^{\infty}(\mathcal{O})$ and its dual $\mathcal{D}^{\prime}(\mathcal{O})$ is given by $\langle f \mid g\rangle_{\mathcal{D}^{\prime}, \mathcal{D}}:=f(\bar{g})$ for $f \in \mathcal{D}^{\prime}(\mathcal{O})$ and $g \in C_{c}^{\infty}(\mathcal{O})$.

The Schwartz space is denoted by $\mathcal{S}\left(\mathbb{R}^{d}\right)$ and its topological dual will be denoted by $\mathcal{S}^{\prime}\left(\mathbb{R}^{d}\right)$. The canonical extension of the Fourier transform to $\mathcal{S}^{\prime}\left(\mathbb{R}^{d}\right)$ is denoted by $\mathcal{F}: \mathcal{S}^{\prime}\left(\mathbb{R}^{d}\right) \rightarrow \mathcal{S}^{\prime}\left(\widehat{\mathbb{R}}^{d}\right)$, that is, $\langle\mathcal{F} f, g\rangle_{\mathcal{S}^{\prime}, \mathcal{S}}=\langle f, \mathcal{F} g\rangle_{\mathcal{S}^{\prime}, \mathcal{S}}$ for $f \in \mathcal{S}^{\prime}\left(\mathbb{R}^{d}\right)$ and $g \in \mathcal{S}\left(\widehat{\mathbb{R}}^{d}\right)$. We denote bilinear dual pairings by $\langle\cdot, \cdot\rangle$, while $\langle\cdot \mid \cdot\rangle$ denotes a sesquilinear dual pairing, which is anti-linear in the second component.

Lastly, for $p \in[1, \infty]$ we define $\mathcal{F} L^{p}\left(\mathbb{R}^{d}\right):=\left\{\widehat{f}: f \in L^{p}\left(\mathbb{R}^{d}\right)\right\} \subset \mathcal{S}^{\prime}\left(\widehat{\mathbb{R}}^{d}\right)$, equipped with the norm $\|f\|_{\mathcal{F}_{L^{p}}}:=\left\|\mathcal{F}^{-1} f\right\|_{L^{p}}$. Here, note that $\|f \cdot g\|_{\mathcal{F}_{L^{p}}} \leq$ $\|f\|_{\mathcal{F} L^{1}} \cdot\|g\|_{\mathcal{F} L^{p}}$, where the exact nature of the product $f \cdot g$ is explained in more detail in Definition 5.5. Furthermore, for any invertible affine-linear map $S: \widehat{\mathbb{R}}^{d} \rightarrow \widehat{\mathbb{R}}^{d}$, one has $\|f \circ S\|_{\mathcal{F} L^{1}}=\|f\|_{\mathcal{F} L^{1}}$.

\subsection{Amalgam Spaces}

Let $U \subset \mathbb{R}^{d}$ be a bounded Borel set with non-empty interior. The Amalgam space $W_{U}\left(L^{\infty}, L^{1}\right)$ is the space of all $f \in L^{\infty}\left(\mathbb{R}^{d}\right)$ satisfying

$$
\|f\|_{W_{U}\left(L^{\infty}, L^{1}\right)}:=\int_{\mathbb{R}^{d}}\|f\|_{L^{\infty}(U+x)} d x<\infty .
$$

The (closed) subspace of $W_{U}\left(L^{\infty}, L^{1}\right)$ consisting of continuous functions is denoted $W_{U}\left(C_{0}, L^{1}\right)$.

The space $W\left(L^{\infty}, L^{1}\right):=W_{U}\left(L^{\infty}, L^{1}\right)$ is independent of the choice of $U$, with equivalent norms for different choices. In particular, if $A \in \mathrm{GL}\left(\mathbb{R}^{d}\right)$, then

$$
\|f\|_{W_{A U}\left(L^{\infty}, L^{1}\right)}=|\operatorname{det} A| \cdot\|f \circ A\|_{W_{U}\left(L^{\infty}, L^{1}\right)},
$$

an identity that will be used repeatedly. It is readily seen that the space $W_{U}\left(L^{\infty}, L^{1}\right)$ is an $L^{1}$-convolution module; that is, if $f \in L^{1}\left(\mathbb{R}^{d}\right)$ and $g \in W_{U}\left(L^{\infty}, L^{1}\right)$, then the product $f * g \in W_{U}\left(L^{\infty}, L^{1}\right)$, with $\|f * g\|_{W_{U}\left(L^{\infty}, L^{1}\right)} \leq\|f\|_{L^{1}}\|g\|_{W_{U}\left(L^{\infty}, L^{1}\right)}$, simply because of $\|f * g\|_{L^{\infty}(U+x)} \leq\left(|f| *\left[y \mapsto\|g\|_{L^{\infty}(U+y)}\right]\right)(x)$.

Lastly, there is an equivalent discrete norm on $W\left(L^{\infty}, L^{1}\right)$, namely

$$
\|f\|_{W\left(L^{\infty}, \ell^{1}\right)}:=\sum_{n \in \mathbb{Z}^{d}}\left\|\mathbb{1}_{n+[0,1]^{d}} \cdot f\right\|_{L^{\infty}} .
$$


The global component in this norm is denoted by $\ell^{1}$ rather than $L^{1}$ to distinguish it from $\|\cdot\|_{W_{U}\left(L^{\infty}, L^{1}\right)}$. The norm $\|\cdot\|_{W\left(C_{0}, \ell^{1}\right)}$ is simply the restriction of $\|\cdot\|_{W\left(L^{\infty}, \ell^{1}\right)}$ to $W_{U}\left(C_{0}, L^{1}\right)$.

The reader is referred to [26, 40] for background on amalgam spaces and to [21] for a far-reaching generalization that includes the combination of smoothness and decay conditions.

\section{Besov-Type Spaces}

This section introduces decomposition spaces, and related notions such as covers, weights and bounded admissible partitions of unity (BAPUs).

\subsection{Covers and BAPUs}

Definition 3.1 Let $\mathcal{O} \neq \emptyset$ be an open subset of $\widehat{\mathbb{R}}^{d}$. A family $\mathcal{Q}=\left(Q_{i}\right)_{i \in I}$ of subsets $Q_{i} \subset \mathcal{O}$ is called an admissible cover of $\mathcal{O}$ if

(i) $\mathcal{Q}$ is a cover of $\mathcal{O}$, that is, $\mathcal{O}=\bigcup_{i \in I} Q_{i}$;

(ii) $Q_{i} \neq \emptyset$ for all $i \in I$;

(iii) $N_{\mathcal{Q}}:=\sup _{i \in I}\left|i^{*}\right|<\infty$, where $i^{*}:=\left\{\ell \in I: Q_{\ell} \cap Q_{i} \neq \emptyset\right\}$ for $i \in I$.

A sequence $w=\left(w_{i}\right)_{i \in I}$ in $(0, \infty)$ is called a $\mathcal{Q}$-moderate weight if

$$
C_{w, \mathcal{Q}}:=\sup _{i \in I} \sup _{\ell \in i^{*}} \frac{w_{i}}{w_{\ell}}<\infty .
$$

For a weight $w=\left(w_{i}\right)_{i \in I}$ in $(0, \infty)$ and an exponent $q \in[1, \infty]$, we define

$$
\ell_{w}^{q}(I):=\left\{c=\left(c_{i}\right)_{i \in I} \in \mathbb{C}^{I}:\|c\|_{\ell_{w}^{q}}:=\left\|\left(w_{i} \cdot c_{i}\right)_{i \in I}\right\|_{\ell^{q}}<\infty\right\} .
$$

The significance of a $\mathcal{Q}$-moderate weight is that the associated $\mathcal{Q}$-clustering map is well-defined and bounded. The precise statement is as follows; see [61,Lemma 4.13].

Lemma 3.2 Let $q \in[1, \infty]$. Suppose that $\mathcal{Q}=\left(Q_{i}\right)_{i \in I}$ is an admissible cover of an open subset $\mathcal{O} \subset \widehat{\mathbb{R}}^{d}$ and that the weight $w=\left(w_{i}\right)_{i \in I}$ is $\mathcal{Q}$-moderate. Then the $\mathcal{Q}$-clustering map

$$
\Gamma_{\mathcal{Q}}: \ell_{w}^{q}(I) \rightarrow \ell_{w}^{q}(I), \quad\left(c_{i}\right)_{i \in I} \mapsto\left(c_{i}^{*}\right)_{i \in I},
$$

where $c_{i}^{*}:=\sum_{\ell \in i^{*}} c_{\ell}$, is well-defined and bounded, with $\left\|\Gamma_{\mathcal{Q}}\right\|_{\ell_{w}^{q} \rightarrow \ell_{w}^{q}} \leq C_{w, \mathcal{Q}} \cdot N_{\mathcal{Q}}$.

The next definition clarifies our assumptions regarding the partitions of unity that are suitable for defining the decomposition space norm.

Definition 3.3 Let $\mathcal{Q}=\left(Q_{i}\right)_{i \in I}$ be an admissible cover of an open subset $\emptyset \neq \mathcal{O} \subset$ $\widehat{\mathbb{R}}^{d}$. A family $\Phi=\left(\varphi_{i}\right)_{i \in I}$ is called a bounded admissible partition of unity (BAPU), subordinate to $\mathcal{Q}$, if 
(i) $\varphi_{i} \in C_{c}^{\infty}(\mathcal{O}) \subset \mathcal{S}\left(\widehat{\mathbb{R}}^{d}\right)$ for all $i \in I$;

(ii) $\sum_{i \in I} \varphi_{i}(\xi)=1$ for all $\xi \in \mathcal{O}$;

(iii) $\varphi_{i}(\xi)=0$ for all $\xi \in \mathcal{O} \backslash Q_{i}$ and all $i \in I$;

(iv) $C_{\Phi}:=\sup _{i \in I}\left\|\mathcal{F}^{-1} \varphi_{i}\right\|_{L^{1}}<\infty$.

The cover $\mathcal{Q}$ is called a decomposition cover if there exists a BAPU subordinate to $\mathcal{Q}$.

Given a decomposition cover $\mathcal{Q}=\left(Q_{i}\right)_{i \in I}$ of an open set $\emptyset \neq \mathcal{O} \subset \widehat{\mathbb{R}}^{d}$, it will be assumed throughout this article that a BAPU $\Phi=\left(\varphi_{i}\right)_{i \in I}$ for $\mathcal{Q}=\left(Q_{i}\right)_{i \in I}$ is fixed.

Definition 3.4 Let $\mathcal{O} \neq \emptyset$ be an open subset of $\widehat{\mathbb{R}}^{d}$. A family $\mathcal{Q}=\left(Q_{i}\right)_{i \in I}$ of subsets $Q_{i} \subset \mathcal{O}$ is called an affinely generated cover of $\mathcal{O}$ if, for each $i \in I$, there are $A_{i} \in \mathrm{GL}(d, \mathbb{R})$ and $b_{i} \in \widehat{\mathbb{R}}^{d}$ and an open subset $Q_{i}^{\prime} \subset \widehat{\mathbb{R}}^{d}$ with $Q_{i}=A_{i}\left(Q_{i}^{\prime}\right)+b_{i}$ satisfying the following:

(i) $\mathcal{Q}$ is an admissible cover of $\mathcal{O}$;

(ii) the sets $\left(Q_{i}^{\prime}\right)_{i \in I}$ are uniformly bounded, that is,

$$
R_{\mathcal{Q}}:=\sup _{i \in I} \sup _{\xi \in Q_{i}^{\prime}}|\xi|<\infty
$$

(iii) for indices $i, \ell \in I$ with $Q_{i} \cap Q_{\ell} \neq \emptyset$, the transformations $A_{i}(\cdot)+b_{i}$ and $A_{\ell}(\cdot)+b_{\ell}$ are uniformly compatible, that is,

$$
C_{\mathcal{Q}}:=\sup _{i \in I} \sup _{\ell \in i^{*}}\left\|A_{i}^{-1} A_{\ell}\right\|<\infty
$$

and moreover, for each $i \in I$, there is an open set $Q_{i}^{\prime \prime} \subset \widehat{\mathbb{R}}^{d}$ such that

(iv) the closure $\overline{Q_{i}^{\prime \prime}} \subset Q_{i}^{\prime}$ for all $i \in I$;

(v) the family $\left(A_{i}\left(Q_{i}^{\prime \prime}\right)+b_{i}\right)_{i \in I}$ covers $\mathcal{O}$; and

(vi) the sets $\left\{Q_{i}^{\prime}: i \in I\right\}$ and $\left\{Q_{i}^{\prime \prime}: i \in I\right\}$ are finite.

Remark 3.5 An affinely generated cover is also called an (almost) structured cover in the literature, see for instance [61] and [11] for similar notions.

In the sequel, the map $S_{i}: \widehat{\mathbb{R}}^{d} \rightarrow \widehat{\mathbb{R}}^{d}$ will always denote an affine linear mapping $\xi \mapsto A_{i} \xi+b_{i}$ for some $A_{i} \in \mathrm{GL}(d, \mathbb{R})$ and $b_{i} \in \widehat{\mathbb{R}}^{d}$.

Definition 3.6 Let $\mathcal{Q}=\left(S_{i}\left(Q_{i}^{\prime}\right)\right)_{i \in I}$ be an affinely generated cover of $\mathcal{O}$, and let $\Phi=\left(\varphi_{i}\right)_{i \in I}$ be a smooth partition of unity subordinate to $\mathcal{Q}$. For $i \in I$, define the normalization of $\varphi_{i}$ by $\varphi_{i}^{b}:=\varphi_{i} \circ S_{i}$. The family $\Phi=\left(\varphi_{i}\right)_{i \in I}$ is called a regular partition of unity, subordinate to $\mathcal{Q}$, if

$$
C_{\mathcal{Q}, \Phi, \alpha}:=\sup _{i \in I}\left\|\partial^{\alpha} \varphi_{i}^{b}\right\|_{L^{\infty}}<\infty
$$

for all multi-indices $\alpha \in \mathbb{N}_{0}^{d}$. 
The following result shows that every affinely generated cover is a decomposition cover.

Proposition 3.7 ([60, Corollary 2.7 and Theorem 2.8]) Let $\mathcal{Q}=\left(S_{i}\left(Q_{i}^{\prime}\right)\right)_{i \in I}$ be an affinely generated cover of $\mathcal{O}$. Then, the following hold:

(1) Every regular partition of unity $\Phi$ subordinate to $\mathcal{Q}$ is also a BAPU subordinate to $\mathcal{Q}$.

(2) There exists a regular partition of unity $\Phi=\left(\varphi_{i}\right)_{i \in I}$ subordinate to $\mathcal{Q}$.

\subsection{Besov-Type Spaces}

We introduce Besov-type spaces following the approach in [56], which relies on the space of Fourier distributions. Since we only treat the Besov-type scale of spaces, we allow for rather general covers. More restrictions would be necessary to include the Triebel-Lizorkin scale, because the corresponding theory relies on inequalities for maximal functions; see [55,Sect. 3.6], [56,Sect. 2.4.3], and also [47].

Definition 3.8 Let $\mathcal{O} \neq \varnothing$ be open in $\widehat{\mathbb{R}}^{d}$. The space $Z(\mathcal{O}):=\mathcal{F}\left(C_{c}^{\infty}(\mathcal{O})\right)$ is called the Fourier test function space on $\mathcal{O}$. The space $Z(\mathcal{O})$ is endowed with the unique topology making the Fourier transform $\mathcal{F}: C_{c}^{\infty}(\mathcal{O}) \rightarrow Z(\mathcal{O})$ into a homeomorphism.

The topological dual space $(Z(\mathcal{O}))^{\prime}$ of $Z(\mathcal{O})$ is denoted by $Z^{\prime}(\mathcal{O})$ and is called the space of Fourier distributions. The (bilinear) dual pairing between $Z^{\prime}(\mathcal{O})$ and $Z(\mathcal{O})$ will be denoted by $\langle\phi, f\rangle_{Z^{\prime}, Z}:=\langle\phi, f\rangle_{Z^{\prime}}:=\langle\phi, f\rangle:=\phi(f)$ for $\phi \in Z^{\prime}(\mathcal{O})$ and $f \in Z(\mathcal{O})$.

The Fourier transform $\phi \in \mathcal{D}^{\prime}(\mathcal{O})$ of a Fourier distribution $\phi \in Z^{\prime}(\mathcal{O})$ is defined by duality; i.e.,

$$
\mathcal{F}: Z^{\prime}(\mathcal{O}) \rightarrow \mathcal{D}^{\prime}(\mathcal{O}), \quad \phi \mapsto \mathcal{F} \phi:=\widehat{\phi}:=\phi \circ \mathcal{F},
$$

which entails $\langle\mathcal{F} \phi, f\rangle_{\mathcal{D}^{\prime}, \mathcal{D}}=\langle\phi, \mathcal{F} f\rangle_{Z^{\prime}, Z}$ for $\phi \in Z^{\prime}(\mathcal{O})$ and $f \in C_{c}^{\infty}(\mathcal{O})$.

Using the Fourier distributions as a reservoir, a decomposition space is defined as follows:

Definition 3.9 Let $p, q \in[1, \infty]$. Let $\mathcal{Q}=\left(Q_{i}\right)_{i \in I}$ be a decomposition cover of an open set $\emptyset \neq \mathcal{O} \subset \widehat{\mathbb{R}}^{d}$ with associated BAPU $\left(\varphi_{i}\right)_{i \in I}$. Let $w=\left(w_{i}\right)_{i \in I}$ be $\mathcal{Q}$ moderate. For $f \in Z^{\prime}(\mathcal{O})$, set

$$
\|f\|_{\mathcal{D}\left(\mathcal{Q}, L^{p}, \ell_{w}^{q}\right)}:=\left\|\left(\left\|\mathcal{F}^{-1}\left(\varphi_{i} \cdot \widehat{f}\right)\right\|_{L^{p}}\right)_{i \in I}\right\|_{\ell_{w}^{q}} \in[0, \infty],
$$

and define the associated decomposition space $\mathcal{D}\left(\mathcal{Q}, L^{p}, \ell_{w}^{q}\right)$ as

$$
\mathcal{D}\left(\mathcal{Q}, L^{p}, \ell_{w}^{q}\right):=\left\{f \in Z^{\prime}(\mathcal{O}):\|f\|_{\mathcal{D}\left(\mathcal{Q}, L^{p}, \ell_{w}^{q}\right)}<\infty\right\}
$$


Remark 3.10 The norm (3.2) is well-defined: If $f \in Z^{\prime}(\mathcal{O})$, then $\widehat{f} \in \mathcal{D}^{\prime}(\mathcal{O})$, whence $\varphi_{i} \cdot \widehat{f}$ is a (tempered) distribution with compact support. By the PaleyWiener theorem [54,Theorem 7.23], it follows therefore that $\mathcal{F}^{-1}\left(\varphi_{i} \cdot \widehat{f}\right)$ is given by a smooth function. In addition, $\mathcal{D}\left(\mathcal{Q}, L^{p}, \ell_{w}^{q}\right)$ is a Banach space and independent of the choice of the BAPU $\left(\varphi_{i}\right)_{i \in I}$, with equivalent norms for different choices; see [61,Corollary 3.18 and Theorem 3.21].

Remark 3.11 Our presentation follows [61, 62] and relies on the original approach of $[56,58]$, specially in the use of Fourier distributions, which is essential for the more technical aspects of our results. More abstract versions of Besov-type spaces replace the Fourier transform by an adequate symmetric operator [57] or use a more general Banach space of functions on a locally compact space in lieu of the Fourier image of $L^{p}$ [23]. This latter (far reaching) generalization is particularly useful to model signal processing applications, such as sampling.

In the sequel, we will often prove our results on the subspace $\mathcal{S}_{\mathcal{O}}\left(\mathbb{R}^{d}\right):=$ $\mathcal{F}^{-1}\left(C_{c}^{\infty}(\mathcal{O})\right) \subset \mathcal{S}\left(\mathbb{R}^{d}\right)$ of the space $\mathcal{D}\left(\mathcal{Q}, L^{p}, \ell_{w}^{q}\right)$, and then extend to all of $\mathcal{D}\left(\mathcal{Q}, L^{p}, \ell_{w}^{q}\right)$ by a suitable density argument. These density arguments rely on the following concept.

Definition 3.12 Let $I$ be an index set, and let $w=\left(w_{i}\right)_{i \in I}$ be a weight. For a sequence $F=\left(F_{i}\right)_{i \in I}$ of functions $F_{i} \in L^{p}\left(\mathbb{R}^{d}\right)$, we write $\|F\|_{\ell_{w}^{q}\left(I ; L^{p}\right)}:=\left\|\left(\left\|F_{i}\right\|_{L^{p}}\right)_{i \in I}\right\|_{\ell_{w}^{q}} \in$ $[0, \infty]$, and set

$$
\ell_{w}^{q}\left(I ; L^{p}\right):=\left\{F \in\left[L^{p}\left(\mathbb{R}^{d}\right)\right]^{I}:\|F\|_{\ell_{w}^{q}\left(I ; L^{p}\right)}<\infty\right\}
$$

Let $\mathcal{Q}=\left(Q_{i}\right)_{i \in I}$ be a decomposition cover of an open set $\mathcal{O} \subset \widehat{\mathbb{R}}^{d}$ with BAPU $\Phi=\left(\varphi_{i}\right)_{i \in I}$, and let $F=\left(F_{i}\right)_{i \in I}$ be a family of functions $F_{i}: \mathbb{R}^{d} \rightarrow[0, \infty)$. A Fourier distribution $f \in Z^{\prime}(\mathcal{O})$ is said to be $(F, \Phi)$-dominated if, for all $i \in I$,

$$
\left|\mathcal{F}^{-1}\left(\varphi_{i} \cdot \widehat{f}\right)\right| \leq F_{i}
$$

We next state our density result; its proof is postponed to Appendix B.

Proposition 3.13 Let $\mathcal{Q}=\left(Q_{i}\right)_{i \in I}$ be a decomposition cover of an open set $\emptyset \neq \mathcal{O} \subset$ $\widehat{\mathbb{R}}^{d}$ with BAPU $\Phi=\left(\varphi_{i}\right)_{i \in I}$ and let $w=\left(w_{i}\right)_{i \in I}$ be a $\mathcal{Q}$-moderate weight. Then

(i) The inclusion $\mathcal{S}_{\mathcal{O}}\left(\mathbb{R}^{d}\right) \subset \mathcal{D}\left(\mathcal{Q}, L^{p}, \ell_{w}^{q}\right)$ holds for all $p, q \in[1, \infty]$.

(ii) If $p, q \in[1, \infty)$, then $\mathcal{S}_{\mathcal{O}}\left(\mathbb{R}^{d}\right)$ is norm dense in $\mathcal{D}\left(\mathcal{Q}, L^{p}, \ell_{w}^{q}\right)$.

(iii) If $p, q \in[1, \infty]$ and $f \in \mathcal{D}\left(\mathcal{Q}, L^{p}, \ell_{w}^{q}\right)$, then there exist $F \in \ell_{w}^{q}\left(I ; L^{p}\right)$ satisfying

$$
\|F\|_{\ell_{w}^{q}\left(I ; L^{p}\right)} \leq C_{\Phi}\left\|\Gamma_{\mathcal{Q}}\right\|_{\ell_{w}^{q} \rightarrow \ell_{w}^{q}}^{2} \cdot\|f\|_{\mathcal{D}\left(\mathcal{Q}, L^{p}, \ell_{w}^{q}\right)},
$$

and a sequence $\left(g_{n}\right)_{n \in \mathbb{N}}$ of $(F, \Phi)$-dominated functions $g_{n} \in \mathcal{S}_{\mathcal{O}}\left(\mathbb{R}^{d}\right)$ such that $g_{n} \rightarrow f$, with convergence in $Z^{\prime}(\mathcal{O})$. 
Remark 3.14 The inclusion $\mathcal{S}_{\mathcal{O}}\left(\mathbb{R}^{d}\right) \subset \mathcal{D}\left(\mathcal{Q}, L^{p}, \ell_{w}^{q}\right) \subset Z^{\prime}(\mathcal{O})$ in Proposition 3.13(i) should be understood in the following sense: Clearly $\mathcal{S}_{\mathcal{O}}\left(\mathbb{R}^{d}\right) \subset \mathcal{S}\left(\mathbb{R}^{d}\right) \hookrightarrow \mathcal{S}^{\prime}\left(\mathbb{R}^{d}\right)$, where as usual a function $f \in \mathcal{S}\left(\mathbb{R}^{d}\right)$ is identified with the distribution $\phi \mapsto \int f \cdot \phi d x$. But since $Z(\mathcal{O}) \hookrightarrow \mathcal{S}\left(\mathbb{R}^{d}\right)$, each $f \in \mathcal{S}^{\prime}\left(\mathbb{R}^{d}\right)$ restricts to an element of $Z^{\prime}(\mathcal{O})$; in particular, each $f \in \mathcal{S}_{\mathcal{O}}$ can be seen as an element of $Z^{\prime}(\mathcal{O})$ by virtue of $\langle f, \phi\rangle_{Z^{\prime}, Z}=$ $\int f \cdot \phi d x$. Under this identification, the Fourier transform $\mathcal{F} f \in \mathcal{D}^{\prime}(\mathcal{O})$ is just the usual $\widehat{f} \in \mathcal{S}\left(\widehat{\mathbb{R}}^{d}\right)$, interpreted as a distribution on $\mathcal{O}$.

As a companion to the above density result, the following Fatou property of the decomposition spaces $\mathcal{D}\left(\mathcal{Q}, L^{p}, \ell_{w}^{q}\right)$ will be used. For the proof, see [31,Lemma 36].

Lemma 3.15 Let $\mathcal{Q}=\left(Q_{i}\right)_{i \in I}$ be a decomposition cover of an open set $\emptyset \neq \mathcal{O} \subset$ $\widehat{\mathbb{R}}^{d}$. Let $w=\left(w_{i}\right)_{i \in I}$ be a $\mathcal{Q}$-moderate weight, and let $p, q \in[1, \infty]$. Suppose that $\left(f_{n}\right)_{n \in \mathbb{N}}$ is a sequence in $\mathcal{D}\left(\mathcal{Q}, L^{p}, \ell_{w}^{q}\right)$ such that $\lim _{\inf } \rightarrow \infty\left\|f_{n}\right\|_{\mathcal{D}\left(\mathcal{Q}, L^{p}, \ell_{w}^{q}\right)}<\infty$ and $f_{n} \rightarrow f \in Z^{\prime}(\mathcal{O})$, with convergence in $Z^{\prime}(\mathcal{O})$. Then $f \in \mathcal{D}\left(\mathcal{Q}, L^{p}, \ell_{w}^{q}\right)$, with associated norm estimate $\|f\|_{\mathcal{D}\left(\mathcal{Q}, L^{p}, \ell_{w}^{q}\right)} \leq \liminf _{n \rightarrow \infty}\left\|f_{n}\right\|_{\mathcal{D}\left(\mathcal{Q}, L^{p}, \ell_{w}^{q}\right)}$.

\subsection{The Extended Pairing}

We will use the following extension of the $L^{2}$-inner product.

Definition 3.16 Let $\mathcal{Q}=\left(Q_{i}\right)_{i \in I}$ be a decomposition cover of an open set $\emptyset \neq \mathcal{O} \subset$ $\widehat{\mathbb{R}}^{d}$. Let $\Phi=\left(\varphi_{i}\right)_{i \in I}$ be a BAPU subordinate to $\mathcal{Q}$. For $f \in Z^{\prime}(\mathcal{O})$ and $g \in L^{1}\left(\mathbb{R}^{d}\right)$ with $\widehat{g} \in C^{\infty}\left(\widehat{\mathbb{R}}^{d}\right)$, define the extended inner product between $f$ and $g$ as

$$
\langle f \mid g\rangle_{\Phi}:=\sum_{i \in I}\left\langle\widehat{f} \mid \varphi_{i} \cdot \widehat{g}\right\rangle_{\mathcal{D}^{\prime}, \mathcal{D}}
$$

provided that the series on the right-hand side converges absolutely.

Remark 3.17 (i) For $f \in L^{2}\left(\mathbb{R}^{d}\right)$ satisfying $\widehat{f} \equiv 0$ almost everywhere on $\widehat{\mathbb{R}}^{d} \backslash \mathcal{O}$ and for $g \in L^{1}\left(\mathbb{R}^{d}\right) \cap L^{2}\left(\mathbb{R}^{d}\right)$ with $\widehat{g} \in C^{\infty}\left(\widehat{\mathbb{R}}^{d}\right)$, the extended inner product defined above coincides with the standard inner product on $L^{2}$. Indeed, since $\left|\varphi_{i}(\xi)\right| \leq\left\|\varphi_{i}\right\|_{\mathcal{F} L^{1}} \leq C_{\Phi}$ and thus $\sum_{i \in I}\left|\varphi_{i}(\xi)\right| \leq N_{\mathcal{Q}} C_{\Phi}$, we can apply the dominated convergence theorem to see that

$$
\begin{aligned}
\langle f \mid g\rangle_{\Phi} & =\sum_{i \in I}\left\langle\widehat{f} \mid \varphi_{i} \cdot \widehat{g}\right\rangle_{\mathcal{D}^{\prime}, \mathcal{D}}=\sum_{i \in I} \int_{\widehat{\mathbb{R}}^{d}} \widehat{f}(\xi) \overline{\varphi_{i}(\xi)} \overline{\widehat{g}(\xi)} d \xi \\
& =\int_{\widehat{\mathbb{R}}^{d}} \widehat{f}(\xi) \overline{\widehat{g}(\xi)} \overline{\sum_{i \in I} \varphi_{i}(\xi)} d \xi=\int_{\mathcal{O}} \widehat{f}(\xi) \overline{\widehat{g}(\xi)} d \xi \\
& =\langle\widehat{f} \mid \widehat{g}\rangle_{L^{2}}=\langle f \mid g\rangle_{L^{2}} .
\end{aligned}
$$

(ii) In general, it is not clear whether the extended inner product defined above is independent of the chosen BAPU. However, as we will show in Lemma 4.4, the extended pairing is independent of this choice under suitable hypotheses. 


\section{Boundedness of the Frame Operator}

In this section, we present conditions under which the frame operator associated with a generalized shift-invariant system is well-defined and bounded on Besov-type decomposition spaces. These conditions involve the interplay between smoothness and decay of the generators and the underlying frequency cover. See also [52,Sect. 2] and [62] for related estimates.

\subsection{Generalized Shift-Invariant Systems}

Definition 4.1 Let $J$ be a countable index set. For $j \in J$, let $C_{j} \in \operatorname{GL}(d, \mathbb{R})$ and $g_{j} \in L^{2}\left(\mathbb{R}^{d}\right)$. A generalized shift-invariant (GSI) system, associated with $\left(g_{j}\right)_{j \in J}$ and $\left(C_{j}\right)_{j \in J}$, is defined as

$$
\left(T_{\gamma} g_{j}\right)_{j \in J, \gamma \in C_{j} \mathbb{Z}^{d}}=\left(g_{j}(\cdot-\gamma)\right)_{j \in J, \gamma \in C_{j} \mathbb{Z}^{d}}
$$

Throughout the paper, we assume the following standing hypotheses on the system.

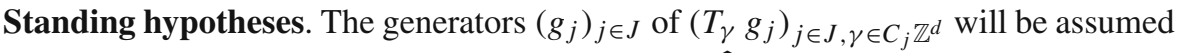
to satisfy $g_{j} \in L^{1}\left(\mathbb{R}^{d}\right) \cap L^{2}\left(\mathbb{R}^{d}\right)$ and $\widehat{g_{j}} \in C^{\infty}\left(\widehat{\mathbb{R}}^{d}\right)$. Moreover, we will use the function $t_{0}:=\sum_{j \in J}\left|\operatorname{det} C_{j}\right|^{-1}\left|\widehat{g}_{j}\right|^{2}$ for which we assume that there exist constants $A, B>0$ such that

$$
A \leq \sum_{j \in J} \frac{1}{\left|\operatorname{det} C_{j}\right|}\left|\widehat{g_{j}}(\xi)\right|^{2} \leq B \quad \text { for a.e. } \xi \in \widehat{\mathbb{R}}^{d} .
$$

Remark 4.2 The assumption (4.1) is automatically satisfied for any generalized shiftinvariant frame $\left(T_{\gamma} g_{j}\right)_{j \in J, \gamma \in C_{j} \mathbb{Z}^{d}}$ for $L^{2}\left(\mathbb{R}^{d}\right)$, with frame bounds $A, B>0$, if it satisfies the so-called $\alpha$-local integrability condition (5.1) introduced below. For a proof, see [30,Theorem 3.13 and Remark 5] and [39,Proposition 4.1].

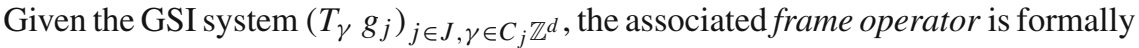
defined as

$$
S: \mathcal{D}\left(\mathcal{Q}, L^{p}, \ell_{w}^{q}\right) \rightarrow \mathcal{D}\left(\mathcal{Q}, L^{p}, \ell_{w}^{q}\right), \quad f \mapsto \sum_{j \in J} \sum_{k \in \mathbb{Z}^{d}}\left\langle f \mid T_{C_{j} k} g_{j}\right\rangle_{\Phi} T_{C_{j} k} g_{j}
$$

For analyzing the boundedness and well-definedness of the frame operator, the following terminology will be convenient.

Definition 4.3 Let $\mathcal{Q}=\left(Q_{i}\right)_{i \in I}$ be a decomposition cover of an open set $\mathcal{O} \subset \widehat{\mathbb{R}}^{d}$ with BAPU $\left(\varphi_{i}\right)_{i \in I}$. Let $w=\left(w_{i}\right)_{i \in I}$ and $v=\left(v_{j}\right)_{j \in J}$ be weights. The system $\left(T_{\gamma} g_{j}\right)_{j \in J, \gamma \in C_{j} \mathbb{Z}^{d}}$ is said to be $(w, v, \Phi)$-adapted if the matrix $M \in \mathbb{C}^{I \times J}$ defined by

$$
M_{i, j}:=\max \left\{\frac{w_{i}}{v_{j}}, \frac{v_{j}}{w_{i}}\right\} \cdot\left|\operatorname{det} C_{j}\right|^{\frac{1}{2}} \cdot\left\|\left(\check{\varphi}_{i} * g_{j}\right) \circ C_{j}\right\|_{W\left(L^{\infty}, \ell^{1}\right)}
$$


is of Schur-type.

Lemma 4.4 Let $\mathcal{Q}=\left(Q_{i}\right)_{i \in I}$ be a decomposition cover with BAPU $\Phi$. Let $w=$ $\left(w_{i}\right)_{i \in I}$ be a $\mathcal{Q}$-moderate weight and let the weight $v=\left(v_{j}\right)_{j \in J}$ be arbitrary.

(i) If $\left(T_{\gamma} g_{j}\right)_{j \in J, \gamma \in C_{j} \mathbb{Z}^{d}}$ is $(w, v, \Phi)$-adapted, then $\left(T_{\gamma} g_{j}\right)_{j \in J, \gamma \in C_{j} \mathbb{Z}^{d}}$ is $(w, v, \Psi)$ adapted for any BAPU $\Psi$ subordinate to $\mathcal{Q}$.

(ii) If $\left(T_{\gamma} g_{j}\right)_{j \in J, \gamma \in C_{j} \mathbb{Z}^{d}}$ is $(w, v, \Phi)$-adapted, then the extended inner product $\langle f|$ $\left.T_{C_{j} k} g_{j}\right\rangle_{\Phi}$ is well-defined and independent of the choice of the BAPU $\Phi$, for any $p, q \in[1, \infty]$, any $f \in \mathcal{D}\left(\mathcal{Q}, L^{p}, \ell_{w}^{q}\right)$, and all $j \in J$ and $k \in \mathbb{Z}^{d}$.

Proof We assume throughout that $\Phi=\left(\varphi_{i}\right)_{i \in I}$ and $\Psi=\left(\psi_{i}\right)_{i \in I}$ are two BAPUs subordinate to $\mathcal{Q}$.

We first show that if $\left(T_{\gamma} g_{j}\right)_{j \in J, \gamma \in C_{j} \mathbb{Z}^{d}}$ is $(w, v, \Phi)$-adapted, then $\left(T_{\gamma} g_{j}\right)_{j \in J, \gamma \in C_{j} \mathbb{Z}^{d}}$ is also $(w, v, \Psi)$-adapted. For this, note that $(f * g)(C x)=|\operatorname{det} C| \cdot((f \circ C) *(g \circ$ $C))(x)$ for any $f \in L^{1}\left(\mathbb{R}^{d}\right), g \in L^{1}\left(\mathbb{R}^{d}\right) \cap L^{\infty}\left(\mathbb{R}^{d}\right)$, and $C \in \operatorname{GL}(d, \mathbb{R})$. Using this, together with $\psi_{i}=\varphi_{i}^{*} \psi_{i}$, yields

$$
\begin{aligned}
\left\|\left(\widetilde{\psi_{i}} * g_{j}\right) \circ C_{j}\right\|_{W\left(L^{\infty}, L^{1}\right)} & \leq \sum_{\ell \in i^{*}}\left\|\left[\left(\mathcal{F}^{-1} \psi_{i}\right) *\left(\widetilde{\varphi_{\ell}} * g_{j}\right)\right] \circ C_{j}\right\|_{W\left(L^{\infty}, L^{1}\right)} \\
& =\sum_{\ell \in i^{*}}\left|\operatorname{det} C_{j}\right| \cdot\left\|\left[\widetilde{\psi_{i}} \circ C_{j}\right] *\left[\left(\widetilde{\varphi_{\ell}} * g_{j}\right) \circ C_{j}\right]\right\|_{W\left(L^{\infty}, L^{1}\right)} \\
& \leq \sum_{\ell \in i^{*}}\left|\operatorname{det} C_{j}\right| \cdot\left\|\widetilde{\psi_{i}} \circ C_{j}\right\|_{L^{1}} \cdot\left\|\left(\check{\varphi}_{\ell} * g_{j}\right) \circ C_{j}\right\|_{W\left(L^{\infty}, L^{1}\right)} \\
& \leq C \cdot C_{\Psi} \cdot \sum_{\ell \in i^{*}}\left\|\left(\check{\varphi}_{\ell} * g_{j}\right) \circ C_{j}\right\|_{W\left(L^{\infty}, \ell^{1}\right)},
\end{aligned}
$$

where $C \geq 1$ is given by the norm equivalence $\|\cdot\|_{W\left(L^{\infty}, \ell^{1}\right)} \asymp\|\cdot\|_{W\left(L^{\infty}, L^{1}\right)}$.

The matrix entries $M_{i, j}$ in (4.2) satisfy

$$
M_{i, j}=\max \left\{\frac{w_{i}}{v_{j}}, \frac{v_{j}}{w_{i}}\right\} \cdot\left|\operatorname{det} C_{j}\right|^{1 / 2} \cdot\left\|\left(\check{\varphi}_{i} * g_{j}\right) \circ C_{j}\right\|_{W\left(L^{\infty}, \ell^{1}\right)} .
$$

Likewise, let us define

$$
N_{i, j}:=\max \left\{\frac{w_{i}}{v_{j}}, \frac{v_{j}}{w_{i}}\right\} \cdot\left|\operatorname{det} C_{j}\right|^{1 / 2} \cdot\left\|\left(\widetilde{\psi_{i}} * g_{j}\right) \circ C_{j}\right\|_{W\left(L^{\infty}, \ell^{1}\right)} .
$$

Using the moderateness of the weight $w$ and the equivalence $\ell \in i^{*} \Longleftrightarrow i \in \ell^{*}$, we obtain that

$$
\begin{aligned}
\sum_{i \in I} N_{i, j} & \leq C^{2} C_{\Psi} \sum_{i \in I} \sum_{\ell \in i^{*}} \max \left\{\frac{w_{i}}{v_{j}}, \frac{v_{j}}{w_{i}}\right\}\left|\operatorname{det} C_{j}\right|^{1 / 2}\left\|\left(\widetilde{\varphi_{\ell}} * g_{j}\right) \circ C_{j}\right\|_{W\left(L^{\infty}, \ell^{1}\right)} \\
& \leq C^{2} C_{\Psi} C_{w, \mathcal{Q}} \cdot \sum_{\ell \in I} \sum_{i \in \ell^{*}} \max \left\{\frac{w_{\ell}}{v_{j}}, \frac{v_{j}}{w_{\ell}}\right\}\left|\operatorname{det} C_{j}\right|^{1 / 2}\left\|\left(\widetilde{\varphi_{\ell}} * g_{j}\right) \circ C_{j}\right\|_{W\left(L^{\infty}, \ell^{1}\right)} \\
& \leq C^{2} C_{\Psi} C_{w, \mathcal{Q}} N_{\mathcal{Q}} \sum_{\ell \in I} M_{\ell, j} \leq C^{2} C_{\Psi} C_{w, \mathcal{Q}} N_{\mathcal{Q}}\|M\|_{\text {Schur }}<\infty
\end{aligned}
$$


for all $j \in J$. Similarly,

$$
\begin{aligned}
\sum_{j \in J} N_{i, j} & \leq C^{2} C_{\Psi} \cdot \sum_{j \in J} \sum_{\ell \in i^{*}} \max \left\{\frac{w_{i}}{v_{j}}, \frac{v_{j}}{w_{i}}\right\}\left|\operatorname{det} C_{j}\right|^{1 / 2}\left\|\left(\check{\varphi}_{\ell} * g_{j}\right) \circ C_{j}\right\|_{W\left(L^{\infty}, \ell^{1}\right)} \\
& \leq C^{2} C_{\Psi} C_{w, \mathcal{Q}} \cdot \sum_{\ell \in i^{*}} \sum_{j \in J} M_{\ell, j} \leq C^{2} C_{\Psi} C_{w, \mathcal{Q}} N_{\mathcal{Q}}\|M\|_{\text {Schur }}<\infty
\end{aligned}
$$

for all $i \in I$. In combination, these two estimates show that $N=\left(N_{i, j}\right)_{i \in I, j \in J}$ is of Schur-type.

Finally, let $p, q \in[1, \infty]$ and $f \in \mathcal{D}\left(\mathcal{Q}, L^{p}, \ell_{w}^{q}\right)$, as well as $j \in J$ and $k \in \mathbb{Z}^{d}$ be arbitrary; we show that the extended product $\left\langle f \mid T_{C_{j} k} g_{j}\right\rangle_{\Phi}$ is well-defined and that $\left\langle f \mid T_{C_{j} k} g_{j}\right\rangle_{\Phi}=\left\langle f \mid T_{C_{j} k} g_{j}\right\rangle_{\Psi}$. To show this, set $B_{j, i}:=\left|\operatorname{det} C_{j}\right|^{1 / 2} \cdot \|\left(\check{\varphi}_{i} *\right.$ $\left.g_{j}\right) \circ C_{j} \|_{W\left(C_{0}, \ell^{1}\right)}$. Since $\left(T_{\gamma} g_{j}\right)_{j \in J, \gamma \in C_{j} \mathbb{Z}^{d}}$ is $(w, v, \Phi)$-adapted, Schur's test shows that $\mathbf{B}: \ell_{w}^{q}(I) \rightarrow \ell_{v}^{q}(J),\left(c_{i}\right)_{i \in I} \mapsto\left(\sum_{i \in I} B_{j, i} c_{i}\right)_{j \in J}$ is well-defined and bounded. Define $d_{i}:=\left\|\mathcal{F}^{-1}\left(\varphi_{i} \cdot \widehat{f}\right)\right\|_{L^{p}}$ and $c_{i}:=\left\|\mathcal{F}^{-1}\left(\varphi_{i}^{*} \cdot \widehat{f}\right)\right\|_{L^{p}}$, and note that $0 \leq c_{i} \leq$ $\sum_{\ell \in i^{*}} d_{\ell}=\left(\Gamma_{\mathcal{Q}} d\right)_{i}$, whence $c=\left(c_{i}\right)_{i \in I} \in \ell_{w}^{q}(I)$, since $d=\left(d_{i}\right)_{i \in I} \in \ell_{w}^{q}(I)$ as $f \in \mathcal{D}\left(\mathcal{Q}, L^{p}, \ell_{w}^{q}\right)$.

As the final setup, let $p^{\prime} \in[1, \infty]$ denote the conjugate exponent to $p$, and set $g:=T_{C_{j} k} g_{j}$. Since $\|f\|_{L^{p^{\prime}}} \leq\|f\|_{W\left(C_{0}, \ell^{1}\right)}$ for all $f \in W\left(C_{0}, \ell^{1}\right)$ and since $\check{\varphi}_{i} * g=T_{C_{j} k}\left(\check{\varphi}_{i} * g_{j}\right)$, it follows that

$$
\begin{aligned}
\left\|\mathcal{F}^{-1}\left(\varphi_{i} \psi_{\ell} \widehat{g}\right)\right\|_{L^{p^{\prime}}} & \leq C_{\Psi} \cdot\left\|\check{\varphi}_{i} * g\right\|_{L^{p^{\prime}}}=C_{\Psi} \cdot\left|\operatorname{det} C_{j}\right|^{1 / p^{\prime}} \cdot\left\|\left(\check{\varphi}_{i} * g_{j}\right) \circ C_{j}\right\|_{L^{p^{\prime}}} \\
& \leq C_{\Psi} \cdot\left|\operatorname{det} C_{j}\right|^{1 / p^{\prime}} \cdot\left\|\left(\check{\varphi}_{i} * g_{j}\right) \circ C_{j}\right\|_{W\left(C_{0}, \ell^{1}\right)} \\
& =C_{\Psi} \cdot\left|\operatorname{det} C_{j}\right|^{\frac{1}{2}-\frac{1}{p}} \cdot B_{j, i}
\end{aligned}
$$

Using that $\varphi_{i}=\varphi_{i}^{*} \varphi_{i}$, and $\widehat{g} \in C^{\infty}\left(\widehat{\mathbb{R}}^{d}\right)$, we next see

$$
\begin{aligned}
\left|\left\langle\widehat{f} \mid \varphi_{i} \psi_{\ell} \widehat{g}\right\rangle_{\mathcal{D}^{\prime}, \mathcal{D}}\right| & =\left|\left\langle\varphi_{i}^{*} \widehat{f} \mid \varphi_{i} \psi_{\ell} \widehat{g}\right\rangle_{\mathcal{S}^{\prime}, \mathcal{S}}\right|=\left|\left\langle\mathcal{F}^{-1}\left(\varphi_{i}^{*} \widehat{f}\right) \mid \mathcal{F}^{-1}\left(\varphi_{i} \psi_{\ell} \widehat{g}\right)\right\rangle_{L^{p}, L^{p^{\prime}}}\right| \\
& \leq\left\|\mathcal{F}^{-1}\left(\varphi_{i}^{*} \widehat{f}\right)\right\|_{L^{p}} \cdot\left\|\mathcal{F}^{-1}\left(\varphi_{i} \psi_{\ell} \widehat{g}\right)\right\|_{L^{p^{\prime}}} \\
& \leq C_{\Psi} \cdot c_{i} \cdot\left|\operatorname{det} C_{j}\right|^{\frac{1}{2}-\frac{1}{p}} \cdot B_{j, i},
\end{aligned}
$$

where the right-hand side is independent of $\ell$. Given this estimate, it follows immediately that

$$
\sum_{i \in I} \sum_{\ell \in i^{*}}\left|\left\langle\widehat{f} \mid \varphi_{i} \psi_{\ell} \widehat{g}\right\rangle_{\mathcal{D}^{\prime}, \mathcal{D}}\right| \leq C_{\Psi} N_{\mathcal{Q}} \cdot\left|\operatorname{det} C_{j}\right|^{\frac{1}{2}-\frac{1}{p}} \cdot(\mathbf{B} c)_{j}<\infty
$$


Therefore, we can interchange the sums in the following calculation:

$$
\begin{aligned}
\langle f \mid g\rangle_{\Phi} & =\sum_{i \in I}\left\langle\widehat{f} \mid \varphi_{i} \widehat{g}\right\rangle_{\mathcal{D}^{\prime}, \mathcal{D}}=\sum_{i \in I} \sum_{\ell \in i^{*}}\left\langle\widehat{f} \mid \varphi_{i} \psi_{\ell} \widehat{g}\right\rangle_{\mathcal{D}^{\prime}, \mathcal{D}} \\
& =\sum_{\ell \in I} \sum_{i \in \ell^{*}}\left\langle\widehat{f} \mid \varphi_{i} \psi_{\ell} \widehat{g}\right\rangle_{\mathcal{D}^{\prime}, \mathcal{D}}=\sum_{\ell \in I}\left\langle\widehat{f} \mid \psi_{\ell} \widehat{g}\right\rangle_{\mathcal{D}^{\prime}, \mathcal{D}}=\langle f \mid g\rangle_{\Psi} .
\end{aligned}
$$

This calculation implies in particular that both $\langle f \mid g\rangle_{\Phi}$ and $\langle f \mid g\rangle_{\Psi}$ are welldefined.

\subsection{Sequence Spaces and Operators}

The frame operator can be factored into the coefficient and the reconstruction operator. In this subsection, we investigate the boundedness of these operators on suitable sequence spaces.

Definition 4.5 Let $\left(T_{\gamma} g_{j}\right)_{j \in J, \gamma \in C_{j} \mathbb{Z}^{d}}$ be a generalized shift-invariant system and let $p, q \in[1, \infty]$. For a weight $v=\left(v_{j}\right)_{j \in J}$ and a sequence $c=\left(c_{k}^{(j)}\right)_{j \in J, k \in \mathbb{Z}^{d}} \in \mathbb{C}^{J \times \mathbb{Z}^{d}}$, define

$$
\|c\|_{Y_{v}^{p, q}}:=\left\|\left(v_{j} \cdot\left|\operatorname{det} C_{j}\right|^{\frac{1}{p}-\frac{1}{2}} \cdot\left\|\left(c_{k}^{(j)}\right)_{k \in \mathbb{Z}^{d}}\right\|_{\ell p}\right)_{j \in J}\right\|_{\ell q} \in[0, \infty] .
$$

Finally, define the associated coefficient space $Y_{v}^{p, q}$ as

$$
Y_{v}^{p, q}:=\left\{c \in \mathbb{C}^{J \times \mathbb{Z}^{d}}:\|c\|_{Y_{v}^{p, q}}<\infty\right\}
$$

Let $\mathcal{D}\left(\mathcal{Q}, L^{p}, \ell_{w}^{q}\right)$ be a decomposition space. Given a GSI system $\left(T_{\gamma} g_{j}\right)_{j \in J, \gamma \in C_{j} \mathbb{Z}^{d}}$ and an associated coefficient space $Y_{v}^{p, q}$, the reconstruction or synthesis operator is formally defined as the mapping

$$
\mathscr{D}: Y_{v}^{p, q} \rightarrow \mathcal{D}\left(\mathcal{Q}, L^{p}, \ell_{w}^{q}\right), \quad\left(c_{k}^{(j)}\right)_{j \in J, k \in \mathbb{Z}^{d}} \mapsto \sum_{j \in J} \sum_{k \in \mathbb{Z}^{d}} c_{k}^{(j)} T_{C_{j} k} g_{j},
$$

while the coefficient or analysis operator is formally defined by

$$
\mathscr{C}: \mathcal{D}\left(\mathcal{Q}, L^{p}, \ell_{w}^{q}\right) \rightarrow Y_{v}^{p, q}, \quad f \mapsto\left(\left\langle f \mid T_{C_{j} k} g_{j}\right\rangle_{\Phi}\right)_{j \in J, k \in \mathbb{Z}^{d}},
$$

where $\langle\cdot, \cdot\rangle_{\Phi}$ denotes the extended pairing defined in Sect. 3.3.

\subsection{Boundedness of Analysis and Synthesis Operators}

For proving the boundedness of the operators $\mathscr{D}$ and $\mathscr{C}$, we will invoke the following lemma. 
Lemma 4.6 Let $g \in W\left(C_{0}, \ell^{1}\right)\left(\mathbb{R}^{d}\right)$ and $M \in \mathrm{GL}\left(\mathbb{R}^{d}\right)$. Then the map

$$
D_{M, g}: c=\left(c_{k}\right)_{k \in \mathbb{Z}^{d}} \mapsto \sum_{k \in \mathbb{Z}^{d}} c_{k} T_{M k} g
$$

is bounded from $\ell^{\infty}\left(\mathbb{Z}^{d}\right)$ into $L^{\infty}\left(\mathbb{R}^{d}\right)$, with the series converging pointwise absolutely. Furthermore, for any $p \in[1, \infty]$, the mapping $D_{M, g}: \ell^{p}\left(\mathbb{Z}^{d}\right) \rightarrow L^{p}\left(\mathbb{R}^{d}\right)$ is welldefined and bounded, with $\left\|D_{M, g}\right\|_{\ell^{p} \rightarrow L^{p}} \leq|\operatorname{det} M|^{1 / p} \cdot\|g \circ M\|_{W\left(L^{\infty}, \ell^{1}\right)}$.

Proof For the case $M=\mathrm{id}_{\mathbb{R}^{d}}$, this follows from [1,Lemma 2.9] —see also [14]. For the general case, simply note that $D_{M, g} c(x)=\left(D_{\mathrm{id}_{\mathbb{R}^{d}}, g \circ M}(c)\right)\left(M^{-1} x\right)$.

The following technical lemma allows us to use density arguments for the full range $p, q \in[1, \infty]$.

Lemma 4.7 Let $p, q \in[1, \infty]$. Suppose the system $\left(T_{\gamma} g_{j}\right)_{j \in J, \gamma \in C_{j} \mathbb{Z}^{d}}$ is $(w, v, \Phi)$ adapted with matrix $M$ as in (4.2). Then, for any $F \in \ell_{w}^{q}\left(I ; L^{p}\right)$, there is a sequence $\theta=\left(\theta_{j, k}\right)_{j \in J, k \in \mathbb{Z}^{d}} \in Y_{v}^{p, q}$ such that

$$
\|\theta\|_{Y_{v}^{p, q}} \leq\|M\|_{\mathrm{Schur}} \cdot\left\|\Gamma_{\mathcal{Q}}\right\|_{\ell_{w}^{q} \rightarrow \ell_{w}^{q}} \cdot\|F\|_{\ell_{w}^{q}\left(I ; L^{p}\right)}
$$

and $\left|\left\langle f \mid T_{C_{j} k} g_{j}\right\rangle_{\Phi}\right| \leq \theta_{j, k}$ for all $j \in J, k \in \mathbb{Z}^{d}$ and every $(F, \Phi)$-dominated $f \in$ $Z^{\prime}(\mathcal{O})$.

Moreover, if $\left(f_{n}\right)_{n \in \mathbb{N}}$ is a sequence of $(F, \Phi)$-dominated Fourier distributions $f_{n} \in Z^{\prime}(\mathcal{O})$ satisfying $f_{n} \rightarrow f_{0} \in Z^{\prime}(\mathcal{O})$ with convergence in $Z^{\prime}(\mathcal{O})$, then $\left\langle f_{n} \mid T_{C_{j} k} g_{j}\right\rangle_{\Phi} \rightarrow\left\langle f_{0} \mid T_{C_{j} k} g_{j}\right\rangle_{\Phi}$ for all $j \in J, k \in \mathbb{Z}^{d}$.

Proof Let $f \in Z^{\prime}(\mathcal{O})$ be $(F, \Phi)$-dominated. Using $\overline{\varphi_{i}^{*}} \varphi_{i}=\varphi_{i}$ and the estimate (3.3), we see that

$$
\begin{aligned}
\left|\left\langle\widehat{f} \mid \varphi_{i} \cdot \mathcal{F}\left[T_{C_{j} k} g_{j}\right]\right\rangle_{\mathcal{D}^{\prime}, \mathcal{D}}\right| & =\left|\left\langle\varphi_{i}^{*} \widehat{f} \mid \mathcal{F}\left[T_{C_{j} k}\left(\breve{\varphi}_{i} * g_{j}\right)\right]\right\rangle_{\mathcal{S}^{\prime}, \mathcal{S}}\right| \\
& \leq \sum_{\ell \in i^{*}}\left|\left\langle\mathcal{F}^{-1}\left(\varphi_{\ell} \widehat{f}\right) \mid T_{C_{j} k}\left(\check{\varphi}_{i} * g_{j}\right)\right\rangle_{\mathcal{S}^{\prime}, \mathcal{S}}\right| \\
& \leq \sum_{\ell \in i^{*}} \int_{\mathbb{R}^{d}} F_{\ell}(x) \cdot\left(T_{C_{j} k}\left|\check{\varphi}_{i} * g_{j}\right|\right)(x) d x=: \sum_{\ell \in i^{*}} \zeta_{i, j, k, \ell},
\end{aligned}
$$

and thus

$$
\left|\left\langle f \mid T_{C_{j} k} g_{j}\right\rangle_{\Phi}\right|=\left|\sum_{i \in I}\left\langle\widehat{f} \mid \varphi_{i} \cdot \mathcal{F}\left[T_{C_{j} k} g_{j}\right]\right\rangle_{\mathcal{D}^{\prime}, \mathcal{D}}\right| \leq \sum_{i \in I} \sum_{\ell \in i^{*}} \zeta_{i, j, k, \ell}=: \theta_{j, k}
$$

with $\zeta_{i, j, k, \ell}$ and $\theta_{j, k}$ being independent of $f$. 
Next, define a measure $\mu_{i, j, k}$ on $\mathbb{R}^{d}$ by $d \mu_{i, j, k}(x):=\left(T_{C_{j} k}\left|\check{\varphi}_{i} * g_{j}\right|\right)(x) d x$. Then

$$
\begin{aligned}
\zeta_{i, j, k, \ell}=\int_{\mathbb{R}^{d}} F_{\ell}(x) \cdot 1 d \mu_{i, j, k}(x) & \leq\left\|F_{\ell}\right\|_{L^{p}\left(\mu_{i, j, k}\right)} \cdot\|1\|_{L^{p^{\prime}}\left(\mu_{i, j, k}\right)} \\
& =\left\|F_{\ell}\right\|_{L^{p}\left(\mu_{i, j, k}\right)} \cdot\left\|T_{C_{j} k}\left(\check{\varphi}_{i} * g_{j}\right)\right\|_{L^{1}}^{1 / p^{\prime}} \\
& \leq\left|\operatorname{det} C_{j}\right|^{1 / p^{\prime}} \cdot\left\|F_{\ell}\right\|_{L^{p}\left(\mu_{i, j, k}\right)} \cdot\left\|\left(\check{\varphi}_{i} * g_{j}\right) \circ C_{j}\right\|_{W\left(L^{\infty}, \ell^{1}\right)}^{1 / p^{\prime}} .
\end{aligned}
$$

There are now two cases. If $p=\infty$, then the estimate (4.7) and $\|\cdot\|_{L^{\infty}\left(\mu_{i, j, k}\right)} \leq$ $\|\cdot\|_{L^{\infty}}$ yield that

$$
\left\|\left(\zeta_{i, j, k, \ell}\right)_{k \in \mathbb{Z}^{d}}\right\|_{\ell^{\infty}} \leq\left|\operatorname{det} C_{j}\right|^{1 / p^{\prime}} \cdot\left\|F_{\ell}\right\|_{L^{p}\left(\mathbb{R}^{d}\right)} \cdot\left\|\left(\check{\varphi}_{i} * g_{j}\right) \circ C_{j}\right\|_{W\left(L^{\infty}, \ell^{1}\right)} \cdot
$$

If $p<\infty$, then (4.7) and Lemma 4.6 together show that

$$
\begin{aligned}
& \sum_{k \in \mathbb{Z}^{d}} \zeta_{i, j, k, \ell}^{p} \leq\left|\operatorname{det} C_{j}\right|^{p / p^{\prime}} \cdot\left\|\left(\check{\varphi}_{i} * g_{j}\right) \circ C_{j}\right\|_{W\left(L^{\infty}, \ell^{1}\right)}^{p / p^{\prime}} \cdot \sum_{k \in \mathbb{Z}^{d}} \int_{\mathbb{R}^{d}}\left(F_{\ell}(x)\right)^{p} \\
& \cdot\left(T_{C_{j} k}\left|\check{\varphi}_{i} * g_{j}\right|\right)(x) d x=\left|\operatorname{det} C_{j}\right|^{p / p^{\prime}} \cdot\left\|\left(\check{\varphi}_{i} * g_{j}\right) \circ C_{j}\right\|_{W\left(L^{\infty}, \ell^{1}\right)}^{p / p^{\prime}} \\
& \cdot \int_{\mathbb{R}^{d}}\left(F_{\ell}(x)\right)^{p} \cdot\left[D_{C_{j},\left|\check{\varphi}_{i} * g_{j}\right|}(1)_{k \in \mathbb{Z}^{d}}\right](x) d x \\
& \leq\left|\operatorname{det} C_{j}\right|^{p / p^{\prime}} \cdot\left\|\left(\check{\varphi}_{i} * g_{j}\right) \circ C_{j}\right\|_{W\left(L^{\infty}, \ell^{1}\right)}^{1+\left(p / p^{\prime}\right)} \cdot\left\|F_{\ell}\right\|_{L^{p}\left(\mathbb{R}^{d}\right)}^{p} \cdot
\end{aligned}
$$

Hence, $\left\|\left(\zeta_{i, j, k, \ell}\right)_{k \in \mathbb{Z}^{d}}\right\|_{\ell^{p}} \leq\left|\operatorname{det} C_{j}\right|^{1 / p^{\prime}} \cdot\left\|\left(\check{\varphi}_{i} * g_{j}\right) \circ C_{j}\right\|_{W\left(L^{\infty}, \ell^{1}\right)} \cdot\left\|F_{\ell}\right\|_{L^{p}}$ for any $p \in[1, \infty]$.

Define $c \in \ell_{w}^{q}(I)$ by $c_{\ell}:=\left\|F_{\ell}\right\|_{L^{p}}$. Then, for all $j \in J$,

$$
\begin{aligned}
v_{j} \mid \operatorname{det} & \left.C_{j}\right|^{\frac{1}{p}-\frac{1}{2}}\left\|\left(\theta_{j, k}\right)_{k \in \mathbb{Z}^{d}}\right\|_{\ell p} \\
\leq & \sum_{i \in I} \sum_{\ell \in i^{*}} v_{j}\left|\operatorname{det} C_{j}\right|^{\frac{1}{p}-\frac{1}{2}}\left\|\left(\zeta_{i, j, k, \ell}\right)_{k \in \mathbb{Z}^{d}}\right\|_{\ell p} \\
\leq & \sum_{i \in I}\left[v_{j}\left|\operatorname{det} C_{j}\right|^{\frac{1}{p}-\frac{1}{2}}\left|\operatorname{det} C_{j}\right|^{1-\frac{1}{p}}\left\|\left(\check{\varphi}_{i} * g_{j}\right) \circ C_{j}\right\|_{W\left(L^{\infty}, \ell^{1}\right)} \sum_{\ell \in i^{*}} c_{\ell}\right] \\
\leq & \sum_{i \in I} M_{i, j} \cdot w_{i} \cdot\left(\Gamma_{\mathcal{Q}} c\right)_{i}
\end{aligned}
$$

where $M_{i, j}$ is defined as in Eq. (4.2). Next, since $\left(T_{\gamma} g_{j}\right)_{j \in J, \gamma \in C_{j} \mathbb{Z}^{d}}$ is $(w, v, \Phi)$ adapted, Schur's test shows that $\mathbf{M}: \ell^{q}(I) \rightarrow \ell^{q}(J),\left(d_{i}\right)_{i \in I} \mapsto\left(\sum_{i \in I} M_{i, j} d_{i}\right)_{j \in J}$ is well-defined and bounded, with norm $\|\mathbf{M}\|_{\ell q \rightarrow \ell q} \leq\|M\|_{\text {Schur }}$. Consequently, we obtain

$$
\left\|\left(\theta_{j, k}\right)_{j \in J, k \in \mathbb{Z}^{d}}\right\|_{Y_{v}^{p, q}} \leq\left\|\mathbf{M}\left(w \cdot \Gamma_{\mathcal{Q}}(c)\right)\right\|_{\ell^{q}(J)} \leq\|M\|_{\text {Schur }} \cdot\left\|\Gamma_{\mathcal{Q}}\right\|_{\ell_{w}^{q} \rightarrow \ell_{w}^{q}} \cdot\|c\|_{\ell_{w}^{q}} \cdot
$$


But $\|c\|_{\ell_{w}^{q}}=\|F\|_{\ell_{w}^{q}\left(I ; L^{p}\right)}$, and thus the first part of the proof is complete.

For the proof of the second part, first note

$$
\left\langle\widehat{f}_{n} \mid \varphi_{i} \cdot \mathcal{F}\left[T_{C_{j} k} g_{j}\right]\right\rangle_{\mathcal{D}^{\prime}, \mathcal{D}} \underset{n \rightarrow \infty}{\longrightarrow}\left\langle\widehat{f}_{0} \mid \varphi_{i} \cdot \mathcal{F}\left[T_{C_{j} k} g_{j}\right]\right\rangle_{\mathcal{D}^{\prime}, \mathcal{D}}
$$

since $\varphi_{i} \cdot \mathcal{F}\left[T_{C_{j} k} g_{j}\right] \in C_{c}^{\infty}(\mathcal{O})$ and since $f_{n} \rightarrow f_{0}$ in $Z^{\prime}(\mathcal{O})$ which implies $\widehat{f}_{n} \rightarrow \widehat{f}_{0}$ in $\mathcal{D}^{\prime}(\mathcal{O})$. Next, since the $f_{n}$ are $(F, \Phi)$-dominated, Eq. (4.5) shows that

$\left|\left\langle\widehat{f}_{n} \mid \varphi_{i} \cdot \mathcal{F}\left[T_{C_{j} k} g_{j}\right]\right\rangle_{\mathcal{D}^{\prime}, \mathcal{D}}\right| \leq \sum_{\ell \in i^{*}} \zeta_{i, j, k, \ell} \leq u_{j}^{-1} \sum_{\ell \in i^{*}} u_{j}\left\|\left(\zeta_{i, j, k, \ell}\right)_{k \in \mathbb{Z}^{d}}\right\|_{\ell^{p}}=: \gamma_{i, j}$

while Eq. (4.8) shows that $\sum_{i \in I} \gamma_{i, j}<\infty$. Thus,

$$
\left\langle f_{n} \mid T_{C_{j} k} g_{j}\right\rangle_{\Phi} \underset{n \rightarrow \infty}{\longrightarrow}\left\langle f_{0} \mid T_{C_{j} k} g_{j}\right\rangle_{\Phi}
$$

by definition of $\langle\cdot \mid \cdot\rangle_{\Phi}$ and by the dominated convergence theorem.

We now prove the boundedness of the coefficient and reconstruction operators.

Proposition 4.8 Let $\mathcal{D}\left(\mathcal{Q}, L^{p}, \ell_{w}^{q}\right)$ be a decomposition space and let $Y_{v}^{p, q}$ be the

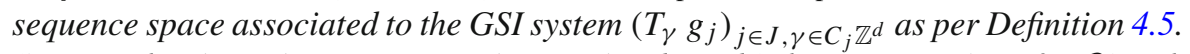
Suppose that $\left(T_{\gamma} g_{j}\right)_{j \in J, \gamma \in C_{j} \mathbb{Z}^{d}}$ is $(w, v, \Phi)$-adapted (where $\Phi$ is a BAPU for $\mathcal{Q}$ ) with matrix $M$ as in (4.2). Then

(i) For all $p, q \in[1, \infty]$, the reconstruction map

$$
\mathscr{D}: Y_{v}^{p, q} \rightarrow \mathcal{D}\left(\mathcal{Q}, L^{p}, \ell_{w}^{q}\right), \quad\left(c_{k}^{(j)}\right)_{j \in J, k \in \mathbb{Z}^{d}} \mapsto \sum_{j \in J} \sum_{k \in \mathbb{Z}^{d}} c_{k}^{(j)} \cdot T_{C_{j} k} g_{j}
$$

is well-defined and bounded with $\|\mathscr{D}\|_{Y_{v}^{p, q} \rightarrow \mathcal{D}\left(\mathcal{Q}, L^{p}, \ell_{w}^{q}\right)} \leq\|M\|_{\text {Schur. Furthermore, }}$ the defining double series converges unconditionally in $Z^{\prime}(\mathcal{O})$.

(ii) For all $p, q \in[1, \infty]$, the coefficient operator

$$
\mathscr{C}: \mathcal{D}\left(\mathcal{Q}, L^{p}, \ell_{w}^{q}\right) \rightarrow Y_{v}^{p, q}, \quad f \mapsto\left(\left\langle f \mid T_{C_{j} k} g_{j}\right\rangle_{\Phi}\right)_{j \in J, k \in \mathbb{Z}^{d}}
$$

is well-defined and bounded with $\|\mathscr{C}\|_{\mathcal{D}\left(\mathcal{Q}, L^{p}, \ell_{w}^{q}\right) \rightarrow Y_{v}^{p, q}} \leq\|M\|_{\operatorname{Schur}} \cdot\left\|\Gamma_{\mathcal{Q}}\right\|_{\ell_{w}^{q} \rightarrow \ell_{w}^{q}}$. (iii) If $\Psi$ is another BAPU for $\mathcal{Q}$, and if $f \in \mathcal{D}\left(\mathcal{Q}, L^{p}, \ell_{w}^{q}\right)$, then $\left\langle f \mid T_{C_{j} k} g_{j}\right\rangle_{\Psi}$ is well-defined and satisfies $\left\langle f \mid T_{C_{j} k} g_{j}\right\rangle_{\Psi}=\left\langle f \mid T_{C_{j} k} g_{j}\right\rangle_{\Phi}$ for all $j \in J$ and $k \in \mathbb{Z}^{d}$.

Proof To prove (i), let $c=\left(c_{k}^{(j)}\right)_{j \in J, k \in \mathbb{Z}^{d}} \in Y_{v}^{p, q}$ be arbitrary, and set $c^{(j)}:=$ $\left(c_{k}^{(j)}\right)_{k \in \mathbb{Z}^{d}}$ for $j \in J$. Then $c^{(j)} \in \ell^{p}\left(\mathbb{Z}^{d}\right)$. Moreover, if $d=\left(d_{j}\right)_{j \in J}$ is defined as $d_{j}:=\left|\operatorname{det} C_{j}\right|^{\frac{1}{p}-\frac{1}{2}} \cdot\left\|c^{(j)}\right\|_{\ell^{p}}$, then $d \in \ell_{v}^{q}(J)$ and $\|d\|_{\ell_{v}^{q}}=\|c\|_{Y_{v}^{p}, q}$. Finally, let $\left|c^{(j)}\right|=\left(\left|c_{k}^{(j)}\right|\right)_{k \in \mathbb{Z}^{d}}$ for $j \in J$. 
We first prove the unconditional convergence of the double series defining $\mathscr{D} c$. Since the Fourier transform $\mathcal{F}: Z^{\prime}(\mathcal{O}) \rightarrow \mathcal{D}^{\prime}(\mathcal{O})$ is a linear homeomorphism, it suffices to show that the double series $\sum_{j \in J} \sum_{k \in \mathbb{Z}^{d}} c_{k}^{(j)} \mathcal{F}\left[T_{C_{j} k} g_{j}\right]$ converges unconditionally in $\mathcal{D}^{\prime}(\mathcal{O})$. To prove this, let $K \subset \mathcal{O}$ be compact. Since $\sum_{i \in I} \varphi_{i} \equiv 1$ on $\mathcal{O}$, the family $\left(\varphi_{i}^{-1}(\mathbb{C} \backslash\{0\})\right)_{i \in I}$ forms an open cover of $\mathcal{O} \supset K$. By compactness of $K$, there is a finite set $I_{K} \subset I$ for which $K \subset \bigcup_{i \in I_{K}} \varphi_{i}^{-1}(\mathbb{C} \backslash\{0\}) \subset \bigcup_{i \in I_{K}} Q_{i}$. Note that $I_{K}^{*}:=\bigcup_{\ell \in I_{K}} \ell^{*} \subset I$ is finite. Furthermore, for $j \in I \backslash I_{K}^{*}$, note that $Q_{j} \cap K \subset \bigcup_{i \in I_{K}} Q_{j} \cap Q_{i}=\emptyset$, whence $\varphi_{j} \equiv 0$ on $K$. Thus, any $g \in C_{c}^{\infty}(\mathcal{O}) \subset \mathcal{S}\left(\widehat{\mathbb{R}}^{d}\right)$ with supp $g \subset K$ can be written as $g=\sum_{i \in I} \varphi_{i} g=\sum_{i \in I_{K}^{*}} \varphi_{i} g$. A direct calculation using Lemma 4.6 therefore shows

$$
\begin{aligned}
& \sum_{j \in J} \sum_{k \in \mathbb{Z}^{d}}\left|c_{k}^{(j)}\right| \cdot\left|\left\langle\mathcal{F}\left[T_{C_{j} k} g_{j}\right], g\right\rangle_{\mathcal{D}^{\prime}, \mathcal{D}}\right| \\
& \quad \leq \sum_{i \in I_{K}^{*}} \sum_{j \in J} \sum_{k \in \mathbb{Z}^{d}}\left|c_{k}^{(j)}\right| \cdot\left|\left\langle\varphi_{i} \mathcal{F}\left[T_{C_{j} k} g_{j}\right], g\right\rangle_{\mathcal{S}^{\prime}, \mathcal{S}}\right| \\
& \quad \leq \sum_{i \in I_{K}^{*}} \sum_{j \in J} \int_{\mathbb{R}^{d}}|\widehat{g}(x)| \sum_{k \in \mathbb{Z}^{d}}\left|c_{k}^{(j)}\right|\left(T_{C_{j} k}\left|\check{\varphi}_{i} * g_{j}\right|\right)(x) d x \\
& \quad \leq \sum_{i \in I_{K}^{*}} \sum_{j \in J}\|\widehat{g}\|_{L^{p^{\prime}}} \cdot\left\|D_{C_{j},\left|\breve{\varphi}_{i} * g_{j}\right|}\left|c^{(j)}\right|\right\|_{L^{p}} \\
& \quad \leq\|\widehat{g}\|_{L^{p^{\prime}}} \sum_{i \in I_{K}^{*}} \sum_{j \in J}\left|\operatorname{det} C_{j}\right|^{\frac{1}{p}}\left\|\left(\check{\varphi}_{i} * g_{j}\right) \circ C_{j}\right\|_{W\left(L^{\infty}, \ell^{1}\right)}\left\|\left|c^{(j)}\right|\right\|_{\ell p} \\
& \leq\|\widehat{g}\|_{L^{p^{\prime}}} \cdot \sum_{i \in I_{K}^{*}}\left[w_{i}^{-1} \sum_{j \in J} v_{j} d_{j} M_{i, j}\right] \\
& \leq\|\widehat{g}\|_{L^{p^{\prime}}} \cdot\|d\|_{\ell_{v}^{q}} \cdot\|M\|_{S c h u r} \cdot \sum_{i \in I_{K}^{*}} w_{i}^{-1}<\infty
\end{aligned}
$$

Since $g \mapsto\|\widehat{g}\|_{L^{p^{\prime}}}$ is a continuous norm on $C_{c}^{\infty}(\mathcal{O})$ and since $g \in C_{c}^{\infty}(\mathcal{O})$ with supp $g \subset K$ was arbitrary, the desired unconditional convergence follows.

Next, we show that $\mathscr{D}: Y_{v}^{p, q} \rightarrow \mathcal{D}\left(\mathcal{Q}, L^{p}, \ell_{w}^{q}\right)$ is well-defined and bounded. For $i \in I$ and $j \in J$, define $B_{i, j}:=\left|\operatorname{det} C_{j}\right|^{\frac{1}{2}} \cdot\left\|\left(\check{\varphi}_{i} * g_{j}\right) \circ C_{j}\right\|_{W\left(L^{\infty}, \ell^{1}\right)}$. The assumption that $\left(T_{\gamma} g_{j}\right)_{j \in J, \gamma \in C_{j} \mathbb{Z}^{d}}$ is $(w, v, \Phi)$-adapted yields by Schur's test that the map $\mathbf{B}: \ell_{v}^{q}(J) \rightarrow \ell_{w}^{q}(I), \quad\left(d_{j}\right)_{j \in J} \mapsto\left(\sum_{j \in J} B_{i, j} \cdot d_{j}\right)_{i \in I}$ is bounded with $\|\mathbf{B}\|_{\text {op }} \leq\|M\|_{\text {Schur. }}$. The series defining $\mathscr{D} c$ being unconditionally convergent yields

$$
\mathcal{F}^{-1}\left(\varphi_{i} \cdot \widehat{\mathscr{D} c}\right)=\sum_{j \in J} \sum_{k \in \mathbb{Z}^{d}} c_{k}^{(j)} \mathcal{F}^{-1}\left(\varphi_{i} \mathcal{F}\left[T_{C_{j} k} g_{j}\right]\right)=\sum_{j \in J} D_{C_{j}, \breve{\varphi}_{i} * g_{j}} c^{(j)}
$$


Therefore, an application of Lemma 4.6 shows

$$
\begin{aligned}
\left\|\mathcal{F}^{-1}\left(\varphi_{i} \cdot \widehat{\mathscr{D} c}\right)\right\|_{L^{p}} & \leq \sum_{j \in J}\left|\operatorname{det} C_{j}\right|^{\frac{1}{p}} \cdot\left\|\left(\check{\varphi}_{i} * g_{j}\right) \circ C_{j}\right\|_{W\left(L^{\infty}, \ell^{1}\right)} \cdot\left\|c^{(j)}\right\|_{\ell^{p}} \\
& =\sum_{j \in J} B_{i, j} d_{j}=(\mathbf{B} d)_{i}<\infty
\end{aligned}
$$

whence $\|\mathscr{D} c\|_{\mathcal{D}\left(\mathcal{Q}, L^{p}, \ell_{w}^{q}\right)} \leq\|\mathbf{B} d\|_{\ell_{w}^{q}} \leq\|M\|_{\text {Schur }} \cdot\|d\|_{\ell_{v}^{q}}=\|M\|_{\text {Schur }} \cdot\|c\|_{Y_{v}^{p, q}}$.

To prove (ii), let $f \in \mathcal{D}\left(\mathcal{Q}, L^{p}, \ell_{w}^{q}\right)$ be arbitrary. Define $F_{i}:=\left|\mathcal{F}^{-1}\left(\varphi_{i} \widehat{f}\right)\right|$ for $i \in I$. Then, $F=\left(F_{i}\right)_{i \in I} \in \ell_{w}^{q}\left(I ; L^{p}\right)$ and $\|F\|_{\ell_{w}^{q}\left(I ; L^{p}\right)}=\|f\|_{\mathcal{D}\left(\mathcal{Q}, L^{p}, \ell_{w}^{q}\right)}$. Clearly, $f$ is $(F, \Phi)$-dominated. Therefore, Lemma 4.7 yields $\theta=\left(\theta_{j, k}\right)_{j \in J, k \in \mathbb{Z}^{d}} \in Y_{v}^{p, q}$ satisfying the estimate $\left|\left\langle f \mid T_{C_{j} k} g_{j}\right\rangle_{\Phi}\right| \leq \theta_{j, k}$ for all $j \in J$ and $k \in \mathbb{Z}^{d}$, and furthermore $\|\theta\|_{Y_{v}^{p, q}} \leq\|M\|_{\text {Schur }} \cdot\left\|\Gamma_{\mathcal{Q}}\right\|_{\ell_{w}^{q} \rightarrow \ell_{w}^{q}} \cdot\|F\|_{\ell_{w}^{q}\left(I ; L^{p}\right)}$. Hence, $\mathscr{C}: \mathcal{D}\left(\mathcal{Q}, L^{p}, \ell_{w}^{q}\right) \rightarrow Y_{v}^{p, q}$ is well-defined and bounded, with the claimed estimate for the operator norm.

Assertion (iii) is a direct consequence of Lemma 4.4.

Proposition 4.8 shows in particular that the reconstruction operator $\mathscr{D}: Y_{v}^{p, q} \rightarrow$ $\mathcal{D}\left(\mathcal{Q}, L^{p}, \ell_{w}^{q}\right)$ is continuous. However, in case $\max \{p, q\}=\infty$, the convergence in $Y_{v}^{p, q}$ is a quite restrictive condition. To accommodate for this, we will often employ the following lemma.

Lemma 4.9 Under the assumptions of Proposition 4.8, the following holds:

For each $n \in \mathbb{N}$, let $c^{(n)}=\left(c_{j, k}^{(n)}\right)_{j \in J, k \in \mathbb{Z}^{d}} \in Y_{v}^{p, q}$ be such that $c_{j, k}^{(n)} \underset{n \rightarrow \infty}{\longrightarrow} c_{j, k} \in \mathbb{C}$ for all $j \in J$ and $k \in \mathbb{Z}^{d}$. Suppose there exists a sequence $\theta=\left(\theta_{j, k}\right)_{j \in J, k \in \mathbb{Z}^{d}} \in Y_{v}^{p, q}$ satisfying $\left|c_{j, k}^{(n)}\right| \leq \theta_{j, k}$ for all $j \in J, k \in \mathbb{Z}^{d}$, and $n \in \mathbb{N}$. Then, the reconstruction operator $\mathscr{D}$ satisfies $\mathscr{D} c^{(n)} \underset{n \rightarrow \infty}{\stackrel{Z^{\prime}(\mathcal{O})}{\longrightarrow}} \mathscr{D} c$.

Proof Let $f \in Z(\mathcal{O})$. Then $K:=\operatorname{supp} \mathcal{F}^{-1} f \subset \mathcal{O}$ is compact. Since $\left(\varphi_{i}^{-1}(\mathbb{C} \backslash\right.$ $\{0\}))_{i \in I}$ is an open cover of $K$, there is a finite set $I_{0} \subset I$ satisfying $K \subset \bigcup_{i \in I_{0}} \varphi_{i}^{-1}(\mathbb{C} \backslash$ \{0\}) $\subset \bigcup_{i \in I_{0}} Q_{i}$. This easily implies $Q_{i} \cap K=\emptyset$ for $i \in I \backslash I_{f}$, where $I_{f}:=$ $I_{0}^{*}:=\bigcup_{\ell \in I_{0}} \ell^{*} \subset I$ is finite. Thus, $\varphi_{i} \cdot \mathcal{F}^{-1} f \equiv 0$ for $i \in I \backslash I_{f}$, and hence $\mathcal{F}^{-1} f=\sum_{i \in I_{f}} \varphi_{i} \mathcal{F}^{-1} f$. Therefore,

$$
\begin{aligned}
\left\langle T_{C_{j} k} g_{j}, f\right\rangle_{\mathcal{S}^{\prime}, \mathcal{S}} & =\left\langle\mathcal{F}\left[T_{C_{j} k} g_{j}\right], \mathcal{F}^{-1} f\right\rangle_{\mathcal{S}^{\prime}, \mathcal{S}}=\sum_{i \in I_{f}}\left\langle\varphi_{i} \mathcal{F}\left[T_{C_{j} k} g_{j}\right], \mathcal{F}^{-1} f\right\rangle_{\mathcal{S}^{\prime}, \mathcal{S}} \\
& =\sum_{i \in I_{f}}\left\langle T_{C_{j} k}\left(\check{\varphi}_{i} * g_{j}\right), f\right\rangle_{\mathcal{S}^{\prime}, \mathcal{S}}
\end{aligned}
$$


For $v=\left(v_{j, k}\right)_{j \in J, k \in \mathbb{Z}^{d}} \in Y_{v}^{p, q}$, it follows, therefore, by the convergence in $Z^{\prime}(\mathcal{O})$ of the series defining $\mathscr{D} v$ that

$$
\begin{aligned}
& \langle\mathscr{D} v, f\rangle_{Z^{\prime}, Z}=\sum_{j \in J} \sum_{k \in \mathbb{Z}^{d}} v_{j, k}\left\langle T_{C_{j} k} g_{j}, f\right\rangle_{\mathcal{S}^{\prime}, \mathcal{S}} \\
& =\sum_{i \in I_{f}} \sum_{j \in J} \sum_{k \in \mathbb{Z}^{d}} v_{j, k}\left\langle T_{C_{j} k}\left(\check{\varphi}_{i} * g_{j}\right), f\right\rangle_{\mathcal{S}^{\prime}, \mathcal{S}} .
\end{aligned}
$$

Next, Lemma 4.6 shows that

$$
\begin{aligned}
\sum_{k \in \mathbb{Z}^{d}} \theta_{j, k}\left|\left\langle T_{C_{j} k}\left(\check{\varphi_{i}} * g_{j}\right), f\right\rangle_{\mathcal{S}^{\prime}, \mathcal{S}}\right| & \leq \int_{\mathbb{R}^{d}}|f(x)| \cdot \sum_{k \in \mathbb{Z}^{d}}\left[\theta_{j, k} \cdot\left(T_{C_{j} k}\left|\check{\varphi_{i}} * g_{j}\right|\right)(x)\right] d x \\
& \leq\|f\|_{L^{p^{\prime}}} \cdot\left\|D_{C_{j},\left|\check{\varphi_{i}} * g_{j}\right|}\left(\left(\theta_{j, k}\right)_{k \in \mathbb{Z}^{d}}\right)\right\|_{L^{p}} \\
& \leq\|f\|_{L^{p^{\prime}}} \cdot\left|\operatorname{det} C_{j}\right|^{1 / p} \cdot\left\|\left(\check{\varphi}_{i} * g_{j}\right) \circ C_{j}\right\|_{W\left(L^{\infty}, \ell^{1}\right)} \cdot \gamma_{j},
\end{aligned}
$$

where we defined $\gamma_{j}:=\left\|\left(\theta_{j, k}\right)_{k \in \mathbb{Z}^{d}}\right\|_{\ell^{p}}$ in the last step.

For brevity, let $u_{j}:=v_{j} \cdot\left|\operatorname{det} C_{j}\right|^{\frac{1}{p}-\frac{1}{2}}$. Note that since $\theta \in Y_{v}^{p, q}$, we have $\gamma=$ $\left(\gamma_{j}\right)_{j \in J} \in \ell_{u}^{q} \hookrightarrow \ell_{u}^{\infty}$, which yields a constant $C_{1}>0$ such that $u_{j} \gamma_{j} \leq C_{1}$ for all $j \in J$. Using this, we see

$$
\begin{aligned}
& \sum_{i \in I_{f}} \sum_{j \in J} \sum_{k \in \mathbb{Z}^{d}} \theta_{j, k}\left|\left\langle T_{C_{j} k}\left(\check{\varphi}_{i} * g_{j}\right), f\right\rangle_{\mathcal{S}^{\prime}, \mathcal{S}}\right| \\
\leq & \|f\|_{L^{p^{\prime}}} \sum_{i \in I_{f}}\left[w_{i}^{-1} \sum_{j \in J} \frac{w_{i}}{v_{j}} \cdot\left|\operatorname{det} C_{j}\right|^{\frac{1}{2}} \cdot\left\|\left(\check{\varphi}_{i} * g_{j}\right) \circ C_{j}\right\|_{W\left(L^{\infty}, \ell^{1}\right)} \cdot u_{j} \gamma_{j}\right] \\
\leq & C_{1} \cdot\|f\|_{L^{p^{\prime}}} \sum_{i \in I_{f}} \sum_{j \in J} \frac{M_{i, j}}{w_{i}} \leq C_{1} \cdot\|f\|_{L^{p^{\prime}}} \cdot\left(\sum_{i \in I_{f}} w_{i}^{-1}\right) \cdot\|M\|_{\text {Schur }}<\infty .
\end{aligned}
$$

Finally, since $\left|c_{j, k}^{(n)}\right| \leq \theta_{j, k}$ for all $j \in J, k \in \mathbb{Z}^{d}$, and $n \in \mathbb{N}$, and since $c_{j, k}^{(n)} \underset{n \rightarrow \infty}{\longrightarrow} c_{j, k}$, applying the dominated convergence theorem in Eq. (4.10) shows that

$$
\left\langle\mathscr{D} c^{(n)}, f\right\rangle_{Z^{\prime}, Z} \underset{n \rightarrow \infty}{\longrightarrow}\langle\mathscr{D} c, f\rangle_{Z^{\prime}, Z}
$$

as desired.

Corollary 4.10 Under the assumptions of Proposition 4.8, the following holds: The frame operator $S:=\mathscr{D} \circ \mathscr{C}: \mathcal{D}\left(\mathcal{Q}, L^{p}, \ell_{w}^{q}\right) \rightarrow \mathcal{D}\left(\mathcal{Q}, L^{p}, \ell_{w}^{q}\right)$ is well-defined and bounded.

Furthermore, if $\left(f_{n}\right)_{n \in \mathbb{N}} \subset \mathcal{D}\left(\mathcal{Q}, L^{p}, \ell_{w}^{q}\right)$ is a sequence satisfying $f_{n} \rightarrow f \in$ $Z^{\prime}(\mathcal{O})$, with convergence in $Z^{\prime}(\mathcal{O})$, and for which there exists $F \in \ell_{w}^{q}\left(I ; L^{p}\right)$ such that all $f_{n}$ are $(F, \Phi)$-dominated, then $f \in \mathcal{D}\left(\mathcal{Q}, L^{p}, \ell_{w}^{q}\right)$ and $S f_{n} \rightarrow S f$ with convergence in $Z^{\prime}(\mathcal{O})$. 
Proof $S$ is well-defined, bounded by Proposition 4.8. Since $\left\|f_{n}\right\|_{\mathcal{D}\left(\mathcal{Q}, L^{p}, \ell_{w}^{q}\right)} \leq$ $\|F\|_{\ell_{w}^{q}\left(I ; L^{p}\right)}$ for all $n \in \mathbb{N}$, Lemma 3.15 yields $f \in \mathcal{D}\left(\mathcal{Q}, L^{p}, \ell_{w}^{q}\right)$, where $c:=\mathscr{C} f \in$ $Y_{v}^{p, q}$. Next, Lemma 4.7 shows that there is a sequence $\theta=\left(\theta_{j, k}\right)_{j \in J, k \in \mathbb{Z}^{d}} \in Y_{v}^{p, q}$ such that if we set $c^{(n)}:=\mathscr{C} f_{n}$, then $\left|c_{j, k}^{(n)}\right| \leq \theta_{j, k}$ for all $(n, j, k) \in \mathbb{N} \times J \times \mathbb{Z}^{d}$. The same lemma also shows that $c_{j, k}^{(n)} \rightarrow c_{j, k}$ for all $j \in J$ and $k \in \mathbb{Z}^{d}$. Therefore, Lemma 4.9 shows that $S f_{n}=\mathscr{D} c^{(n)} \rightarrow \mathscr{D} c=S f$ with convergence in $Z^{\prime}(\mathcal{O})$.

\section{Invertibility of the Frame Operator}

\subsection{Representation of the Frame Operator}

The frame properties of generalized shift-invariant systems are usually studied under a compatibility condition that controls the interaction between the generating functions and the translation lattices of the system. Specifically, we will use the so-called local integrability conditions [39, 41, 59].

Definition 5.1 For an open set $\mathcal{O} \subset \widehat{\mathbb{R}}^{d}$ of full measure, let

$$
\mathcal{B}_{\mathcal{O}}\left(\mathbb{R}^{d}\right):=\left\{f \in L^{2}\left(\mathbb{R}^{d}\right): \widehat{f} \in L^{\infty}\left(\widehat{\mathbb{R}}^{d}\right) \text { and supp } \widehat{f} \subset \mathcal{O} \text { compact }\right\}
$$

A generalized shift-invariant system $\left(T_{\gamma} g_{j}\right)_{j \in J, \gamma \in C_{j} \mathbb{Z}^{d}}$ is said to satisfy the $\alpha$-local integrability condition $(\alpha-L I C)$, relative to $\mathcal{O}^{c}$, if, for all $f \in \mathcal{B}_{\mathcal{O}}\left(\mathbb{R}^{d}\right)$,

$$
\sum_{j \in J} \frac{1}{\left|\operatorname{det} C_{j}\right|} \sum_{\alpha \in C_{j}^{-t} \mathbb{Z}^{d}} \int_{\widehat{\mathbb{R}}^{d}}\left|\widehat{f}(\xi) \widehat{f}(\xi+\alpha) \widehat{g}_{j}(\xi) \widehat{g}_{j}(\xi+\alpha)\right| d \xi<\infty
$$

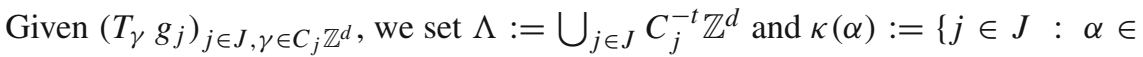
$\left.C_{j}^{-t} \mathbb{Z}^{d}\right\}$ for $\alpha \in \Lambda$. For $\alpha \in \Lambda$, we define the functions

$$
t_{\alpha}: \widehat{\mathbb{R}}^{d} \rightarrow \mathbb{C}, \quad \xi \mapsto \sum_{j \in \kappa(\alpha)} \frac{1}{\left|\operatorname{det} C_{j}\right|} \overline{\widehat{g}_{j}(\xi)} \widehat{g_{j}}(\xi+\alpha)
$$

Note that $t_{\alpha} \in L^{\infty}\left(\widehat{\mathbb{R}}^{d}\right)$ for all $\alpha \in \Lambda$ by (4.1). Furthermore, $t_{\alpha}(\xi-\alpha)=\overline{t_{-\alpha}(\xi)}$.

Under the $\alpha$-local integrability condition, the following (weak-sense) representation of the frame operator can be obtained; this follows by polarization from the proofs of [39,Proposition 2.4] and [41, Theorem 3.4]. 


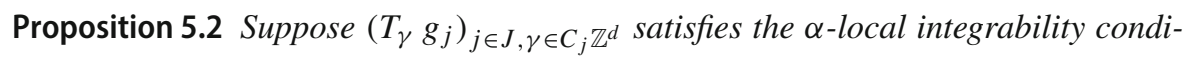
tion (5.1), relative to $\mathcal{O}^{c}$. Then, for all $f_{1}, f_{2} \in \mathcal{B}_{\mathcal{O}}\left(\mathbb{R}^{d}\right)$,

$$
\begin{aligned}
\sum_{(j, k) \in J \times \mathbb{Z}^{d}}\left\langle f_{1} \mid T_{C_{j} k} g_{j}\right\rangle\left\langle T_{C_{j} k} g_{j} \mid f_{2}\right\rangle & =\sum_{\alpha \in \Lambda} \int_{\widehat{\mathbb{R}}^{d}} \widehat{f_{1}}(\xi) \overline{\hat{f}_{2}(\xi+\alpha)} t_{\alpha}(\xi) d \xi \\
& =\sum_{\alpha \in \Lambda}\left\langle\mathcal{F}^{-1}\left[T_{\alpha}\left(t_{\alpha} \widehat{f_{1}}\right)\right] \mid f_{2}\right\rangle_{L^{2}}
\end{aligned}
$$

where the series converges absolutely; in fact,

$$
\sum_{\alpha \in \Lambda} \int_{\widehat{\mathbb{R}}^{d}}\left|\widehat{f_{1}}(\xi) \widehat{f_{2}}(\xi+\alpha)\right| \sum_{j \in \kappa(\alpha)} \frac{1}{\left|\operatorname{det} C_{j}\right|}\left|\widehat{g_{j}}(\xi) \widehat{g_{j}}(\xi+\alpha)\right| d \xi<\infty
$$

Proposition 5.2 yields an analogous representation of the frame operator on $\mathcal{D}\left(\mathcal{Q}, L^{p}, \ell_{w}^{q}\right)$, at least on the subspace $\mathcal{S}_{\mathcal{O}}\left(\mathbb{R}^{d}\right)$.

Corollary 5.3 Under the assumptions of Proposition 5.2, the series $\sum_{\alpha \in \Lambda_{0}} \mathcal{F}^{-1}\left[T_{\alpha}\right.$ $\left(t_{\alpha} \widehat{f}\right)$ ] converges unconditionally in $Z^{\prime}(\mathcal{O})$ for any subset $\Lambda_{0} \subset \Lambda$, and any $f \in$ $\mathcal{S}_{\mathcal{O}}\left(\mathbb{R}^{d}\right)$.

Furthermore, if $\mathcal{Q}$ is a decomposition cover of $\mathcal{O}$, with subordinate $B A P U \Phi$, if $w$ is $\mathcal{Q}$-moderate, and if $v=\left(v_{j}\right)_{j \in J}$ is a weight such that $\left(T_{\gamma} g_{j}\right)_{j \in J, \gamma \in C_{j} \mathbb{Z}^{d}}$ is $(w, v, \Phi)$ adapted, then the frame operator $S: \mathcal{D}\left(\mathcal{Q}, L^{p}, \ell_{w}^{q}\right) \rightarrow \mathcal{D}\left(\mathcal{Q}, L^{p}, \ell_{w}^{q}\right)$ fulfills for each $f \in \mathcal{S}_{\mathcal{O}}\left(\mathbb{R}^{d}\right)$ the identity

$$
\begin{array}{r}
S f=\sum_{j \in J} \sum_{k \in \mathbb{Z}^{d}}\left\langle f \mid T_{C_{j} k} g_{j}\right\rangle_{\Phi} T_{C_{j} k} g_{j}=\sum_{j \in J} \sum_{k \in \mathbb{Z}^{d}}\left\langle f \mid T_{C_{j} k} g_{j}\right\rangle_{L^{2}} T_{C_{j} k} g_{j} \\
=\sum_{\alpha \in \Lambda} \mathcal{F}^{-1}\left[T_{\alpha}\left(t_{\alpha} \widehat{f}\right)\right] .
\end{array}
$$

Proof Since $t_{\alpha} \in L^{\infty}\left(\widehat{\mathbb{R}}^{d}\right)$ and $\widehat{f} \in \mathcal{S}\left(\widehat{\mathbb{R}}^{d}\right)$, we have $T_{\alpha}\left(t_{\alpha} \widehat{f}\right) \in L^{1}\left(\widehat{\mathbb{R}}^{d}\right) \hookrightarrow$ $\mathcal{S}^{\prime}\left(\widehat{\mathbb{R}}^{d}\right) \hookrightarrow \mathcal{D}^{\prime}(\mathcal{O})$, and hence $\mathcal{F}^{-1}\left[T_{\alpha}\left(t_{\alpha} \widehat{f}\right)\right] \in Z^{\prime}(\mathcal{O})$. The Fourier transform $\mathcal{F}: Z^{\prime}(\mathcal{O}) \rightarrow \mathcal{D}^{\prime}(\mathcal{O})$ is a linear homeomorphism; hence, it suffices to prove that the series $\sum_{\alpha \in \Lambda_{0}} T_{\alpha}\left(t_{\alpha} \widehat{f}\right)$ converges unconditionally in $\mathcal{D}^{\prime}(\mathcal{O})$. To see this, let $K \subset \mathcal{O}$ be compact. Define $f_{1}:=f \in \mathcal{S}_{\mathcal{O}}\left(\mathbb{R}^{d}\right) \subset \mathcal{B}_{\mathcal{O}}\left(\mathbb{R}^{d}\right)$, and set $f_{2}:=\mathcal{F}^{-1} \mathbb{1}_{K} \in \mathcal{B}_{\mathcal{O}}\left(\mathbb{R}^{d}\right)$. By Eq. (5.4), the constant $C_{K}:=\sum_{\alpha \in \Lambda} \int_{\widehat{\mathbb{R}}^{d}}|\widehat{f}(\xi)| \mathbb{1}_{K}(\xi+\alpha)\left|t_{\alpha}(\xi)\right| d \xi$ is finite. Now, let $\psi \in C_{c}^{\infty}(\mathcal{O})$ be arbitrary with supp $\psi \subset K$. Then

$$
\begin{aligned}
\sum_{\alpha \in \Lambda_{0}}\left|\left\langle T_{\alpha}\left(t_{\alpha} \widehat{f}\right), \psi\right\rangle_{\mathcal{D}^{\prime}, \mathcal{D}}\right| & \leq \sum_{\alpha \in \Lambda}\|\psi\|_{L^{\infty}} \int_{\widehat{\mathbb{R}}^{d}}\left|t_{\alpha}(\eta-\alpha) \widehat{f}(\eta-\alpha)\right| \cdot \mathbb{1}_{K}(\eta) d \eta \\
& =C_{K}\|\psi\|_{L^{\infty}}<\infty
\end{aligned}
$$

Since $\|\cdot\|_{L^{\infty}}$ is continuous with respect to the topology on $C_{c}^{\infty}(\mathcal{O})$, and since $\psi \in$ $C_{c}^{\infty}(\mathcal{O})$ with supp $\psi \subset K$ was arbitrary, the estimate (5.6) simultaneously yields 
that $\sum_{\alpha \in \Lambda_{0}} T_{\alpha}\left(t_{\alpha} \widehat{f}\right) \in \mathcal{D}^{\prime}(\mathcal{O})$, cf. [54, Theorem 6.6], as well as the unconditional convergence of the series in $\mathcal{D}^{\prime}(\mathcal{O})$.

For the remaining part, note if $f \in \mathcal{S}_{\mathcal{O}}\left(\mathbb{R}^{d}\right)$, then $\left\langle f \mid T_{C_{j} k} g_{j}\right\rangle_{\Phi}=\left\langle f \mid T_{C_{j} k} g_{j}\right\rangle_{L^{2}}$ by Remark 3.17. This proves everything but the last equality in Eq. (5.5). To prove this, let $g \in Z(\mathcal{O})$. Then $\widehat{\bar{g}}=\overline{\mathcal{F}-1} g \in C_{c}^{\infty}(\mathcal{O})$, and hence $\bar{g} \in \mathcal{B}_{\mathcal{O}}\left(\mathbb{R}^{d}\right)$. This, together with Eq. (5.3), shows

$$
\begin{aligned}
\langle S f, g\rangle_{Z^{\prime}, Z}=\langle S f \mid \bar{g}\rangle_{L^{2}} & =\sum_{\alpha \in \Lambda}\left\langle\mathcal{F}^{-1}\left[T_{\alpha}\left(t_{\alpha} \widehat{f}\right)\right] \mid \bar{g}\right\rangle_{L^{2}} \\
= & \left\langle\sum_{\alpha \in \Lambda} \mathcal{F}^{-1}\left[T_{\alpha}\left(t_{\alpha} \widehat{f}\right)\right], g\right\rangle_{Z^{\prime}, Z}
\end{aligned}
$$

and hence (5.5) follows.

\subsection{Towards Invertibility}

According to Corollary 5.3, on the set $\mathcal{S}_{\mathcal{O}}\left(\mathbb{R}^{d}\right)$, the frame operator can be represented as

$$
S f=T_{0} f+R f
$$

with

$$
T_{0} f=\mathcal{F}^{-1}\left(t_{0} \cdot \widehat{f}\right)
$$

and

$$
R f=\mathcal{F}^{-1}\left(\sum_{\alpha \in \Lambda \backslash\{0\}} T_{\alpha}\left(t_{\alpha} \cdot \widehat{f}\right)\right),
$$

for $f \in \mathcal{S}_{\mathcal{O}}\left(\mathbb{R}^{d}\right)$. In the following, we estimate the norms of $T_{0}^{-1}$ and $R$ as operators on the decomposition space $\mathcal{D}\left(\mathcal{Q}, L^{p}, \ell_{w}^{q}\right)$. This will be used, together with the following elementary result, to provide conditions ensuring that the frame operator is invertible.

Lemma 5.4 Let $X$ be a Banach space, and let $S: X \rightarrow X$ be a linear operator that can be written as $S=T_{0}+R$, where $T_{0}, R$ are bounded linear operators on $X$. Finally, assume that $T_{0}$ is boundedly invertible and that

$$
\left\|T_{0}^{-1}\right\|_{X \rightarrow X} \cdot\|R\|_{X \rightarrow X}<1 .
$$

Then, $S: X \rightarrow X$ is also boundedly invertible.

Proof We have $S=T_{0}+R=T_{0}\left(\operatorname{id}_{X}-\left(-T_{0}^{-1} R\right)\right)$. But $\left\|-T_{0}^{-1} R\right\|_{X \rightarrow X} \leq$ $\left\|T_{0}^{-1}\right\|_{X \rightarrow X} \cdot\|R\|_{X \rightarrow X}<1$, so that $\operatorname{id}_{X}-\left(-T_{0}^{-1} R\right)$ is boundedly invertible by a Neumann series argument. This implies that $S$ is boundedly invertible as a composition of boundedly invertible operators. 


\subsection{Estimates for Fourier Multipliers}

The operator $T_{0}$ is a Fourier multiplier, and we aim to estimate its inverse. As a first step, we prove a general result concerning the boundedness of Fourier multipliers on Besovtype spaces; see Proposition 5.7 below. More qualitative versions of that proposition can be found in [56,Sect. 2.4.3], [58,Sect. 2.3] and [23, Theorem 2.11]. Corresponding results for Triebel-Lizorkin spaces hold under more stringent assumptions on the decomposition cover; see [56,Sects. 2.4.2 and 2.5.4] and [55].

In contrast to [56,Sect. 2.4.3], we consider Fourier symbols with limited regularity. This entails certain technical difficulties because of our choice of the reservoir $Z^{\prime}(\mathcal{O})$, where $Z(\mathcal{O})=\mathcal{F}\left(C_{c}^{\infty}(\mathcal{O})\right)$. More precisely, if $f \in \mathcal{D}\left(\mathcal{Q}, L^{p}, \ell_{w}^{q}\right) \subset Z^{\prime}(\mathcal{O})$, then $\widehat{f} \in \mathcal{D}^{\prime}(\mathcal{O})$ is a distribution, and can be multiplied by a function $h \in C^{\infty}(\mathcal{O})$. We need, however, to make sense of the product with more general functions $h$, by fully exploiting the fact that $f \in \mathcal{D}\left(\mathcal{Q}, L^{p}, \ell_{w}^{q}\right)$. To this end, we introduce the following notion:

Definition 5.5 Let $p \in[1, \infty]$. For $f \in \mathcal{F} L^{1}\left(\mathbb{R}^{d}\right)$ and $g \in \mathcal{F} L^{p}\left(\mathbb{R}^{d}\right)$, we define the generalized product of $f$ and $g$ as

$$
f \odot g:=\mathcal{F}\left[\left(\mathcal{F}^{-1} f\right) *\left(\mathcal{F}^{-1} g\right)\right] \in \mathcal{F} L^{p}\left(\mathbb{R}^{d}\right) \subset \mathcal{S}^{\prime}\left(\mathbb{R}^{d}\right) .
$$

Remark 5.6 The definition makes sense because of Young's inequality: $\left(\mathcal{F}^{-1} f\right) *$ $\left(\mathcal{F}^{-1} g\right) \in L^{p}\left(\mathbb{R}^{d}\right)$. Furthermore, our definition indeed generalizes the usual product: if $f \in \mathcal{S}\left(\mathbb{R}^{d}\right)$ and $g \in \mathcal{S}^{\prime}\left(\mathbb{R}^{d}\right)$, then $f \cdot g=\mathcal{F}\left[\left(\mathcal{F}^{-1} f\right) *\left(\mathcal{F}^{-1} g\right)\right]$ - see, for instance [54, Theorem 7.19].

We can now derive an estimate for Fourier multipliers on decomposition spaces. The proof is deferred to Appendix C.

Proposition 5.7 Let $\mathcal{Q}=\left(Q_{i}\right)_{i \in I}$ be a decomposition cover of an open set $\emptyset \neq \mathcal{O} \subset$ $\widehat{\mathbb{R}}^{d}$, and let $\left(\varphi_{i}\right)_{i \in I}$ be a BAPU subordinate to $\mathcal{Q}$. A continuous function $h \in C(\mathcal{O})$ is called tame if

$$
C_{h}:=\sup _{i \in I}\left\|\mathcal{F}^{-1}\left(\varphi_{i} \cdot h\right)\right\|_{L^{1}}<\infty
$$

If $h$ is tame and if $f \in \mathcal{D}\left(\mathcal{Q}, L^{p}, \ell_{w}^{q}\right)$ for certain $p, q \in[1, \infty]$ and a $\mathcal{Q}$-moderate weight $w$, then the series

$$
\Phi_{h} f:=\sum_{i \in I} \mathcal{F}^{-1}\left[\left(\varphi_{i}^{*} h\right) \odot\left(\varphi_{i} \widehat{f}\right)\right]
$$

converges unconditionally in $Z^{\prime}(\mathcal{O})$. Furthermore, the operator $\Phi_{h}$ satisfies the following properties:

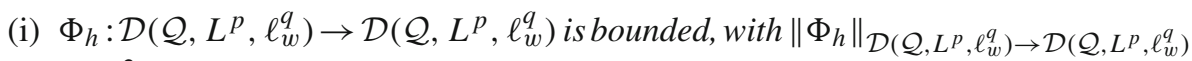
$\leq N_{\mathcal{Q}}^{2} C_{\Phi} C_{h}$ for arbitrary $p, q \in[1, \infty]$ and any $\mathcal{Q}$-moderate weight $w$. 
(ii) If $\left(f_{n}\right)_{n \in \mathbb{N}} \subset Z^{\prime}(\mathcal{O})$ is $(F, \Phi)$-dominated for some $F \in \ell_{w}^{q}\left(I ; L^{p}\right)$ and if $f_{n} \rightarrow f$ with convergence in $Z^{\prime}(\mathcal{O})$, then also $\Phi_{h} f_{n} \rightarrow \Phi_{h} f$ with convergence in $Z^{\prime}(\mathcal{O})$. In addition, there is $G \in \ell_{w}^{q}\left(I ; L^{p}\right)$ such that $\Phi_{h} f_{n}$ is $(G, \Phi)$-dominated for all $n \in \mathbb{N}$ and such that $\|G\|_{\ell_{w}^{q}\left(I ; L^{p}\right)} \leq N_{\mathcal{Q}}^{2} C_{\Phi} C_{h} \cdot\left\|F_{\ell}\right\|_{\ell_{w}^{q}\left(I ; L^{p}\right)}$.

(iii) If $f \in \mathcal{D}\left(\mathcal{Q}, L^{p}, \ell_{w}^{q}\right)$ and $\widehat{f} \in C_{c}(\mathcal{O})$, then $\Phi_{h} f=\mathcal{F}^{-1}(h \cdot \widehat{f})$.

(iv) If $g, h \in C(\mathcal{O})$ are tame, then so is $g \cdot h$, and we have $\Phi_{h} \Phi_{g}=\Phi_{g h}$.

Remark One can show that if $C_{h}$ is finite for one BAPU $\left(\varphi_{i}\right)_{i \in I}$, then the same holds for any other BAPU. Still, the precise value of the constant $C_{h}$ depends on the choice of the BAPU.

\subsection{Estimates for the Remainder Term $R$}

The following proposition provides a general condition under which $R$ defines a bounded operator on $\mathcal{D}\left(\mathcal{Q}, L^{p}, \ell_{w}^{q}\right)$. Simplified versions of these are derived in Sect. 6.

Proposition 5.8 Let $\mathcal{Q}=\left(Q_{i}\right)_{i \in I}$ be a decomposition cover of an open set $\mathcal{O} \subset \widehat{\mathbb{R}}^{d}$ of full measure, with associated BAPU $\Phi=\left(\varphi_{i}\right)_{i \in I}$. Let $w=\left(w_{i}\right)_{i \in I}$ be $\mathcal{Q}$-moderate.

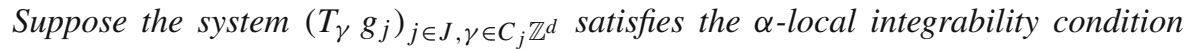
(5.1), with respect to $\mathcal{O}^{c}$. Moreover, suppose that, for all $i, \ell \in I$,

$$
N_{i, \ell}:=\frac{w_{i}}{w_{\ell}} \sum_{\alpha \in \Lambda \backslash\{0\}}\left\|\mathcal{F}^{-1}\left(\varphi_{i}(\cdot+\alpha) \cdot t_{\alpha} \cdot \varphi_{\ell}\right)\right\|_{L^{1}}<\infty
$$

and that the matrix $N=\left(N_{i, \ell}\right)_{i, \ell \in I} \in \mathbb{C}^{I \times I}$ is of Schur-type. Then, for all $p, q \in$ $[1, \infty]$, the "remainder operator $R$ " defined in (5.9) satisfies

$$
\|R f\|_{\mathcal{D}\left(\mathcal{Q}, L^{p}, \ell_{w}^{q}\right)} \leq\|N\|_{\text {Schur }}\left\|\Gamma_{\mathcal{Q}}\right\|_{\ell_{w}^{q}(I) \rightarrow \ell_{w}^{q}(I)}\|f\|_{\mathcal{D}\left(\mathcal{Q}, L^{p}, \ell_{w}^{q}\right)} \quad \forall f \in \mathcal{S}_{\mathcal{O}}\left(\mathbb{R}^{d}\right) .
$$

Proof The assumptions yield, by Schur's test, that the operator

$$
\mathbf{N}: \ell_{w}^{q}(I) \rightarrow \ell_{w}^{q}(I), \quad\left(c_{\ell}\right)_{\ell \in I} \mapsto\left(\sum_{\ell \in I}\left[\sum_{\alpha \in \Lambda \backslash\{0\}}\left\|\varphi_{i}(\cdot+\alpha) \cdot t_{\alpha} \cdot \varphi_{\ell}\right\|_{\mathcal{F} L^{1}}\right] \cdot c_{\ell}\right)_{i \in I},
$$

is bounded, with $\|\mathbf{N}\|_{\ell_{w}^{q}(I) \rightarrow \ell_{w}^{q}(I)} \leq\|N\|_{\text {Schur }}$.

Let $f \in \mathcal{S}_{\mathcal{O}}\left(\mathbb{R}^{d}\right)$ be arbitrary. For any $\ell \in I$, define $c_{\ell}:=\left\|\varphi_{\ell}^{*} \cdot \widehat{f}\right\|_{\mathcal{F} L^{p}}$ and $\theta_{\ell}:=\left\|\varphi_{\ell} \cdot \widehat{f}\right\|_{\mathcal{F} L^{p}}$, where $\varphi_{\ell}^{*}:=\sum_{i \in \ell^{*}} \varphi_{i}$. Let $c=\left(c_{i}\right)_{i \in I}$ and $\theta=\left(\theta_{i}\right)_{i \in I}$. Then $0 \leq c_{\ell} \leq \sum_{i \in \ell^{*}} \theta_{i}=\left(\Gamma_{\mathcal{Q}} \theta\right)_{\ell}$, and hence $\|c\|_{\ell_{w}^{q}} \leq\left\|\Gamma_{\mathcal{Q}}\right\|_{\ell_{w}^{q} \rightarrow \ell_{w}^{q}} \cdot\|\theta\|_{\ell_{w}^{q}}=$ $\left\|\Gamma_{\mathcal{Q}}\right\|_{\ell_{w}^{q} \rightarrow \ell_{w}^{q}} \cdot\|f\|_{\mathcal{D}\left(\mathcal{Q}, L^{p}, \ell_{w}^{q}\right)}<\infty$.

Since $f \in \mathcal{S}_{\mathcal{O}}\left(\mathbb{R}^{d}\right)$, we have $\widehat{f} \in C_{c}^{\infty}(\mathcal{O})$, and hence $\widehat{f}=\sum_{\ell \in I} \varphi_{\ell} \cdot \widehat{f}=$ $\sum_{\ell \in I} \varphi_{\ell} \varphi_{\ell}^{*} \widehat{f}$, where only finitely many terms of the series do not vanish. Therefore, by the unconditional convergence of the series defining $R f$ (see Corollary 5.3), we see

$$
\varphi_{i} \cdot \widehat{R f}=\varphi_{i} \cdot \sum_{\alpha \in \Lambda \backslash\{0\}} T_{\alpha}\left(t_{\alpha} \cdot \widehat{f}\right)=\sum_{\ell \in I} \sum_{\alpha \in \Lambda \backslash\{0\}} \varphi_{i} \cdot T_{\alpha}\left(t_{\alpha} \cdot \varphi_{\ell} \cdot \varphi_{\ell}^{*} \cdot \widehat{f}\right) .
$$


Hence, for all $i \in I$,

$$
\begin{aligned}
\left\|\varphi_{i} \cdot \widehat{R f}\right\|_{\mathcal{F}_{L^{p}}} & \leq \sum_{\ell \in I} \sum_{\alpha \in \Lambda \backslash\{0\}}\left\|\varphi_{i} \cdot T_{\alpha}\left(t_{\alpha} \cdot \varphi_{\ell} \cdot \varphi_{\ell}^{*} \cdot \widehat{f}\right)\right\|_{\mathcal{F} L^{p}} \\
& \leq \sum_{\ell \in I} \sum_{\alpha \in \Lambda \backslash\{0\}}\left\|\left(T_{-\alpha} \varphi_{i}\right) \cdot t_{\alpha} \cdot \varphi_{\ell}\right\|_{\mathcal{F} L^{1}}\left\|\varphi_{\ell}^{*} \cdot \widehat{f}\right\|_{\mathcal{F} L^{p}}=(\mathbf{N} c)_{i}
\end{aligned}
$$

and thus

$$
\begin{aligned}
& \|R f\|_{\mathcal{D}\left(\mathcal{Q}, L^{p}, \ell_{w}^{q}\right)}=\left\|\left(\left\|\varphi_{i} \cdot \widehat{R f}\right\|_{\mathcal{F} L^{p}}\right)_{i \in I}\right\|_{\ell_{w}^{q}} \\
& \leq\|\mathbf{N} c\|_{\ell_{w}^{q}} \leq\|N\|_{\text {Schur }}\left\|\Gamma_{\mathcal{Q}}\right\|_{\ell_{w}^{q} \rightarrow \ell_{w}^{q}}\|f\|_{\mathcal{D}\left(\mathcal{Q}, L^{p}, \ell_{w}^{q}\right)},
\end{aligned}
$$

as claimed.

Corollary 5.9 Assume that the hypotheses of Proposition 5.8 are satisfied. Furthermore, assume that the function $t_{0}$ defined in (5.2) is continuous on $\mathcal{O}$ and tame (see Proposition 5.7), so that the operator $\Phi_{t_{0}}: \mathcal{D}\left(\mathcal{Q}, L^{p}, \ell_{w}^{q}\right) \rightarrow \mathcal{D}\left(\mathcal{Q}, L^{p}, \ell_{w}^{q}\right)$ is welldefined and bounded. Finally, assume that $\left(T_{\gamma} g_{j}\right)_{j \in J, \gamma \in C_{j} \mathbb{Z}^{d}}$ is $(w, v, \Phi)$-adapted for some weight $v=\left(v_{j}\right)_{j \in J}$.

Define $T_{0}:=\Phi_{t_{0}}$. Then the frame operator $S: \mathcal{D}\left(\mathcal{Q}, L^{p}, \ell_{w}^{q}\right) \rightarrow \mathcal{D}\left(\mathcal{Q}, L^{p}, \ell_{w}^{q}\right)$ is well-defined and bounded and satisfies $S=T_{0}+R_{0}$ with a bounded linear operator $R_{0}: \mathcal{D}\left(\mathcal{Q}, L^{p}, \ell_{w}^{q}\right) \rightarrow \mathcal{D}\left(\mathcal{Q}, L^{p}, \ell_{w}^{q}\right)$ satisfying

$$
\left\|R_{0}\right\|_{\mathcal{D}\left(\mathcal{Q}, L^{p}, \ell_{w}^{q}\right) \rightarrow \mathcal{D}\left(\mathcal{Q}, L^{p}, \ell_{w}^{q}\right)} \leq C_{p, q}\|N\|_{\text {Schur }}\left\|\Gamma_{\mathcal{Q}}\right\|_{\ell_{w}^{q}(I) \rightarrow \ell_{w}^{q}(I)},
$$

where $N \in \mathbb{C}^{I \times I}$ is as in (5.12), and $C_{p, q}:=1$ if $\max \{p, q\}<\infty$ and $C_{p, q}:=$ $C_{\Phi}\left\|\Gamma_{\mathcal{Q}}\right\|_{\ell_{w}^{q} \rightarrow \ell_{w}^{q}}^{2}$ otherwise.

Proof Corollary 4.10 shows that the frame operator $S: \mathcal{D}\left(\mathcal{Q}, L^{p}, \ell_{w}^{q}\right) \rightarrow$ $\mathcal{D}\left(\mathcal{Q}, L^{p}, \ell_{w}^{q}\right)$ is well-defined and bounded, and hence so is $R_{0}:=S-T_{0}$. Note for $f \in \mathcal{S}_{\mathcal{O}}\left(\mathbb{R}^{d}\right)$ that $T_{0} f=\mathcal{F}^{-1}\left(t_{0} \cdot \widehat{f}\right)$ by Proposition 5.7(iii). Therefore, Corollary 5.3 shows for $f \in \mathcal{S}_{\mathcal{O}}\left(\mathbb{R}^{d}\right)$ that $R_{0} f=R f$ with $R f$ as in Eq. (5.9). Thus, if $\max \{p, q\}<\infty$, the density of $\mathcal{S}_{\mathcal{O}}\left(\mathbb{R}^{d}\right)$ in $\mathcal{D}\left(\mathcal{Q}, L^{p}, \ell_{w}^{q}\right)$ (Proposition 3.13), combined with Proposition 5.8, shows the claim.

Now, suppose that $\max \{p, q\}=\infty$, and let $f \in \mathcal{D}\left(\mathcal{Q}, L^{p}, \ell_{w}^{q}\right)$ be arbitrary. Then, Proposition 3.13 yields a sequence $\left(g_{n}\right)_{n \in \mathbb{N}} \subset \mathcal{S}_{\mathcal{O}}\left(\mathbb{R}^{d}\right)$ and some $F \in \ell_{w}^{q}\left(I ; L^{p}\right)$ such that $g_{n} \rightarrow f$ with convergence in $Z^{\prime}(\mathcal{O})$, and such that each $g_{n}$ is $(F, \Phi)$ dominated, where $\|F\|_{\ell_{w}^{q}\left(I ; L^{p}\right)} \leq C_{p, q} \cdot\|f\|_{\mathcal{D}\left(\mathcal{Q}, L^{p}, \ell_{w}^{q}\right)}$ with $C_{p, q}$ as in the statement of the current corollary. By Proposition 5.7(ii), we get $T_{0} g_{n} \rightarrow T_{0} f$ with convergence in $Z^{\prime}(\mathcal{O})$. In addition, Corollary 4.10 shows that $S g_{n} \rightarrow S f$ in $Z^{\prime}(\mathcal{O})$. Therefore, $R g_{n}=R_{0} g_{n}=\left(S-T_{0}\right) g_{n} \rightarrow\left(S-T_{0}\right) f=R_{0} f$, while Proposition 5.8 shows

$$
\begin{aligned}
\left\|R g_{n}\right\|_{\mathcal{D}\left(\mathcal{Q}, L^{p}, \ell_{w}^{q}\right)} & \leq\|N\|_{\text {Schur }}\left\|\Gamma_{\mathcal{Q}}\right\|_{\ell_{w}^{q} \rightarrow \ell_{w}^{q}}\left\|g_{n}\right\|_{\mathcal{D}\left(\mathcal{Q}, L^{p}, \ell_{w}^{q}\right)} \\
& \leq C_{p, q}\|N\|_{\text {Schur }}\left\|\Gamma_{\mathcal{Q}}\right\|_{\ell_{w}^{q} \rightarrow \ell_{w}^{q}}\|f\|_{\mathcal{D}\left(\mathcal{Q}, L^{p}, \ell_{w}^{q}\right)}
\end{aligned}
$$


Lemma 3.15 yields $\left\|R_{0} f\right\|_{\mathcal{D}\left(\mathcal{Q}, L^{p}, \ell_{w}^{q}\right)} \leq C_{p, q}\|N\|_{\text {Schur }}\left\|\Gamma_{\mathcal{Q}}\right\|_{\ell_{w}^{q} \rightarrow \ell_{w}^{q}}\|f\|_{\mathcal{D}\left(\mathcal{Q}, L^{p}, \ell_{w}^{q}\right)}$.

In many cases, instead of verifying that the matrix $N$ defined in Eq. (5.12) is of Schur-type, it is easier to consider the matrix $\widetilde{N}$ defined next.

Corollary 5.10 Let $\mathcal{Q}=\left(Q_{i}\right)_{i \in I}$ be a decomposition cover of an open set $\mathcal{O} \subset \widehat{\mathbb{R}}^{d}$ of full measure with BAPU $\Phi=\left(\varphi_{i}\right)_{i \in I}$, and let $w=\left(w_{i}\right)_{i \in I}$ be $\mathcal{Q}$-moderate. Let $\left(T_{\gamma} g_{j}\right)_{j \in J, \gamma \in C_{j} \mathbb{Z}^{d}}$ be a generalized shift-invariant system. Suppose that the matrix $\widetilde{N}=\left(\widetilde{N}_{i, \ell}\right)_{i, \ell \in I}$ given by

$$
\widetilde{N}_{i, \ell}:=\max \left\{1, \frac{w_{i}}{w_{\ell}}\right\} \sum_{j \in J} \frac{1}{\left|\operatorname{det} C_{j}\right|} \sum_{\alpha \in C_{j}^{-t} \mathbb{Z}^{d} \backslash\{0\}}\left\|\mathcal{F}^{-1}\left(\varphi_{i}(\cdot-\alpha) \cdot \widehat{g_{j}} \cdot \widehat{g_{j}}(\cdot-\alpha) \cdot \varphi_{\ell}\right)\right\|_{L^{1}}
$$

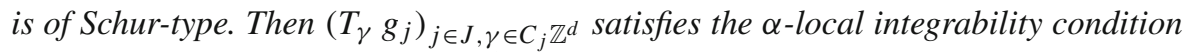
relative to $\mathcal{O}^{c}$, and $\|N\|_{\text {Schur }} \leq\|\widetilde{N}\|_{\text {Schur }}$, where $N$ is as defined in $E q$. (5.12).

Proof By assumption, $\|\tilde{N}\|_{\text {Schur }}<\infty$. We first show that

$$
C:=\underset{\xi \in \mathcal{O}}{\operatorname{ess} \sup } \sum_{j \in J} \frac{1}{\left|\operatorname{det} C_{j}\right|} \sum_{\alpha \in C_{j}^{-t} \mathbb{Z}^{d}}\left|\widehat{g_{j}}(\xi) \widehat{g_{j}}(\xi+\alpha)\right|<\infty .
$$

To show this, first note that since $\mathcal{O} \subset \widehat{\mathbb{R}}^{d}$ is of full measure, so is

$$
\mathcal{O}_{0}:=\left\{\xi \in \widehat{\mathbb{R}}^{d}: \quad \xi+\alpha \in \mathcal{O}, \quad \forall j \in J, \forall \alpha \in C_{j}^{-t} \mathbb{Z}^{d}\right\},
$$

since $\mathcal{O}_{0}^{c}=\bigcup_{j \in J} \bigcup_{\alpha \in C_{j}^{-t} \mathbb{Z}^{d}}\left(\mathcal{O}^{c}-\alpha\right)$ is a countable union of null-sets. If $\xi \in \mathcal{O}_{0}$ and $j \in J, \alpha \in C_{j}^{-t} \mathbb{Z}^{d}$ are arbitrary, then $\xi+\alpha \in \mathcal{O}$ and hence $\sum_{i \in I} \varphi_{i}(\xi+\alpha)=1$, whence $1 \leq \sum_{i \in I}\left|\varphi_{i}(\xi+\alpha)\right|$. Now, let $\xi \in \mathcal{O}_{0} \subset \mathcal{O}$ be arbitrary and choose $i_{0} \in I$ such that $\xi \in Q_{i_{0}}$. Then, $\sum_{\ell \in i_{0}^{*}} \varphi_{\ell}(\xi)=1$. Thus, using the estimate $\|f\|_{\text {sup }} \leq$ $\left\|\mathcal{F}^{-1} f\right\|_{L^{1}}$, we see that

$$
\begin{aligned}
& \sum_{j \in J} \frac{1}{\left|\operatorname{det} C_{j}\right|} \sum_{\alpha \in C_{j}^{-t} \mathbb{Z}^{d} \backslash\{0\}}\left|\widehat{g_{j}}(\xi) \widehat{g_{j}}(\xi+\alpha)\right| \\
& \quad \leq \sum_{i \in I, \ell \in i_{0}^{*}} \sum_{j \in J} \frac{1}{\left|\operatorname{det} C_{j}\right|} \sum_{\alpha \in C_{j}^{-t} \mathbb{Z}^{d} \backslash\{0\}}\left|\widehat{g_{j}}(\xi) \varphi_{i}(\xi+\alpha) \widehat{g_{j}}(\xi+\alpha) \varphi_{\ell}(\xi)\right| \\
& \quad \leq \sum_{\ell \in i_{0}^{*}, i \in I} \tilde{N}_{i, \ell} \leq N_{\mathcal{Q}} \cdot\|\tilde{N}\|_{\text {Schur }}<\infty .
\end{aligned}
$$

In combination with our standing assumption (4.1), this proves (5.14). 
Now, the monotone convergence theorem and (5.14) show for arbitrary $f \in$ $\mathcal{B}_{\mathcal{O}}\left(\mathbb{R}^{d}\right)$ that

$$
\begin{array}{r}
\sum_{j \in J} \frac{1}{\left|\operatorname{det} C_{j}\right|} \sum_{\alpha \in C_{j}^{-t} \mathbb{Z}^{d}} \int_{\widehat{\mathbb{R}}^{d}}\left|\widehat{f}(\xi) \widehat{f}(\xi+\alpha) \widehat{g_{j}}(\xi) \widehat{g_{j}}(\xi+\alpha)\right| d \xi \\
\leq C\|\widehat{f}\|_{L^{\infty}} \cdot \int_{\widehat{\mathbb{R}}^{d}}|\widehat{f}(\xi)| d \xi<\infty
\end{array}
$$

since $\widehat{f} \in L^{\infty}\left(\widehat{\mathbb{R}}^{d}\right)$ and supp $\widehat{f} \subset \mathcal{O}$ is compact. This shows that $\left(T_{\gamma} g_{j}\right)_{j \in J, \gamma \in C_{j} \mathbb{Z}^{d}}$ satisfies the $\alpha$-LIC.

Finally, recall that $t_{\alpha}(\xi)=\sum_{j \in \kappa(\alpha)}\left|\operatorname{det} C_{j}\right|^{-1} \widehat{g_{j}}(\xi) \widehat{g_{j}}(\xi+\alpha)$, where $\kappa(\alpha)=$ $\left\{j \in J: \alpha \in C_{j}^{-t} \mathbb{Z}^{d}\right\}$. Therefore, the matrix entries $N_{i, \ell}$ defined in (5.12) satisfy

$$
\begin{array}{r}
N_{i, \ell} \leq \max \left\{1, \frac{w_{i}}{w_{\ell}}\right\} \sum_{\alpha \in \Lambda \backslash\{0\}} \sum_{j \in \kappa(\alpha)}\left|\operatorname{det} C_{j}\right|^{-1}\left\|\mathcal{F}^{-1}\left(\varphi_{i}(\cdot+\alpha) \cdot \widehat{g_{j}} \cdot \widehat{g_{j}}(\cdot+\alpha) \cdot \varphi_{\ell}\right)\right\|_{L^{1}} \\
=\widetilde{N}_{i, \ell}
\end{array}
$$

Thus, $\|N\|_{\text {Schur }} \leq\|\tilde{N}\|_{\text {Schur }}$, as claimed.

\subsection{Invertibility in the Case $(p, q)=(2,2)$}

In this subsection, we focus on the special case $(p, q)=(2,2)$, where the following identification holds; see [61,Lemma 6.10].

Lemma 5.11 Let $\mathcal{Q}=\left(Q_{i}\right)_{i \in I}$ be a decomposition cover of an open set $\emptyset \neq \mathcal{O} \subset \widehat{\mathbb{R}}^{d}$, and let $w=\left(w_{i}\right)_{i \in I}$ be a $\mathcal{Q}$-moderate weight. Then there is a measurable weight $v$ : $\mathcal{O} \rightarrow(0, \infty)$ with $v(\xi) \asymp w_{i}$ for all $\xi \in Q_{i}$ and $i \in I$. Furthermore, $\mathcal{D}\left(\mathcal{Q}, L^{2}, \ell_{w}^{2}\right)=$ $\mathcal{F}^{-1}\left(L_{v}^{2}(\mathcal{O})\right)$ with equivalent norms, where the norm $\|f\|_{\mathcal{F}^{-1}\left(L_{v}^{2}(\mathcal{O})\right)}:=\|\widehat{f}\|_{L_{v}^{2}(\mathcal{O})}$ is used on $\mathcal{F}^{-1}\left(L_{v}^{2}(\mathcal{O})\right)=\left\{f \in Z^{\prime}(\mathcal{O}): \widehat{f} \in L_{v}^{2}(\mathcal{O})\right\}$.

We will also make use of the following two lemmata.

Lemma 5.12 Let $\emptyset \neq \mathcal{O} \subset \widehat{\mathbb{R}}^{d}$ be an open set, let $v: \mathcal{O} \rightarrow(0, \infty)$ be a weight function, and let $t_{0}$ be as in Eq. (5.2). Then, the Fourier multipliers $T_{0}: \mathcal{F}^{-1}\left(L_{v}^{2}(\mathcal{O})\right) \rightarrow$ $\mathcal{F}^{-1}\left(L_{v}^{2}(\mathcal{O})\right), f \mapsto \mathcal{F}^{-1}\left(t_{0} \widehat{f}\right)$ and

$$
T_{0}^{-1}: \mathcal{F}^{-1}\left(L_{v}^{2}(\mathcal{O})\right) \rightarrow \mathcal{F}^{-1}\left(L_{v}^{2}(\mathcal{O})\right), \quad f \mapsto \mathcal{F}^{-1}\left(t_{0}^{-1} \cdot \widehat{f}\right)
$$

are well-defined and bounded, with $\left\|T_{0}^{-1}\right\|_{\mathrm{op}} \leq A^{-1}$ and $\left\|T_{0}\right\|_{\mathrm{op}} \leq B$, where $A, B>0$ are as in (4.1).

Proof If $f \in \mathcal{F}^{-1}\left(L_{v}^{2}(\mathcal{O})\right)$, then

$$
\left\|T_{0}^{-1} f\right\|_{\mathcal{F}^{-1}\left(L_{v}^{2}(\mathcal{O})\right)}=\left\|t_{0}^{-1} \cdot \widehat{f}\right\|_{L_{v}^{2}(\mathcal{O})} \leq\left\|t_{0}^{-1}\right\|_{L^{\infty}(\mathcal{O})} \cdot\|f\|_{\mathcal{F}^{-1}\left(L_{v}^{2}(\mathcal{O})\right)} .
$$

The argument for $T_{0}$ is similar. 
Lemma 5.13 Let $\mathcal{O} \subset \widehat{\mathbb{R}}^{d}$ be an open set of full measure and let $v: \widehat{\mathbb{R}}^{d} \rightarrow(0, \infty)$ be $v_{0}$-moderate for some symmetric weight $v_{0}: \widehat{\mathbb{R}}^{d} \rightarrow(0, \infty)$; that is, $v(\xi+\eta) \leq$ $C_{v} \cdot v(\xi) \cdot v_{0}(\eta)$ for all $\xi, \eta \in \widehat{\mathbb{R}}^{d}$ and some $C_{v}>0$. Then the operator $R$ defined in Eq. (5.9) satisfies

$$
\|R\|_{\mathcal{F}^{-1}\left(L_{v}^{2}(\mathcal{O})\right) \rightarrow \mathcal{F}^{-1}\left(L_{v}^{2}(\mathcal{O})\right)} \leq C_{v} \cdot \underset{\xi \in \mathcal{O}}{\operatorname{ess} \sup } \sum_{\alpha \in \Lambda \backslash\{0\}}\left|t_{\alpha}(\xi)\right| \cdot v_{0}(\alpha) .
$$

Proof Since $\mathcal{O}$ is of full measure, we have $\mathcal{F}^{-1}\left(L_{v}^{2}(\mathcal{O})\right)=\mathcal{F}^{-1}\left(L_{v}^{2}\left(\widehat{\mathbb{R}}^{d}\right)\right)$, up to canonical identifications. Let $g \in L^{2}\left(\widehat{\mathbb{R}}^{d}\right)$ and $f \in \mathcal{F}^{-1}\left(L_{v}^{2}(\mathcal{O})\right)$ be such that $\|g\|_{L^{2}} \leq$ 1 and $\|f\|_{\mathcal{F}^{-1}\left(L_{v}^{2}(\mathcal{O})\right)} \leq 1$. Using the estimates $v(\xi) \leq C_{v} \cdot v(\xi-\alpha) \cdot v_{0}(\alpha)$ and $|a b| \leq \frac{1}{2}\left(|a|^{2}+|b|^{2}\right)$ and the identity $t_{\alpha}(\xi-\alpha)=\overline{t_{-\alpha}(\xi)}$, it follows that

$$
\begin{aligned}
& \int_{\widehat{\mathbb{R}}^{d}}|g(\xi)| \cdot v(\xi) \cdot \sum_{\alpha \in \Lambda \backslash\{0\}}\left|t_{\alpha}(\xi-\alpha) \widehat{f}(\xi-\alpha)\right| d \xi \\
& \leq C_{v} \cdot \sum_{\alpha \in \Lambda \backslash\{0\}} v_{0}(\alpha) \int_{\widehat{\mathbb{R}}^{d}}\left(\left|t_{-\alpha}(\xi)\right|^{1 / 2} \cdot|g(\xi)|\right) \cdot\left(\left|t_{\alpha}(\xi-\alpha)\right|^{1 / 2} \cdot|(v \widehat{f})(\xi-\alpha)|\right) d \xi \\
& \leq \frac{C_{v}}{2} \cdot \sum_{\alpha \in \Lambda \backslash\{0\}} v_{0}(\alpha) \int_{\widehat{\mathbb{R}}^{d}}\left|t_{-\alpha}(\xi)\right| \cdot|g(\xi)|^{2}+\left|t_{\alpha}(\xi-\alpha)\right| \cdot|(v \widehat{f})(\xi-\alpha)|^{2} d \xi \\
& =\frac{C_{v}}{2} \cdot\left(\int_{\widehat{\mathbb{R}}^{d}}\left(\sum_{\beta \in \Lambda \backslash\{0\}} v_{0}(-\beta)\left|t_{\beta}(\xi)\right|\right) \cdot|g(\xi)|^{2} d \xi\right. \\
& \left.+\int_{\widehat{\mathbb{R}}^{d}}\left(\sum_{\alpha \in \Lambda \backslash\{0\}} v_{0}(\alpha)\left|t_{\alpha}(\eta)\right|\right) \cdot|(v \widehat{f})(\eta)|^{2} d \eta\right) \\
& \leq C_{v} \cdot \underset{\xi \in \mathcal{O}}{\operatorname{ess} \sup _{\alpha \in \Lambda \backslash\{0\}} v_{0}(\alpha)\left|t_{\alpha}(\xi)\right| .}
\end{aligned}
$$

Since this holds for all $g \in L^{2}\left(\widehat{\mathbb{R}}^{d}\right)$ with $\|g\|_{L^{2}} \leq 1$, the series

$$
\sum_{\alpha \in \Lambda \backslash\{0\}} t_{\alpha}(\xi-\alpha) \widehat{f}(\xi-\alpha)=\sum_{\alpha \in \Lambda \backslash\{0\}}\left[T_{\alpha}\left(t_{\alpha} \cdot \widehat{f}\right)\right](\xi)=[\widehat{R f}](\xi)
$$

is almost everywhere absolutely convergent, and

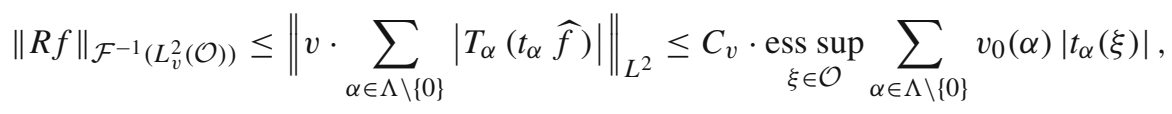

for all $f \in \mathcal{F}^{-1}\left(L_{v}^{2}(\mathcal{O})\right)$ with $\|f\|_{\mathcal{F}^{-1}\left(L_{v}^{2}(\mathcal{O})\right)} \leq 1$. This proves the claim.

Using the previous lemmata, the following result follows easily. See [45, Theorem 3.3] for a similar result in $L^{2}$. 
Proposition 5.14 Let $\mathcal{Q}=\left(Q_{i}\right)_{i \in I}$ be a decomposition cover of an open set $\mathcal{O} \subset \widehat{\mathbb{R}}^{d}$ of full measure, and let $w=\left(w_{i}\right)_{i \in I}$ be $\mathcal{Q}$-moderate. Suppose $\left(T_{\gamma} g_{j}\right)_{j \in J, \gamma \in C_{j} \mathbb{Z}^{d}}$ satisfies the $\alpha$-local integrability condition (5.1) relative to $\mathcal{O}^{c}$. Finally, assume that

$$
C_{v} \cdot \underset{\xi \in \mathcal{O}}{\operatorname{ess} \sup } \sum_{\alpha \in \Lambda \backslash\{0\}}\left|t_{\alpha}(\xi)\right| \cdot v_{0}(\alpha)<A,
$$

where $A>0$ is as in (4.1), where $v: \widehat{\mathbb{R}}^{d} \rightarrow(0, \infty)$ is a measurable weight that satisfies $v(\xi) \asymp w_{i}$ for all $\xi \in Q_{i}$ and $i \in I$, and where $v_{0}: \widehat{\mathbb{R}}^{d} \rightarrow(0, \infty)$ is assumed to be a symmetric weight satisfying $v(\xi+\eta) \leq C_{v} \cdot v(\xi) \cdot v_{0}(\eta)$ for all $\xi, \eta \in \widehat{\mathbb{R}}^{d}$.

Then, the frame operator $S: \mathcal{S}_{\mathcal{O}}\left(\mathbb{R}^{d}\right) \rightarrow L^{2}\left(\mathbb{R}^{d}\right)$ associated to $\left(T_{\gamma} g_{j}\right)_{j \in J, \gamma \in C_{j} \mathbb{Z}^{d}}$ uniquely extends to a bounded linear operator $S_{0}: \mathcal{D}\left(\mathcal{Q}, L^{2}, \ell_{w}^{2}\right) \rightarrow \mathcal{D}\left(\mathcal{Q}, L^{2}, \ell_{w}^{2}\right)$. This extended operator is boundedly invertible.

Proof Lemmas 5.12 and 5.13 show, respectively, that the operators $T_{0}$ and $R$ defined in these lemmas yield bounded operators on $\mathcal{F}^{-1}\left(L_{v}^{2}(\mathcal{O})\right)$, so that $S_{0}:=T_{0}+R$ : $\mathcal{F}^{-1}\left(L_{v}^{2}(\mathcal{O})\right) \rightarrow \mathcal{F}^{-1}\left(L_{v}^{2}(\mathcal{O})\right)$ is well-defined and bounded. As seen in Proposition 5.2, we have $S_{0} f=S f$ for all $f \in \mathcal{S}_{\mathcal{O}}\left(\mathbb{R}^{d}\right) \subset \mathcal{B}_{\mathcal{O}}\left(\mathbb{R}^{d}\right)$. Furthermore, $\mathcal{S}_{\mathcal{O}}\left(\mathbb{R}^{d}\right) \subset$ $\mathcal{D}\left(\mathcal{Q}, L^{2}, \ell_{w}^{2}\right)=\mathcal{F}^{-1}\left(L_{v}^{2}(\mathcal{O})\right)$ is dense (see Proposition 3.13 and Lemma 5.11); therefore, $S_{0}$ is the unique bounded extension of $S$.

Finally, conditions (4.1) and (5.16) together with Lemma 5.12 and Lemma 5.13 yield that

$$
\left\|T_{0}^{-1}\right\|_{\mathcal{F}^{-1}\left(L_{v}^{2}(\mathcal{O})\right) \rightarrow \mathcal{F}^{-1}\left(L_{v}^{2}(\mathcal{O})\right)} \cdot\|R\|_{\mathcal{F}^{-1}\left(L_{v}^{2}(\mathcal{O})\right) \rightarrow \mathcal{F}^{-1}\left(L_{v}^{2}(\mathcal{O})\right)}<1
$$

Hence, $S_{0}=T_{0}+R$ is boundedly invertible on $\mathcal{F}^{-1}\left(L_{v}^{2}(\mathcal{O})\right)$ by Lemma 5.4. Using the norm equivalence $\|\cdot\|_{\mathcal{F}^{-1}\left(L_{v}^{2}(\mathcal{O})\right)} \asymp\|\cdot\|_{\mathcal{D}\left(\mathcal{Q}, L^{2}, \ell_{w}^{2}\right)}$ provided by Lemma 5.11 , it follows therefore that also $S_{0}: \mathcal{D}\left(\mathcal{Q}, L^{2}, \ell_{w}^{2}\right) \rightarrow \mathcal{D}\left(\mathcal{Q}, L^{2}, \ell_{w}^{2}\right)$ is boundedly invertible.

Remark 5.15 The formulation of Proposition 5.14 is rather technical, because, under those assumptions, the formula defining the frame operator might not make sense for $f \in \mathcal{D}\left(\mathcal{Q}, L^{2}, \ell_{w}^{2}\right)$. Indeed, the hypothesis are satisfied for every tight frame, even if $g_{j} \notin \mathcal{D}\left(\mathcal{Q}, L^{2}, \ell_{w}^{2}\right)$. If, in addition, $\left(T_{\gamma} g_{j}\right)_{j \in J, \gamma \in C_{j} \mathbb{Z}^{d}}$ is assumed to be $(w, v, \Phi)$-adapted for some weight $v$, then Proposition 4.8 applies and we can conclude unambiguously that $S: \mathcal{D}\left(\mathcal{Q}, L^{2}, \ell_{w}^{2}\right) \rightarrow \mathcal{D}\left(\mathcal{Q}, L^{2}, \ell_{w}^{2}\right)$ is well-defined, bounded and boundedly invertible on $\mathcal{D}\left(\mathcal{Q}, L^{2}, \ell_{w}^{2}\right)$.

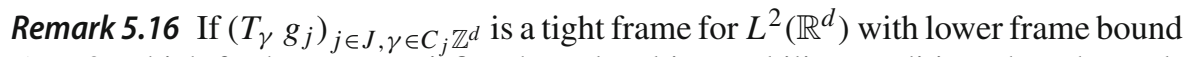
$A>0$, which furthermore satisfies the $\alpha$-local integrability condition, then the multipliers $t_{\alpha} \in L^{\infty}\left(\widehat{\mathbb{R}}^{d}\right)$ satisfy $t_{\alpha}(\xi)=A \delta_{\alpha, 0}$ for a.e. $\xi \in \widehat{\mathbb{R}}^{d}$ and all $\alpha \in \Lambda$, cf. [41,Theorem 3.4]. The condition (5.16) is then obviously satisfied. The placement of the absolute value sign outside of the series defining the multipliers $t_{\alpha}$ allows for cancellations, which can be very important [45]. 


\section{Concrete Estimates for Affinely Generated Covers}

In this section, we simplify the results of Sect. 5 for the case that the decomposition cover $\mathcal{Q}$ is affinely generated. The results obtained here will be further simplified in Sect. 7.

In the sequel, we will repeatedly use $\mathcal{Q}$-localized versions of the generating

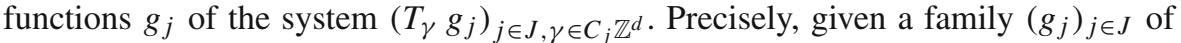
generating functions $g_{j} \in L^{1}\left(\mathbb{R}^{d}\right) \cap L^{2}\left(\mathbb{R}^{d}\right)$ and a family $\left(S_{i}\right)_{i \in I}$ of invertible affinelinear maps $S_{i}=A_{i}(\cdot)+b_{i}$, we let

$g_{i, j}^{\natural}:=\left|\operatorname{det} A_{i}\right|^{-1} \cdot\left(M_{-b_{i}} g_{j}\right) \circ A_{i}^{-t}=\mathcal{F}^{-1}\left(\widehat{g_{j}} \circ S_{i}\right)$ for $(i, j) \in I \times J$,

so that $\mathcal{F} g_{i, j}^{\natural}=\widehat{g_{j}} \circ S_{i}$.

\subsection{Boundedness of the Frame Operator}

As a first step, we provide a sufficient condition for a system to be adapted (see Definition 4.3). The proof makes use of the following self-improving property of amalgam spaces, which is taken from [62,Theorem 2.17].

Lemma 6.1 Let $f \in \mathcal{S}^{\prime}\left(\mathbb{R}^{d}\right)$ with supp $\widehat{f} \subset A[-R, R]^{d}+\xi_{0}$ for some $A \in \operatorname{GL}(d, \mathbb{R})$, $\xi_{0} \in \widehat{\mathbb{R}}^{d}$, and $R>0$. Then, there exists a constant $C=C(d)>0$ which only depends on $d \in \mathbb{N}$ such that

$$
\|f\|_{W_{A^{-t}[-1,1]}^{d}}\left(L^{\infty}, L^{1}\right) \leq C \cdot(1+R)^{d} \cdot\|f\|_{L^{1}} .
$$

Proposition 6.2 Let $\mathcal{Q}=\left(A_{i}\left(Q_{i}^{\prime}\right)+b_{i}\right)_{i \in I}$ be an affinely generated cover of $\mathcal{O} \subset \widehat{\mathbb{R}}^{d}$, and let $\Phi=\left(\varphi_{i}\right)_{i \in I}$ be a regular partition of unity subordinate to $\mathcal{Q}$. Let $w=$ $\left(w_{i}\right)_{i \in I}$ be $\mathcal{Q}$-moderate, and let $v=\left(v_{j}\right)_{j \in J}$ be a weight. Suppose that the system

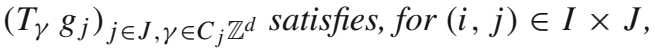

$$
G_{i, j}:=\max \left\{\frac{w_{i}}{v_{j}}, \frac{v_{j}}{w_{i}}\right\} \frac{\left(1+\left\|C_{j}^{t} A_{i}\right\|\right)^{d}}{\left|\operatorname{det} C_{j}\right|^{1 / 2}} \int_{Q_{i}^{\prime}} \max _{|\theta| \leq d+1}\left|\partial^{\theta}\left[\mathcal{F} g_{i, j}^{\natural}\right](\xi)\right| d \xi<\infty
$$

and that $G=\left(G_{i, j}\right)_{i \in I, j \in J} \in \mathbb{C}^{I \times J}$ is of Schur-type. Then, $\left(T_{\gamma} g_{j}\right)_{j \in J, \gamma \in C_{j} \mathbb{Z}^{d}}$ is $(w, v, \Phi)$-adapted. Consequently, the frame operator $S: \mathcal{D}\left(\mathcal{Q}, L^{p}, \ell_{w}^{q}\right) \rightarrow$ $\mathcal{D}\left(\mathcal{Q}, L^{p}, \ell_{w}^{q}\right)$ is well-defined and bounded.

Proof We will estimate $\left\|\left(\check{\varphi}_{i} * g_{j}\right) \circ C_{j}\right\|_{W\left(C_{0}, \ell^{1}\right)}$ for $(i, j) \in I \times J$. Choose $r>1$ such that $\overline{Q_{i}^{\prime}} \subset[-r, r]^{d}$ for all $i \in I$. The norm equivalence $\|\cdot\|_{W\left(C_{0}, \ell^{1}\right)} \asymp \|$. 
$\|_{W_{[-1,1]^{d}}\left(C_{0}, L^{1}\right)}$ yields an absolute constant $K_{1}=K_{1}(d)>0$ satisfying

$$
\begin{aligned}
\left\|\left(\check{\varphi}_{i} * g_{j}\right) \circ C_{j}\right\|_{W\left(C_{0}, \ell^{1}\right)} & \leq K_{1} \cdot\left\|\left(\check{\varphi}_{i} * g_{j}\right) \circ C_{j}\right\|_{W_{[-1,1]}^{d}\left(C_{0}, L^{1}\right)} \\
& =K_{1} \cdot\left|\operatorname{det} C_{j}\right|^{-1} \cdot\left\|\breve{\varphi}_{i} * g_{j}\right\|_{W_{C_{j}\left([-1,1]^{d}\right)}\left(C_{0}, L^{1}\right)}
\end{aligned}
$$

for $i \in I$ and $j \in J$. Here, we used Eq. (2.1) in the last step. Define $P_{i, j}:=r$. $\left\|C_{j}^{t} A_{i}\right\|_{\ell^{\infty} \rightarrow \ell^{\infty}}$. Since supp $\varphi_{i} \subset A_{i}\left(\overline{Q_{i}^{\prime}}\right)+b_{i}$, it follows that

$$
\begin{aligned}
& \operatorname{supp} \mathcal{F}\left(\breve{\varphi}_{i} * g_{j}\right) \subset A_{i}[-r, r]^{d}+b_{i}=C_{j}^{-t}\left(C_{j}^{t} \cdot A_{i}[-r, r]^{d}\right) \\
& \quad+b_{i} \subset C_{j}^{-t}\left[-P_{i, j}, P_{i, j}\right]^{d}+b_{i} .
\end{aligned}
$$

Therefore, Lemma 6.1 yields a constant $K_{2}=K_{2}(d)>0$ such that

$$
\left\|\left(\check{\varphi}_{i} * g_{j}\right) \circ C_{j}\right\|_{W\left(C_{0}, \ell^{1}\right)} \leq K_{1} K_{2} \cdot\left(1+P_{i, j}\right)^{d} \cdot\left|\operatorname{det} C_{j}\right|^{-1} \cdot\left\|\mathcal{F}^{-1}\left(\varphi_{i} \cdot \widehat{g_{j}}\right)\right\|_{L^{1}}
$$

Next, recalling the notion of the normalized version $\varphi_{i}^{\mathrm{b}}=\varphi_{i} \circ S_{i}$ of $\varphi_{i}$ (Definition 3.6), we see

$$
\left\|\mathcal{F}^{-1}\left(\varphi_{i} \cdot \widehat{g_{j}}\right)\right\|_{L^{1}}=\left\|\mathcal{F}^{-1}\left(\left(\varphi_{i} \circ S_{i}\right) \cdot\left(\widehat{g_{j}} \circ S_{i}\right)\right)\right\|_{L^{1}}=\left\|\mathcal{F}^{-1}\left(\varphi_{i}^{b} \cdot \mathcal{F} g_{i, j}^{\natural}\right)\right\|_{L^{1}},
$$

whence Lemma A.2 shows that

$$
\left\|\mathcal{F}^{-1}\left(\varphi_{i} \cdot \widehat{g_{j}}\right)\right\|_{L^{1}} \leq \frac{d+1}{\pi^{d}} \max _{|\theta| \leq d+1}\left\|\partial^{\theta}\left(\varphi_{i}^{b} \cdot \mathcal{F} g_{i, j}^{\natural}\right)\right\|_{L^{1}} \cdot
$$

Now, since $\varphi_{i}^{b}$ vanishes outside of $Q_{i}^{\prime}$, it follows that $\left|\left(\partial^{\alpha} \varphi_{i}^{b}\right)(\xi)\right| \leq K_{3} \cdot \mathbb{1}_{Q_{i}^{\prime}}(\xi)$ for all $\xi \in \widehat{\mathbb{R}}^{d}$ and any $\alpha \in \mathbb{N}_{0}^{d}$ with $|\alpha| \leq d+1$, where $K_{3}:=\max _{|\alpha| \leq d+1} \sup _{i \in I}\left\|\partial^{\alpha} \varphi_{i}^{b}\right\|_{L^{\infty}}$. An application of the Leibniz rule therefore yields

$$
\begin{aligned}
\left|\partial^{\theta}\left(\varphi_{i}^{b} \cdot \mathcal{F} g_{i, j}^{\natural}\right)(\xi)\right| & \leq \sum_{\beta \leq \theta}\left(\begin{array}{c}
\theta \\
\beta
\end{array}\right)\left|\left(\partial^{\theta-\beta} \varphi_{i}^{b}\right)(\xi)\right| \cdot\left|\partial^{\beta}\left[\mathcal{F} g_{i, j}^{\natural}\right](\xi)\right| \\
& \leq 2^{d+1} K_{3} \cdot \mathbb{1}_{Q_{i}^{\prime}}(\xi) \max _{|\nu| \leq d+1}\left|\left(\partial^{v}\left[\mathcal{F} g_{i, j}^{\natural}\right]\right)(\xi)\right|
\end{aligned}
$$

for any $\theta \in \mathbb{N}_{0}^{d}$ with $|\theta| \leq d+1$. Integrating this last inequality and combining it with (6.2) yields

$$
\left\|\left(\breve{\varphi}_{i} * g_{j}\right) \circ C_{j}\right\|_{W\left(C_{0}, \ell^{1}\right)} \leq K \frac{\left(1+\left\|C_{j}^{t} A_{i}\right\|\right)^{d}}{\left|\operatorname{det} C_{j}\right|} \int_{Q_{i}^{\prime}} \max _{|\theta| \leq d+1}\left|\left(\partial^{\theta}\left[\mathcal{F} g_{i, j}^{\natural}\right]\right)(\xi)\right| d \xi
$$


for a constant $K=K(\mathcal{Q}, d, \Phi)>0$. Therefore, the matrix entries $M_{i, j}$ defined in Eq. (4.2) satisfy

$$
\begin{aligned}
0 \leq M_{i, j} & =\max \left\{\frac{w_{i}}{v_{j}}, \frac{v_{j}}{w_{i}}\right\} \cdot\left|\operatorname{det} C_{j}\right|^{1 / 2} \cdot\left\|\left(\breve{\varphi}_{i} * g_{j}\right) \circ C_{j}\right\|_{W\left(C_{0}, \ell^{1}\right)} \\
& \leq K \cdot \max \left\{\frac{w_{i}}{v_{j}}, \frac{v_{j}}{w_{i}}\right\} \cdot \frac{\left(1+\left\|C_{j}^{t} A_{i}\right\|\right)^{d}}{\left|\operatorname{det} C_{j}\right|^{1 / 2}} \int_{Q_{i}^{\prime}} \max _{|\theta| \leq d+1}\left|\left(\partial^{\theta}\left[\mathcal{F} g_{i, j}^{\natural}\right]\right)(\xi)\right| d \xi
\end{aligned}
$$

$=K \cdot G_{i, j}$.

This implies $\|M\|_{\text {Schur }} \leq K \cdot\|G\|_{\text {Schur }}<\infty$, so that $\left(T_{\gamma} g_{j}\right)_{j \in J, \gamma \in C_{j} \mathbb{Z}^{d}}$ is $(w, v, \Phi)$ adapted.

\subsection{The Main Term}

In this section, we provide a simplified bound for the operator norm of $T_{0}^{-1}$ : $\mathcal{D}\left(\mathcal{Q}, L^{p}, \ell_{w}^{q}\right) \rightarrow \mathcal{D}\left(\mathcal{Q}, L^{p}, \ell_{w}^{q}\right)$.

Proposition 6.3 Let $\mathcal{Q}=\left(S_{i}\left(Q_{i}^{\prime}\right)\right)_{i \in I}$ be an affinely generated cover of an open set $\mathcal{O} \subset \widehat{\mathbb{R}}^{d}$ of full measure. Let $\Phi=\left(\varphi_{i}\right)_{i \in I}$ be a regular partition of unity subordinate to $\mathcal{Q}$. Suppose the system $\left(T_{\gamma} g_{j}\right)_{j \in J, \gamma \in C_{j} \mathbb{Z}^{d} \text { satisfies }}$

$$
M:=\sup _{i \in I} \sum_{j \in J}\left(\left|\operatorname{det} C_{j}\right|^{-1} \cdot\left\|\left.\max _{|\nu| \leq d+1}\left|\partial^{v}\right| \mathcal{F} g_{i, j}^{\natural}\right|^{2} \mid\right\|_{L^{d+1}\left(Q_{i}^{\prime}\right)}\right)<\infty .
$$

Then, the function $t_{0}$ defined in Eq. (5.2) is continuous on $\mathcal{O}$ and tame, and Eq. (4.1) holds for all $\xi \in \mathcal{O}$. Furthermore, for all $p, q \in[1, \infty]$ and any $\mathcal{Q}$-moderate weight $w=\left(w_{i}\right)_{i \in I}$, the operator

$$
T_{0}:=\Phi_{t_{0}}: \mathcal{D}\left(\mathcal{Q}, L^{p}, \ell_{w}^{q}\right) \rightarrow \mathcal{D}\left(\mathcal{Q}, L^{p}, \ell_{w}^{q}\right)
$$

with $\Phi_{t_{0}}$ as in Proposition 5.7 is well-defined, bounded, and boundedly invertible, with

$$
\left\|T_{0}^{-1}\right\|_{\mathcal{D}\left(\mathcal{Q}, L^{p}, \ell_{w}^{q}\right) \rightarrow \mathcal{D}\left(\mathcal{Q}, L^{p}, \ell_{w}^{q}\right)} \leq C_{d} \cdot N_{\mathcal{Q}}^{2} C_{\Phi} \cdot\left[\max _{|\alpha| \leq d+1} C_{\mathcal{Q}, \Phi, \alpha}\right] \cdot A^{-1} \cdot\left(\frac{M}{A}\right)^{d+1}
$$

where $A>0$ is as in (4.1) and

$$
C_{d}:=\frac{3 \cdot(d+1)^{3 / 2} \cdot 2^{d+1}}{\pi^{d}}\left(\frac{\frac{0.8}{e} \cdot(d+1)^{2}}{\ln (2+d)}\right)^{d+1} .
$$

Proof We divide the proof into four steps.

Step 1. We show that the series defining $t_{0}$ converges locally uniformly on $\mathcal{O}$, that Eq. (4.1) holds pointwise on $\mathcal{O}$, and that $t_{0}$ is tame. 
To see this, set $\gamma_{j}:=\left|{\widehat{g_{j}}}^{2} /\right| \operatorname{det} C_{j} \mid$, and note $t_{0}=\sum_{j \in J} \gamma_{j}$ and that $\gamma_{j} \in C^{\infty}\left(\widehat{\mathbb{R}}^{d}\right)$ thanks to our standing assumptions regarding the $g_{j}$. Now, for arbitrary $i \in I$, recall that $\varphi_{i}^{b}=\varphi_{i} \circ S_{i}$ vanishes outside $Q_{i}^{\prime}$, so that the Leibniz rule shows

$$
\begin{aligned}
\left|\partial^{\alpha}\left(\varphi_{i}^{b} \cdot\left(\gamma_{j} \circ S_{i}\right)\right)(\xi)\right| & \leq \sum_{\beta \leq \alpha}\left(\begin{array}{c}
\alpha \\
\beta
\end{array}\right)\left|\partial^{\alpha-\beta} \varphi_{i}^{b}(\xi)\right|\left|\partial^{\beta}\left(\gamma_{j} \circ S_{i}\right)(\xi)\right| \\
& \leq\left. c_{0} \cdot\left|\operatorname{det} C_{j}\right|^{-1} \cdot \mathbb{1}_{Q_{i}^{\prime}}(\xi) \cdot \max _{|\nu| \leq d+1}\left|\partial^{\nu}\right| \mathcal{F} g_{i, j}^{\natural}\right|^{2}(\xi) \mid
\end{aligned}
$$

for $c_{0}:=2^{d+1} \max _{|v| \leq d+1} C_{\mathcal{Q}, \Phi, v}$ and arbitrary $\alpha \in \mathbb{N}_{0}^{d}$ with $|\alpha| \leq d+1$.

Therefore, using the notation $\mathbb{I}:=\{0\} \cup\left\{(d+1) e_{\ell}: \ell \in \underline{d}\right\}$ (where $\left(e_{1}, \ldots, e_{d}\right.$ ) denotes the standard basis of $\left.\mathbb{R}^{d}\right)$, Lemma A.2 shows because of $\left\|\varphi_{i} \cdot \gamma_{j}\right\|_{\mathcal{F} L^{1}}=$ $\left\|\varphi_{i}^{b} \cdot\left(\gamma_{j} \circ S_{i}\right)\right\|_{\mathcal{F} L^{1}}$ and $\frac{d+1}{\pi^{d}} \leq 1$ that

$$
\begin{aligned}
\left\|\varphi_{i} \cdot \gamma_{j}\right\|_{\mathcal{F} L^{1}} & \leq \max _{\alpha \in \mathbb{I}}\left\|\partial^{\alpha}\left(\varphi_{i}^{b} \cdot\left(\gamma_{j} \circ S_{i}\right)\right)\right\|_{L^{1}} \leq c_{0} \cdot\left|\operatorname{det} C_{j}\right|^{-1} . \\
& \left\|\left.\max _{|\nu| \leq d+1}\left|\partial^{v}\right| \mathcal{F} g_{i, j}^{\natural}\right|^{2} \mid\right\|_{L^{1}\left(Q_{i}^{\prime}\right)} \\
& \leq c_{0} c_{1} \cdot\left|\operatorname{det} C_{j}\right|^{-1}\left\|\left._{|\nu| \leq d+1}\left|\partial^{v}\right| \mathcal{F} g_{i, j}^{\natural}\right|^{2} \mid\right\|_{L^{d+1}\left(Q_{i}^{\prime}\right)},
\end{aligned}
$$

where $c_{1}=c_{1}(\mathcal{Q}, d)>0$ is a constant satisfying $\|\cdot\|_{L^{1}\left(Q_{i}^{\prime}\right)} \leq c_{1} \cdot\|\cdot\|_{L^{d+1}\left(Q_{i}^{\prime}\right)}$ for all $i \in I$, which exists since the $\left(Q_{i}^{\prime}\right)_{i \in I}$ are uniformly bounded. Estimate (6.6) implies that

$$
\sup _{i \in I} \sum_{j \in J}\left\|\varphi_{i} \cdot \gamma_{j}\right\|_{\text {sup }} \leq \sup _{i \in I} \sum_{j \in J}\left\|\varphi_{i} \cdot \gamma_{j}\right\|_{\mathcal{F} L^{1}} \leq c_{0} c_{1} \cdot M<\infty
$$

where $M$ is as in (6.3). This guarantees the locally uniform convergence on $\mathcal{O}$ of the series $t_{0}=\sum_{j \in J} \gamma_{j}$. Indeed, if $\xi \in \mathcal{O}$ is arbitrary, then $\xi \in Q_{i}$ for some $i \in I$ where $Q_{i}$ is open; furthermore, $\sum_{\ell \in i^{*}} \varphi_{\ell} \equiv 1$ on $Q_{i}$ and hence $\sum_{j \in J}\left\|\gamma_{j}\right\|_{L^{\infty}\left(Q_{i}\right)} \leq$ $\sum_{j \in J} \sum_{\ell \in i^{*}}\left\|\varphi_{\ell} \cdot \gamma_{j}\right\|_{\text {sup }}<\infty$, which shows that the series $t_{0}=\sum_{j \in J} \gamma_{j}$ converges uniformly on $Q_{i}$. By locally uniform convergence, we see that $t_{0}$ is continuous on $\mathcal{O}$. Eq. (4.1) shows that $A \leq t_{0} \leq B$ almost everywhere on $\mathcal{O}$; since $\mathcal{O}$ is open and $t_{0}$ continuous, this estimate necessarily holds pointwise on $\mathcal{O}$.

Finally, since $\operatorname{supp} \varphi_{i} \subset \mathcal{O}$ is compact, we see $\varphi_{i} t_{0}=\sum_{j \in J} \varphi_{i} \gamma_{j}$ with uniform convergence of the series, and hence with convergence in $L^{1}\left(\widehat{\mathbb{R}}^{d}\right)$, since all summands have support in the fixed compact set $\overline{Q_{i}} \subset \mathcal{O}$. Thus, $\mathcal{F}^{-1}\left(\varphi_{i} t_{0}\right)=\sum_{j \in J} \mathcal{F}^{-1}\left(\varphi_{i} \gamma_{j}\right)$, which leads to the estimate $\sup _{i \in I}\left\|\mathcal{F}^{-1}\left(\varphi_{i} t_{0}\right)\right\|_{L^{1}} \leq \sup _{i \in I} \sum_{j \in J}\left\|\varphi_{i} \cdot \gamma_{j}\right\|_{\mathcal{F} L^{1}} \leq$ $c_{0} c_{1} \cdot M<\infty$. Thus, $t_{0}$ is tame, so that Proposition 5.7 shows that $T_{0}=\Phi_{t_{0}}$ : $\mathcal{D}\left(\mathcal{Q}, L^{p}, \ell_{w}^{q}\right) \rightarrow \mathcal{D}\left(\mathcal{Q}, L^{p}, \ell_{w}^{q}\right)$ is well-defined and bounded.

Step 2. In this step, we prepare for applying Lemma A.4; we cannot apply it directly, since $t_{0}$ might not be $C^{d+1}$. Thus, we will construct a sequence $\left(g_{N}\right)_{N \in \mathbb{N}}$ of smooth functions approximating $t_{0}$. We will then apply Lemma A.4 to the $g_{N}$ in Step 3. 
For the construction of the $\left(g_{N}\right)_{N \in \mathbb{N}}$, first note that $J$ is infinite; indeed, we have $\widehat{g_{j}} \in C_{0}\left(\widehat{\mathbb{R}}^{d}\right)$ for all $j \in J$ since $g_{j} \in L^{1}\left(\mathbb{R}^{d}\right)$; thus, (4.1) can only hold if $J$ is infinite. Since $J$ is countable, we thus have $J=\left\{j_{n}: n \in \mathbb{N}\right\}$ for certain pairwise distinct $j_{n} \in J$. With this, define $g_{N}:=\sum_{n=1}^{N} \gamma_{j_{n}} \in C^{\infty}\left(\widehat{\mathbb{R}}^{d}\right)$. As seen in Step 1, $g_{N} \rightarrow t_{0}$ locally uniformly on $\mathcal{O}$. Since $0<A \leq t_{0} \leq B$ on $\mathcal{O}$, this easily implies $G_{N} \rightarrow \frac{1}{t_{0}}$ locally uniformly on $\mathcal{O}$, where we defined

$$
G_{N}: \mathcal{O} \rightarrow \mathbb{R}, \xi \mapsto \begin{cases}\left(g_{N}(\xi)\right)^{-1}, & \text { if } g_{N}(\xi) \neq 0 \\ 0, & \text { otherwise }\end{cases}
$$

Thus, $\varphi_{i} \cdot G_{N} \rightarrow \varphi_{i} \cdot t_{0}^{-1}$ in $L^{1}\left(\widehat{\mathbb{R}}^{d}\right)$, and hence $\mathcal{F}^{-1}\left(\varphi_{i} G_{N}\right) \rightarrow \mathcal{F}^{-1}\left(\varphi_{i} \cdot t_{0}^{-1}\right)$ uniformly as $N \rightarrow \infty$. Therefore, Fatou's lemma shows that

$$
\left\|\mathcal{F}^{-1}\left(\varphi_{i} \cdot t_{0}^{-1}\right)\right\|_{L^{1}} \leq \liminf _{N \rightarrow \infty}\left\|\mathcal{F}^{-1}\left(\varphi_{i} G_{N}\right)\right\|_{L^{1}}=\liminf _{N \rightarrow \infty}\left\|\varphi_{i}^{b} \cdot\left(G_{N} \circ S_{i}\right)\right\|_{\mathcal{F} L^{1}}
$$

Step 3. We next estimate $\lim \inf _{N \rightarrow \infty}\left\|\varphi_{i}^{b} \cdot\left(G_{N} \circ S_{i}\right)\right\|_{\mathcal{F} L^{1}}$. Define

$$
K_{i}^{(N)}: S_{i}^{-1}(\mathcal{O}) \rightarrow[0, \infty), \xi \mapsto \sum_{n=1}^{N} \max _{|\alpha| \leq d+1}\left|\partial^{\alpha}\left(\gamma_{j_{n}} \circ S_{i}\right)(\xi)\right|
$$

Let $V_{i} \subset \mathcal{O}$ be open and bounded with $\overline{Q_{i}} \subset V_{i} \subset \overline{V_{i}} \subset \mathcal{O}$ and let $\varepsilon \in(0,1)$. Since $g_{N} \rightarrow t_{0}$ uniformly on $V_{i}$ and $t_{0} \geq A>0$ on $\mathcal{O} \supset V_{i}$, there is $N_{0}=N_{0}(i, \varepsilon) \in \mathbb{N}$ such that $g_{N} \geq(1-\varepsilon) A=: A_{\varepsilon}$ on $V_{i}$ for all $N \geq N_{0}$. Note that $K_{i}^{(N)}(\xi) \geq$ $\sum_{n=1}^{N} \gamma_{j_{n}}\left(S_{i} \xi\right)=g_{N}\left(S_{i} \xi\right) \geq A_{\varepsilon}$ for $\xi \in S_{i}^{-1}\left(V_{i}\right)$ and $N \geq N_{0}$.

Define $U_{i}:=S_{i}^{-1}\left(V_{i}\right)$, fix $\xi^{(0)} \in U_{i}$ and $\ell \in \underline{d}$, set

$$
U:=\left\{\xi \in \widehat{\mathbb{R}}:\left(\xi_{1}^{(0)}, \ldots, \xi_{\ell-1}^{(0)}, \xi, \xi_{\ell+1}^{(0)}, \ldots, \xi_{d}^{(0)}\right) \in U_{i}\right\}
$$

and, for $N \geq N_{0}$, let $f_{N}: U \rightarrow\left[A_{\varepsilon}, \infty\right), \xi \mapsto\left(g_{N} \circ S_{i}\right)\left(\xi_{1}^{(0)}, \ldots, \xi_{\ell-1}^{(0)}, \xi, \xi_{\ell+1}^{(0)}, \ldots\right.$, $\left.\xi_{d}^{(0)}\right)$, noting that $\left|f_{N}^{(m)}\left(\xi_{\ell}^{(0)}\right)\right| \leq K_{i}^{(N)}\left(\xi^{(0)}\right)$ for all $m \in \underline{d+1}$. Hence, Lemma A.4 shows for all $m \in \underline{d+1}$ that

$$
\begin{aligned}
\left|\frac{\partial^{m}}{\partial \xi_{\ell}^{m}}\right|_{\xi=\xi^{(0)}}\left(G_{N} \circ S_{i}\right)(\xi) \mid & =\left|\frac{d^{m}}{d \xi^{m}}\right|_{\xi=\xi_{\ell}^{(0)}} \frac{1}{f_{N}(\xi)} \mid \\
\leq & C_{d+1} \cdot A_{\varepsilon}^{-1} \cdot \max \left\{A_{\varepsilon}^{-1} \cdot K_{i}^{(N)}\left(\xi^{(0)}\right),\right. \\
& \left.\left(A_{\varepsilon}^{-1} \cdot K_{i}^{(N)}\left(\xi^{(0)}\right)\right)^{m}\right\} \\
\leq & C_{d+1} \cdot A_{\varepsilon}^{-(d+2)} \cdot\left(K_{i}^{(N)}\left(\xi^{(0)}\right)\right)^{d+1},
\end{aligned}
$$

where $C_{d+1}$ is as in Lemma A.4. 
Since $\xi^{(0)} \in U_{i}$ was arbitrary, we have thus shown, for all $\xi \in U_{i}$ and $N \geq N_{0}$,

$$
\max _{\ell \in \underline{d}} \max _{0 \leq m \leq d+1}\left|\partial_{\ell}^{m}\left(G_{N} \circ S_{i}\right)(\xi)\right| \leq C_{d+1} \cdot A_{\varepsilon}^{(-d+2)} \cdot\left(K_{i}^{(N)}(\xi)\right)^{d+1}
$$

Finally, since $\varphi_{i}^{b}=\varphi_{i} \circ S_{i}$ vanishes outside of $Q_{i}^{\prime}=S_{i}^{-1}\left(Q_{i}\right) \subset S_{i}^{-1}\left(V_{i}\right)=U_{i}$, the Leibniz rule shows

$$
\begin{aligned}
\left|\partial_{\ell}^{m}\left(\varphi_{i}^{b} \cdot\left(G_{N} \circ S_{i}\right)\right)(\xi)\right| & \leq \sum_{s=0}^{m}\left(\begin{array}{c}
m \\
s
\end{array}\right)\left|\partial_{\ell}^{m-s} \varphi_{i}^{b}(\xi)\right|\left|\partial_{\ell}^{s}\left(G_{N} \circ S_{i}\right)(\xi)\right| \\
& \leq c_{0} C_{d+1} \cdot A_{\varepsilon}^{-(d+2)} \cdot\left(K_{i}^{(N)}(\xi)\right)^{d+1} \cdot \mathbb{1}_{Q_{i}^{\prime}}(\xi)
\end{aligned}
$$

for all $\xi \in \widehat{\mathbb{R}}^{d}, \ell \in \underline{d}, 0 \leq m \leq d+1$, and $N \geq N_{0}$. Thus, Lemma A.2 shows

$$
\begin{aligned}
\| \varphi_{i}^{\mathrm{b}} \cdot & \left(G_{N} \circ S_{i}\right) \|_{\mathcal{F} L^{1}} \\
& \leq \frac{d+1}{\pi^{d}} \max _{\substack{\ell \in d \\
0 \leq m \leq d+1}}\left\|\partial_{\ell}^{m}\left(\varphi_{i}^{\mathrm{b}} \cdot\left(G_{N} \circ S_{i}\right)\right)\right\|_{L^{1}} \\
& \leq \frac{d+1}{\pi^{d}} \cdot c_{0} C_{d+1} \cdot A_{\varepsilon}^{-(d+2)}\left\|K_{i}^{(N)}\right\|_{L^{d+1}\left(Q_{i}^{\prime}\right)}^{d+1} \\
& \leq \frac{d+1}{\pi^{d}} \cdot c_{0} C_{d+1} \cdot A_{\varepsilon}^{-(d+2)} \cdot\left(\sum_{j \in J}\left|\operatorname{det} C_{j}\right|^{-1}\left\|\left._{|\alpha| \leq d+1} \max \left|\partial^{\alpha}\right| \mathcal{F} g_{i, j}^{\natural}\right|^{2} \mid\right\|_{L^{d+1}\left(Q_{i}^{\prime}\right)}\right)^{d+1} \\
& \leq \frac{d+1}{\pi^{d}} \cdot c_{0} C_{d+1} \cdot A_{\varepsilon}^{-(d+2)} \cdot M^{d+1} .
\end{aligned}
$$

Since this holds for all $N \geq N_{0}=N_{0}(i, \varepsilon)$, and since $A_{\varepsilon}=(1-\varepsilon) A$ where $\varepsilon \in(0,1)$ is arbitrary, we thus see by virtue of Eq. (6.7) that

$$
\left\|\mathcal{F}^{-1}\left(\varphi_{i} \cdot t_{0}^{-1}\right)\right\|_{L^{1}} \leq \frac{d+1}{\pi^{d}} \cdot c_{0} C_{d+1} \cdot A^{-(d+2)} \cdot M^{d+1}<\infty
$$

for all $i \in I$. Hence, $t_{0}^{-1}$ is tame, and Proposition 5.7 shows that $\Phi_{t_{0}^{-1}}$ : $\mathcal{D}\left(\mathcal{Q}, L^{p}, \ell_{w}^{q}\right) \rightarrow \mathcal{D}\left(\mathcal{Q}, L^{p}, \ell_{w}^{q}\right)$ is well-defined and bounded, with operator norm bounded by the right-hand side of Eq. (6.4).

Step 4. Proposition 5.7(iv) shows $\Phi_{t_{0}^{-1}} \Phi_{t_{0}}=\Phi_{1}=\Phi_{t_{0}} \Phi_{t_{0}^{-1}}$, where $1: \mathcal{O} \rightarrow$ $\mathbb{R}, \xi \mapsto 1$. Directly from the definition of $\Phi_{1}$ in Proposition 5.7, we see $\Phi_{1} f=f$ for all $f \in \mathcal{D}\left(\mathcal{Q}, L^{p}, \ell_{w}^{q}\right)$. Hence, $T_{0}: \mathcal{D}\left(\mathcal{Q}, L^{p}, \ell_{w}^{q}\right) \rightarrow \mathcal{D}\left(\mathcal{Q}, L^{p}, \ell_{w}^{q}\right)$ is boundedly invertible with $T_{0}^{-1}=\Phi_{t_{0}^{-1}}$.

\subsection{The Remainder Term}

The next (technical) result provides an estimate of the operator norm of the remainder term $R_{0}: \mathcal{D}\left(\mathcal{Q}, L^{p}, \ell_{w}^{q}\right) \rightarrow \mathcal{D}\left(\mathcal{Q}, L^{p}, \ell_{w}^{q}\right)$ considered in Corollary 5.9. Here, we 


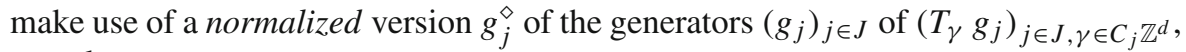
namely

$$
g_{j}^{\diamond}:=\left|\operatorname{det} B_{j}\right|^{-1 / 2} \cdot\left(M_{-c_{j}} g_{j}\right) \circ B_{j}^{-t}
$$

for invertible affine-linear maps $U_{j}=B_{j}(\cdot)+c_{j}$; note that $\widehat{g_{j}^{\diamond}}=\left|\operatorname{det} B_{j}\right|^{1 / 2} \cdot \widehat{g_{j}} \circ U_{j}$. Lemma 6.4 Let $\mathcal{Q}=\left(S_{i}\left(Q_{i}^{\prime}\right)\right)_{i \in I}=\left(A_{i}\left(Q_{i}^{\prime}\right)+b_{i}\right)_{i \in I}$ be an affinely generated cover of an open set $\mathcal{O} \subset \widehat{\mathbb{R}}^{d}$ of full measure. Let $\Phi=\left(\varphi_{i}\right)_{i \in I}$ be a regular partition of unity subordinate to $\mathcal{Q}$, and let $w=\left(w_{i}\right)_{i \in I}$ be a $\mathcal{Q}$-moderate weight. Let $\left(T_{\gamma} g_{j}\right)_{j \in J, \gamma \in C_{j} \mathbb{Z}^{d}}$ be a generalized shift-invariant system. Furthermore, assume that $\left(T_{\gamma} g_{j}\right)_{j \in J, \gamma \in C_{j} \mathbb{Z}^{d}}$ is $(w, v, \Phi)$-adapted for some weight $v=\left(v_{j}\right)_{j \in J}$, and assume that the function $t_{0}$ introduced in Eq. (5.2) is tame.

Suppose that there is a family $\left(U_{j}\right)_{j \in J}$ of invertible affine-linear maps $U_{j}=B_{j}(\cdot)+$ $c_{j}$ and a weight $v=\left(v_{j}\right)_{j \in J}$ such that the Fourier transform of $g_{j}^{\diamond}=\left|\operatorname{det} B_{j}\right|^{-1 / 2}$. $\left(M_{-c_{j}} g_{j}\right) \circ B_{j}^{-t}$ can be factorized as $\mathcal{F} g_{j}^{\diamond}=h_{j, 1} \cdot h_{j, 2}$ with $h_{j, 1}, h_{j, 2} \in C^{d+1}\left(\widehat{\mathbb{R}}^{d}\right)$ satisfying

$$
\max _{|\alpha| \leq d+1}\left|\partial^{\alpha} h_{j, 2}(\xi)\right| \leq C^{\prime} \cdot(1+|\xi|)^{-(d+1)} \text { for } \xi \in \widehat{\mathbb{R}}^{d}
$$

Moreover, suppose that $Y=\left(a_{i, j} X_{i, j}\right)_{i \in I, j \in J}$ and $Z=\left(b_{i, j} X_{i, j}\right)_{i \in I, j \in J}$ are of Schur-type, where

$$
\begin{aligned}
a_{i, j}= & \max \left\{1, \frac{w_{i}}{v_{j}}\right\}\left|\operatorname{det} B_{j}^{t} C_{j}\right|^{-1} \max \left\{1,\left|A_{i}^{-1}\left(b_{i}-c_{j}\right)\right|^{d+1}\right\} \\
& \max \left\{1,\left\|A_{i}^{-1} B_{j}\right\|^{d+1}\right\}\left\|C_{j}^{t} A_{i}\right\|^{d+1}
\end{aligned}
$$

and

$$
\begin{gathered}
b_{i, j}=\max \left\{1, \frac{v_{j}}{w_{i}}\right\} \max \left\{1,\left|A_{i}^{-1}\left(b_{i}-c_{j}\right)\right|\right\} \max \left\{1,\left\|A_{i}^{-1} B_{j}\right\|^{d+1}\right\} \\
\max \left\{\left\|C_{j}^{t} A_{i}\right\|,\left\|C_{j}^{t} A_{i}\right\|^{d+1}\right\},
\end{gathered}
$$

and

$$
X_{i, j}:=\max \left\{1,\left\|B_{j}^{-1} A_{i}\right\|^{d+1}\right\} \int_{Q_{i}^{\prime}|\alpha| \leq d+1} \max _{\mid}\left|\left(\partial^{\alpha} h_{j, 1}\right)\left(U_{j}^{-1} S_{i}(\xi)\right)\right| d \xi .
$$

Then, for all $p, q \in[1, \infty]$, the operator $R_{0}: \mathcal{D}\left(\mathcal{Q}, L^{p}, \ell_{w}^{q}\right) \rightarrow \mathcal{D}\left(\mathcal{Q}, L^{p}, \ell_{w}^{q}\right)$ of Corollary 5.9 is bounded, with $\left\|R_{0}\right\|_{\mathrm{op}} \leq C_{0} C_{p, q}\left\|\Gamma_{\mathcal{Q}}\right\|_{\ell_{w}^{q} \rightarrow \ell_{w}^{q}} \cdot\left(C^{\prime}\right)^{2} \cdot\|Y\|_{\text {Schur }}\|Z\|_{\text {Schur }}$, where

$$
C_{0}:=24 \pi^{2}\left(\frac{8 d}{\pi}\right)^{2 d+2} 12^{d}(d+1)^{3} \max \left\{1, R_{\mathcal{Q}}^{d+2}\right\} \max _{|\alpha| \leq d+1} C_{\mathcal{Q}, \Phi, \alpha}^{2}
$$

with $R_{\mathcal{Q}}:=\max _{i \in I} \sup _{\xi \in Q_{i}^{\prime}}|\xi|$ and $C_{p, q}:=1$ if $\max \{p, q\}<\infty$ and $C_{p, q}:=$ $C_{\Phi} \cdot\left\|\Gamma_{\mathcal{Q}}\right\|_{\ell_{w}^{q} \rightarrow \ell_{w}^{q}}^{2}$ otherwise. 
Proof For brevity, $\operatorname{set} v(x):=\max \{1, x\}$ for $x \in[0, \infty)$, and note $v(x y) \leq v(x) v(y)$. This implies $v\left(w_{i} / w_{\ell}\right) \leq v\left(w_{i} / v_{j}\right) \cdot v\left(v_{j} / w_{\ell}\right)$, an estimate that we will employ frequently.

According to Proposition 5.8 and Corollary 5.10, it suffices to estimate

$$
\begin{aligned}
L_{1} & =\sup _{i \in I} \sum_{\ell \in I} \sum_{j \in J} \sum_{k \in \mathbb{Z}^{d} \backslash\{0\}} v\left(\frac{w_{i}}{w_{\ell}}\right) K_{i, \ell, j, k} \text { and } L_{2} \\
& =\sup _{\ell \in I} \sum_{i \in I} \sum_{j \in J} \sum_{k \in \mathbb{Z}^{d} \backslash\{0\}} v\left(\frac{w_{i}}{w_{\ell}}\right) K_{i, \ell, j, k}
\end{aligned}
$$

where $K_{i, \ell, j, k}:=\left|\operatorname{det} C_{j}\right|^{-1} \cdot\left\|\widehat{g_{j}} \cdot \widehat{g_{j}}\left(\cdot-C_{j}^{-t} k\right) \cdot \varphi_{i}\left(\cdot-C_{j}^{-t} k\right) \cdot \varphi_{\ell}\right\|_{\mathcal{F} L^{1}}$. In order to do so, note that $\widehat{g_{j}}=\left|\operatorname{det} B_{j}\right|^{-1 / 2} \cdot\left(\mathcal{F} g_{j}^{\diamond}\right) \circ U_{j}^{-1}$. Hence, since $\mathcal{F} g_{j}^{\diamond}=h_{j, 1} \cdot h_{j, 2}$ by assumption, the term $K_{i, \ell, j, k}$ can be estimated as follows:

$$
\begin{aligned}
& K_{i, \ell, j, k}=\left|\operatorname{det} B_{j}^{t} C_{j}\right|^{-1}\left\|\left(\overline{\mathcal{F} g_{j}^{\diamond}} \circ U_{j}^{-1}\right) \cdot T_{C_{j}^{-t} k}\left(\left(\mathcal{F} g_{j}^{\diamond}\right) \circ U_{j}^{-1}\right) \cdot\left(T_{C_{j}^{-t} k} \varphi_{i}\right) \cdot \varphi_{\ell}\right\|_{\mathcal{F} L^{1}} \\
& \leq\left|\operatorname{det} B_{j}^{t} C_{j}\right|^{-1}\left\|T_{C_{j}^{-t} k}\left(\varphi_{i} \cdot\left(h_{j, 1} \circ U_{j}^{-1}\right)\right) \cdot\left(\overline{h_{j, 2}} \circ U_{j}^{-1}\right)\right\|_{\mathcal{F} L^{1}} \\
& \cdot\left\|\varphi_{\ell} \cdot\left(\overline{h_{j, 1}} \circ U_{j}^{-1}\right) \cdot T_{C_{j}^{-t} k}\left(h_{j, 2} \circ U_{j}^{-1}\right)\right\|_{\mathcal{F} L^{1}} \\
& =:\left|\operatorname{det} B_{j}^{t} C_{j}\right|^{-1} \cdot K_{i, j, k}^{(1)} \cdot K_{\ell, j, k}^{(2)} .
\end{aligned}
$$

Using the preceding estimate, one can bound $L_{1}$ from Eq. (6.10) as follows:

$$
\begin{aligned}
L_{1} & =\sup _{i \in I} \sum_{j \in J, \ell \in I} \sum_{k \in \mathbb{Z}^{d} \backslash\{0\}} v\left(\frac{w_{i}}{w_{\ell}}\right) K_{i, \ell, j, k} \\
& \leq \sup _{i \in I} \sum_{j \in J}\left[\left(\sum_{\ell \in I} \sum_{k \in \mathbb{Z}^{d} \backslash\{0\}} v\left(\frac{v_{j}}{w_{\ell}}\right) K_{\ell, j, k}^{(2)}\right) \cdot\left|\operatorname{det} B_{j}^{t} C_{j}\right|^{-1} v\left(\frac{w_{i}}{v_{j}}\right) \sup _{k \in \mathbb{Z}^{d} \backslash\{0\}} K_{i, j, k}^{(1)}\right] \\
& \leq\left(\sup _{j \in J} \sum_{\ell \in I} v\left(\frac{v_{j}}{w_{\ell}}\right) \sum_{k \in \mathbb{Z}^{d} \backslash\{0\}} K_{\ell, j, k}^{(2)}\right) \cdot \sup _{i \in I} \sum_{j \in J}\left(v\left(\frac{w_{i}}{v_{j}}\right)\left|\operatorname{det} B_{j}^{t} C_{j}\right|^{-1} \sup _{k \in \mathbb{Z}^{d} \backslash\{0\}} K_{i, j, k}^{(1)}\right) .
\end{aligned}
$$

A similar calculation gives

$$
\begin{aligned}
L_{2} & \leq\left(\sup _{\ell \in I} \sum_{j \in J} v\left(\frac{v_{j}}{w_{\ell}}\right) \sum_{k \in \mathbb{Z}^{d} \backslash\{0\}} K_{\ell, j, k}^{(2)}\right) . \\
& \sup _{j \in J} \sum_{i \in I}\left(v\left(\frac{w_{i}}{v_{j}}\right)\left|\operatorname{det} B_{j}^{t} C_{j}\right|^{-1} \sup _{k \in \mathbb{Z}^{d} \backslash\{0\}} K_{i, j, k}^{(1)}\right) .
\end{aligned}
$$

The remainder of the proof is divided into four steps: 
Step 1. Estimates for $K_{i, j, k}^{(1)}$ and $K_{\ell, j, k}^{(2)}$. For $j \in J$ and $k \in \mathbb{Z}^{d}$, set $H_{j, k}:=\overline{h_{j, 1}}$. $T_{B_{j}^{-1} C_{j}^{-t} k} h_{j, 2}$. Since $T_{\xi}\left(g \circ U_{j}^{-1}\right)=\left(T_{B_{j}^{-1} \xi} g\right) \circ U_{j}^{-1}$ for any $\xi \in \widehat{\mathbb{R}}^{d}$ and $g: \widehat{\mathbb{R}}^{d} \rightarrow \mathbb{C}$, it follows that

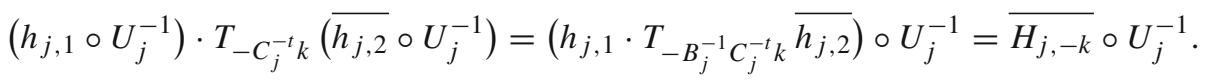

Using the normalization $\varphi_{i}^{b}=\varphi_{i} \circ S_{i}$ of $\varphi_{i}$, a direct calculation shows

$$
\begin{array}{r}
K_{i, j, k}^{(1)}=\left\|\varphi_{i} \cdot\left(h_{j, 1} \circ U_{j}^{-1}\right) \cdot T_{-C_{j}^{-t} k}\left(\overline{h_{j, 2}} \circ U_{j}^{-1}\right)\right\|_{\mathcal{F} L^{1}} \\
=\left\|\varphi_{i}^{b} \cdot\left(\overline{H_{j,-k}} \circ U_{j}^{-1} \circ S_{i}\right)\right\|_{\mathcal{F} L^{1}} .
\end{array}
$$

Now, define $\zeta_{j}: \widehat{\mathbb{R}}^{d} \rightarrow[0, \infty), \xi \mapsto \max _{|\alpha| \leq d+1}\left|\partial^{\alpha} h_{j, 1}(\xi)\right|$. By applying Leibniz' rule, combined with the assumption $\max _{|\alpha| \leq d+1}\left|\partial^{\alpha} h_{j, 2}(\xi)\right| \leq C^{\prime} \cdot(1+|\xi|)^{-(d+1)}$ and the identity $\sum_{\beta \leq \alpha}\left(\begin{array}{l}\alpha \\ \beta\end{array}\right)=2^{|\alpha|}$, we see

$$
\left|\partial^{\alpha} H_{j, k}(\xi)\right| \leq 2^{|\alpha|} \cdot C^{\prime} \cdot\left(1+\left|\xi-B_{j}^{-1} C_{j}^{-t} k\right|\right)^{-(d+1)} \cdot \zeta_{j}(\xi)
$$

for all $\alpha \in \mathbb{N}_{0}^{d}$ with $|\alpha| \leq d+1$ and all $\xi \in \widehat{\mathbb{R}}^{d}$. This, together with Lemma A.3, yields that, for all $n \in \underline{d}$ and $m \in\{0, \ldots, d+1\}$,

$$
\begin{aligned}
& \left|\left[\partial_{n}^{m}\left(\overline{H_{j, k}} \circ U_{j}^{-1} \circ S_{i}\right)\right](\xi)\right| \\
& \leq\left\|B_{j}^{-1} A_{i}\right\|^{m} \cdot d^{m} \cdot \max _{\beta \in \mathbb{N}_{0}^{d} \text { with }|\beta|=m}\left|\left(\partial^{\beta} H_{j, k}\right)\left(U_{j}^{-1}\left(S_{i}(\xi)\right)\right)\right| \\
& \leq(2 d)^{d+1} C^{\prime} \max \left\{1,\left\|B_{j}^{-1} A_{i}\right\|^{d+1}\right\} \zeta_{j}\left(U_{j}^{-1}\left(S_{i}(\xi)\right)\right)\left(1+\mid U_{j}^{-1}\left(S_{i}(\xi)\right)\right. \\
& \left.-B_{j}^{-1} C_{j}^{-t} k \mid\right)^{-(d+1)} .
\end{aligned}
$$

Since $\Phi$ is a regular partition of unity, we have $\left|\partial^{\alpha} \varphi_{i}^{b}(\xi)\right| \leq C_{\mathcal{Q}, \Phi, \alpha} \cdot \mathbb{1}_{Q_{i}^{\prime}}(\xi)$ for all $\xi \in \widehat{\mathbb{R}}^{d}$ and $\alpha \in \mathbb{N}_{0}^{d}$. Thus, setting $C_{1}:=(4 d)^{d+1} C^{\prime} \cdot \max _{|\alpha| \leq d+1} C_{\mathcal{Q}, \Phi, \alpha}$ and invoking Leibniz's rule once more, we see that

$$
\begin{aligned}
& \left|\left[\partial_{n}^{d+1}\left(\varphi_{i}^{\mathrm{b}} \cdot\left(\overline{H_{j,-k}} \circ U_{j}^{-1} \circ S_{i}\right)\right)\right](\xi)\right| \\
& \leq \sum_{m=0}^{d+1}\left(\begin{array}{c}
d+1 \\
m
\end{array}\right)\left|\partial_{n}^{d+1-m} \varphi_{i}^{\mathrm{b}}(\xi)\right| \cdot\left|\partial_{n}^{m}\left(\overline{H_{j,-k}} \circ U_{j}^{-1} \circ S_{i}\right)(\xi)\right| \\
& \leq C_{1} \max \left\{1,\left\|B_{j}^{-1} A_{i}\right\|^{d+1}\right\} \mathbb{1}_{Q_{i}^{\prime}}(\xi) \zeta_{j}\left(U_{j}^{-1}\left(S_{i}(\xi)\right)\right)\left(1+\mid U_{j}^{-1}\left(S_{i}(\xi)\right)\right. \\
& \left.\quad+B_{j}^{-1} C_{j}^{-t} k \mid\right)^{-(d+1)} .
\end{aligned}
$$


Clearly, the same overall estimate also holds for $\left|\left[\varphi_{i}^{b} \cdot\left(\overline{H_{j,-k}} \circ U_{j}^{-1} \circ S_{i}\right)\right](\xi)\right|$ itself instead of its derivative $\left|\partial_{n}^{d+1}\left(\varphi_{i}^{\mathrm{b}} \cdot\left(\overline{H_{j,-k}} \circ U_{j}^{-1} \circ S_{i}\right)\right)(\xi)\right|$. Thus, setting

$$
C_{2}:=(4 d / \pi)^{d+1} \cdot(d+1) \pi \cdot C^{\prime} \cdot \max _{|\alpha| \leq d+1} C_{\mathcal{Q}, \Phi, \alpha},
$$

we can apply Lemma A.2 and Eq. (6.13) to conclude

$$
\begin{aligned}
K_{i, j, k}^{(1)}= & \left\|\varphi_{i}^{b} \cdot\left(\overline{H_{j,-k}} \circ U_{j}^{-1} \circ S_{i}\right)\right\|_{\mathcal{F} L^{1}} \leq \frac{d+1}{\pi^{d}} \cdot \max _{\alpha \in \mathbb{I}}\left\|\partial^{\alpha}\left(\varphi_{i}^{b} \cdot\left(\overline{H_{j,-k}} \circ U_{j}^{-1} \circ S_{i}\right)\right)\right\|_{L^{1}} \\
\leq & C_{2} \cdot \max \left\{1,\left\|B_{j}^{-1} A_{i}\right\|^{d+1}\right\} \cdot \int_{Q_{i}^{\prime}} \zeta_{j}\left(U_{j}^{-1}\left(S_{i}(\xi)\right)\right) \\
& \cdot\left(1+\left|U_{j}^{-1}\left(S_{i}(\xi)\right)+B_{j}^{-1} C_{j}^{-t} k\right|\right)^{-(d+1)} d \xi
\end{aligned}
$$

where $\mathbb{I}:=\{0\} \cup\left\{(d+1) \cdot e_{n}: n \in \underline{d}\right\}$. By similar arguments as for $K_{i, j, k}^{(1)}$, one obtains

$$
\begin{aligned}
K_{\ell, j, k}^{(2)} \leq C_{2} \cdot \max \{ & \left.1,\left\|B_{j}^{-1} A_{\ell}\right\|^{d+1}\right\} \\
& \cdot \int_{Q_{\ell}^{\prime}} \zeta_{j}\left(U_{j}^{-1}\left(S_{\ell}(\xi)\right)\right) \cdot\left(1+\left|U_{j}^{-1}\left(S_{\ell}(\xi)\right)-B_{j}^{-1} C_{j}^{-t} k\right|\right)^{-(d+1)} d \xi .
\end{aligned}
$$

Step 2. Estimating the supremum over $k \in \mathbb{Z}^{d} \backslash\{0\}$. Note that $|\xi| \leq\left\|A^{-1}\right\| \cdot|A \xi|$, and thus $|A \xi| \geq\left\|A^{-1}\right\|^{-1} \cdot|\xi|$ for any $\xi \in \widehat{\mathbb{R}}^{d}$ and $A \in \operatorname{GL}\left(\mathbb{R}^{d}\right)$. Hence,

$$
\begin{aligned}
\left|U_{j}^{-1}\left(S_{i}(\xi)\right) \pm B_{j}^{-1} C_{j}^{-t} k\right| & =\left|B_{j}^{-1}\left(S_{i}(\xi)-c_{j}\right) \pm B_{j}^{-1} C_{j}^{-t} k\right| \\
& =\left|B_{j}^{-1} A_{i}\left(\xi+A_{i}^{-1}\left(b_{i}-c_{j}\right) \pm A_{i}^{-1} C_{j}^{-t} k\right)\right| \\
& \geq\left\|A_{i}^{-1} B_{j}\right\|^{-1} \cdot\left|\xi+A_{i}^{-1}\left(b_{i}-c_{j}\right) \pm A_{i}^{-1} C_{j}^{-t} k\right|
\end{aligned}
$$

This implies for arbitrary $i \in I, \xi \in Q_{i}^{\prime}, k \in \mathbb{Z}^{d} \backslash\{0\}$, and $j \in J$ that

$$
\begin{aligned}
& \left\|C_{j}^{t} A_{i}\right\|^{-1} \leq 1+\left|A_{i}^{-1} C_{j}^{-t} k\right| \leq 1+\left|\xi+A_{i}^{-1}\left(b_{i}-c_{j}\right) \pm A_{i}^{-1} C_{j}^{-t} k\right|+|\xi| \\
& +\left|A_{i}^{-1}\left(b_{i}-c_{j}\right)\right| \\
\leq & 3 \max \left\{1, R_{\mathcal{Q}}\right\} \max \left\{1,\left|A_{i}^{-1}\left(b_{i}-c_{j}\right)\right|\right\}\left(1+\left|\xi+A_{i}^{-1}\left(b_{i}-c_{j}\right) \pm A_{i}^{-1} C_{j}^{-t} k\right|\right) \\
\leq & 3 \max \left\{1, R_{\mathcal{Q}}\right\} \max \left\{1,\left|A_{i}^{-1}\left(b_{i}-c_{j}\right)\right|\right\}\left(1+\left\|A_{i}^{-1} B_{j}\right\| \cdot\left|U_{j}^{-1}\left(S_{i}(\xi)\right) \pm B_{j}^{-1} C_{j}^{-t} k\right|\right) \\
\leq & 3 \max \left\{1, R_{\mathcal{Q}}\right\} \max \left\{1,\left|A_{i}^{-1}\left(b_{i}-c_{j}\right)\right|\right\} \\
& \quad \max \left\{1,\left\|A_{i}^{-1} B_{j}\right\|\right\}\left(1+\left|U_{j}^{-1}\left(S_{i}(\xi)\right) \pm B_{j}^{-1} C_{j}^{-t} k\right|\right) .
\end{aligned}
$$


Setting $C_{3}:=3^{d+1} \cdot \max \left\{1, R_{\mathcal{Q}}^{d+1}\right\}$, the preceding estimate implies

$$
\begin{aligned}
& \sup _{k \in \mathbb{Z}^{d} \backslash\{0\}}\left(1+\left|U_{j}^{-1}\left(S_{i}(\xi)\right) \pm B_{j}^{-1} C_{j}^{-t} k\right|\right)^{-(d+1)} \\
& \quad \leq C_{3} \max \left\{1,\left|A_{i}^{-1}\left(b_{i}-c_{j}\right)\right|^{d+1}\right\} \max \left\{1,\left\|A_{i}^{-1} B_{j}\right\|^{d+1}\right\}\left\|C_{j}^{t} A_{i}\right\|^{d+1}
\end{aligned}
$$

for all $i \in I, \xi \in Q_{i}^{\prime}$, and $j \in J$. Using this, and the estimates for $K_{i, j, k}^{(n)}$ that we derived in Step 1, we see that

$$
\begin{aligned}
\sup _{k \in \mathbb{Z}^{d} \backslash\{0\}} K_{i, j, k}^{(n)} & \leq C_{2} C_{3} \max \left\{1,\left|A_{i}^{-1}\left(b_{i}-c_{j}\right)\right|^{d+1}\right\} \max \{1, \\
& \left.\left\|A_{i}^{-1} B_{j}\right\|^{d+1}\right\}\left\|C_{j}^{t} A_{i}\right\|^{d+1} X_{i, j} \\
& =C_{2} C_{3}\left|\operatorname{det} B_{j}^{t} C_{j}\right|\left(v\left(w_{i} / v_{j}\right)\right)^{-1} \cdot Z_{i, j}
\end{aligned}
$$

for $n \in\{1,2\}, i \in I$, and $j \in J$.

Step 3. Estimating the sum over $k \in \mathbb{Z}^{d} \backslash\{0\}$. Estimate (6.15) implies

$$
\begin{aligned}
1+\left|U_{j}^{-1}\left(S_{i}(\xi)\right)+B_{j}^{-1} C_{j}^{-t} k\right| \geq & 1+\left\|A_{i}^{-1} B_{j}\right\|^{-1} \cdot\left|\xi+A_{i}^{-1}\left(b_{i}-c_{j}\right)+A_{i}^{-1} C_{j}^{-t} k\right| \\
\geq\left(\max \left\{1,\left\|A_{i}^{-1} B_{j}\right\|\right\}\right)^{-1} & \cdot\left(1+\mid \xi+A_{i}^{-1}\left(b_{i}-c_{j}\right)+A_{i}^{-1} C_{j}^{-t} k\right) .
\end{aligned}
$$

By combining this estimate with Corollary D.2, we see for any $\xi \in Q_{i}^{\prime}$ that

$$
\begin{aligned}
& \sum_{k \in \mathbb{Z}^{d} \backslash\{0\}}\left(1+\left|U_{j}^{-1}\left(S_{i}(\xi)\right)+B_{j}^{-1} C_{j}^{-t} k\right|\right)^{-(d+1)} \\
& \leq \max \left\{1,\left\|A_{i}^{-1} B_{j}\right\|^{d+1}\right\} \sum_{k \in \mathbb{Z}^{d} \backslash\{0\}}\left(1+\left|\xi+A_{i}^{-1}\left(b_{i}-c_{j}\right)+A_{i}^{-1} C_{j}^{-t} k\right|\right)^{-(d+1)} \\
& \leq(d+1) 2^{3+4 d} \cdot \max \left\{1,\left\|A_{i}^{-1} B_{j}\right\|^{d+1}\right\} \\
& \quad \cdot\left(1+\left|\xi+A_{i}^{-1}\left(b_{i}-c_{j}\right)\right|\right) \cdot \max \left\{\left\|C_{j}^{t} A_{i}\right\|,\left\|C_{j}^{t} A_{i}\right\|^{d+1}\right\} \\
& \leq(d+1) 2^{3+4 d}\left(2+R_{\mathcal{Q}}\right) \cdot \max \left\{1,\left\|A_{i}^{-1} B_{j}\right\|^{d+1}\right\} \\
& \cdot \max \left\{1,\left|A_{i}^{-1}\left(b_{i}-c_{j}\right)\right|\right\} \cdot \max \left\{\left\|C_{j}^{t} A_{i}\right\|,\left\|C_{j}^{t} A_{i}\right\|^{d+1}\right\} .
\end{aligned}
$$

Here, we used in the last step that $|\xi| \leq R_{\mathcal{Q}}$ since $\xi \in Q_{i}^{\prime}$. 
By combining this estimate with the estimate for $K_{i, j, k}^{(n)}$ from Step 1, we see for $n \in\{1,2\}$ and arbitrary $i \in I$ and $j \in J$ that

$$
\begin{aligned}
& \sum_{k \in \mathbb{Z}^{d} \backslash\{0\}} K_{i, j, k}^{(n)} \\
\leq & C_{2} \max \left\{1,\left\|B_{j}^{-1} A_{i}\right\|^{d+1}\right\} \\
& \quad \int_{Q_{i}^{\prime}} \zeta_{j}\left(U_{j}^{-1}\left(S_{i}(\xi)\right)\right) \sum_{k \in \mathbb{Z}^{d} \backslash\{0\}}\left(1+\left|U_{j}^{-1}\left(S_{i}(\xi)\right)+B_{j}^{-1} C_{j}^{-t} k\right|\right)^{-(d+1)} d \xi \\
\leq & C_{4} \max \left\{1,\left\|A_{i}^{-1} B_{j}\right\|^{d+1}\right\} \max \left\{1,\left|A_{i}^{-1}\left(b_{i}-c_{j}\right)\right|\right\} \cdot \max \left\{\left\|C_{j}^{t} A_{i}\right\|,\left\|C_{j}^{t} A_{i}\right\|^{d+1}\right\} X_{i, j} \\
= & C_{4} \cdot\left(v\left(v_{j} / w_{i}\right)\right)^{-1} \cdot Y_{i, j},
\end{aligned}
$$

where we defined $C_{4}:=(d+1) \cdot 2^{3+4 d} \cdot\left(2+R_{\mathcal{Q}}\right) \cdot C_{2}$.

Step 4. Completing the proof. Combining the two estimates (6.11) and (6.12) with the estimates obtained in Equations (6.17) and (6.16), we conclude that

$$
\begin{aligned}
L_{1} & \leq\left(\sup _{j \in J} \sum_{\ell \in I} v\left(\frac{v_{j}}{w_{\ell}}\right) \sum_{k \in \mathbb{Z}^{d} \backslash\{0\}} K_{\ell, j, k}^{(2)}\right) \cdot \sup _{i \in I} \sum_{j \in J}\left(v\left(\frac{w_{i}}{v_{j}}\right)\left|\operatorname{det} B_{j}^{t} C_{j}\right|^{-1} \sup _{k \in \mathbb{Z}^{d} \backslash\{0\}} K_{i, j, k}^{(1)}\right) \\
& \leq C_{2} C_{3} C_{4}\|Y\|_{\text {Schur }}\|Z\|_{\text {Schur }} \leq C_{0} \cdot\left(C^{\prime}\right)^{2} \cdot\|Y\|_{\text {Schur }}\|Z\|_{\text {Schur }} .
\end{aligned}
$$

The estimate $L_{2} \leq C_{0} \cdot\left(C^{\prime}\right)^{2} \cdot\|Y\|_{\text {Schur }}\|Z\|_{\text {Schur is obtained similarly. Hence, an }}$ application of Corollaries 5.9 and 5.10 gives $\left\|R_{0}\right\|_{\mathrm{op}} \leq C_{0} C_{p, q}\left\|\Gamma_{\mathcal{Q}}\right\|_{\ell_{w}^{q} \rightarrow \ell_{w}^{q}} \cdot\left(C^{\prime}\right)^{2}$. $\|Y\|_{\text {Schur }}\|Z\|_{\text {Schur }}$, as desired.

\section{Results for Structured Systems}

In this section, we provide further simplified conditions for the boundedness and invertibility of the frame operator. For this, we will assume throughout this section that the family $\left(g_{j}\right)_{j \in J}$ of functions $g_{j} \in L^{1}\left(\mathbb{R}^{d}\right) \cap L^{2}\left(\mathbb{R}^{d}\right)$ defining the system

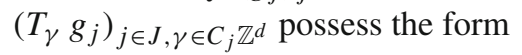

$$
g_{j}=\left|\operatorname{det} A_{j}\right|^{1 / 2} \cdot M_{b_{j}}\left[g \circ A_{j}^{t}\right]
$$

for certain $A_{j} \in \mathrm{GL}(d, \mathbb{R})$ and $b_{j} \in \widehat{\mathbb{R}}^{d}$ and a fixed $g \in L^{1}\left(\mathbb{R}^{d}\right) \cap L^{2}\left(\mathbb{R}^{d}\right)$ satisfying $\widehat{g} \in C^{\infty}\left(\widehat{\mathbb{R}}^{d}\right)$.

Observe that (7.1) can be written as $g_{j}=\left|\operatorname{det} A_{j}\right|^{-1 / 2} \cdot \mathcal{F}^{-1}\left(\widehat{g} \circ S_{j}^{-1}\right)$, where $S_{j}=A_{j}(\cdot)+b_{j}$.

\subsection{Simplified Criteria for Invertibility of the Frame Operator}

In this subsection, we give simplified versions of the estimates for the operator norms of $T_{0}^{-1}$ and $R_{0}$, under the assumption that the generators $\left(g_{j}\right)_{j \in J}$ of the sys- 
tem $\left(T_{\gamma} g_{j}\right)_{j \in J, \gamma \in C_{j} \mathbb{Z}^{d}}$ have the form (7.1) and that the lattices $C_{j} \mathbb{Z}^{d}$ are given by $C_{j}=\delta A_{j}^{-t}$ for a suitable $\delta>0$. We begin with a simplified version of Proposition 6.3.

Proposition 7.1 Let $\mathcal{Q}=\left(S_{j}\left(Q_{j}^{\prime}\right)\right)_{j \in J}=\left(A_{j}\left(Q_{j}^{\prime}\right)+b_{j}\right)_{j \in J}$ be an affinely generated cover of an open set $\mathcal{O} \subset \widehat{\mathbb{R}}^{d}$ of full measure. Let $\Phi=\left(\varphi_{j}\right)_{j \in J}$ be a regular partition of unity subordinate to $\mathcal{Q}$. Let $\left(T_{\gamma} g_{j}\right)_{j \in J, \gamma \in C_{j} \mathbb{Z}^{d}}$ be such that $C_{j}:=\delta \cdot A_{j}^{-t}$ for some $\delta>0$ and $g_{j}:=\left|\operatorname{det} A_{j}\right|^{1 / 2} \cdot M_{b_{j}}\left[g \circ A_{j}^{t}\right]$ for some $g \in L^{1}\left(\mathbb{R}^{d}\right) \cap L^{2}\left(\mathbb{R}^{d}\right)$ with $\widehat{g} \in C^{\infty}\left(\widehat{\mathbb{R}}^{d}\right)$. Suppose that there is some $A^{\prime}>0$ satisfying $A^{\prime} \leq \sum_{j \in J}\left|\widehat{g}\left(S_{j}^{-1} \xi\right)\right|^{2}$ for almost all $\xi \in \mathcal{O}$, and that

$$
\begin{aligned}
M_{0} & \left.:=\sup _{i \in J} \sum_{j \in J}\left[\left.\max \left\{1,\left\|A_{j}^{-1} A_{i}\right\|^{d+1}\right\} \cdot\left(\int_{Q_{i}^{\prime}|\alpha| \leq d+1} \mid \max ^{\alpha} \widehat{g}\right)\left(S_{j}^{-1}\left(S_{i} \xi\right)\right)\right|^{2(d+1)} d \xi\right)^{1 /(d+1)}\right] \\
& <\infty
\end{aligned}
$$

Then the function $t_{0}$ defined in Eq. (5.2) is continuous on $\mathcal{O}$ and tame, and the estimate $A^{\prime} \leq \sum_{j \in J}\left|\widehat{g}\left(S_{j}^{-1} \xi\right)\right|^{2}$ holds for all $\xi \in \mathcal{O}$. Furthermore, for any $p, q \in$ $[1, \infty]$ and any $\mathcal{Q}$-moderate weight $w=\left(w_{j}\right)_{j \in J}$, the operator

$$
T_{0}:=\Phi_{t_{0}}: \mathcal{D}\left(\mathcal{Q}, L^{p}, \ell_{w}^{q}\right) \rightarrow \mathcal{D}\left(\mathcal{Q}, L^{p}, \ell_{w}^{q}\right)
$$

with $\Phi_{t_{0}}$ as defined in Proposition 5.7 is well-defined, bounded, and boundedly invertible, with

$$
\left\|T_{0}^{-1}\right\|_{\mathcal{D}\left(\mathcal{Q}, L^{p}, \ell_{w}^{q}\right) \rightarrow \mathcal{D}\left(\mathcal{Q}, L^{p}, \ell_{w}^{q}\right)} \leq C_{d}^{\prime} \cdot N_{\mathcal{Q}}^{2} C_{\Phi} \cdot\left[\max _{|\alpha| \leq d+1} C_{\mathcal{Q}, \Phi, \alpha}\right] \cdot\left(A^{\prime}\right)^{-1} \cdot\left(\frac{M_{0}}{A^{\prime}}\right)^{d+1} \cdot \delta^{d},
$$

where $C_{d}^{\prime}=C_{d} \cdot(2 d)^{(d+1)^{2}}$ with $C_{d}$ as in Eq. (6.5).

Proof We apply Proposition 6.3. For this, note that since $C_{j}=\delta \cdot A_{j}^{-t}$ and $\widehat{g_{j}}=$ $\left|\operatorname{det} A_{j}\right|^{-1 / 2} \cdot \widehat{g} \circ S_{j}^{-1}$, the $\mathcal{Q}$-localized version $g_{i, j}^{\natural}$ of $g_{j}$ defined in (6.1) satisfies $\mathcal{F} g_{i, j}^{\natural}=\widehat{g_{j}} \circ S_{i}=\left|\operatorname{det} A_{j}\right|^{-1 / 2} \cdot \widehat{g} \circ S_{j}^{-1} \circ S_{i}$ and, moreover, $\left|\operatorname{det} C_{j}\right|^{-1} \cdot\left|\mathcal{F} g_{i, j}^{\natural}\right|^{2}=$ $\delta^{-d} \cdot|\widehat{g}|^{2} \circ S_{j}^{-1} \circ S_{i}$. Leibniz rule entails the pointwise estimate

$$
\begin{gathered}
\left.\left|\partial^{\alpha}\right| \widehat{g}\right|^{2}(\xi)|=| \partial^{\alpha}(\widehat{g}(\xi) \cdot \bar{g}(\xi))\left|\leq \sum_{\beta \leq \alpha}\left(\begin{array}{c}
\alpha \\
\beta
\end{array}\right)\right| \partial^{\beta} \widehat{g}(\xi)|\cdot| \partial^{\alpha-\beta} \overline{\widehat{g}}(\xi) \mid \leq 2^{|\alpha|} \\
\left(\max _{|\alpha| \leq d+1}\left|\partial^{\alpha} \widehat{g}(\xi)\right|\right)^{2}
\end{gathered}
$$


for any $\alpha \in \mathbb{N}_{0}^{d}$ with $|\alpha| \leq d+1$. Since $S_{j}^{-1} S_{i}=A_{j}^{-1} A_{i}(\cdot)+A_{j}^{-1}\left(b_{i}-b_{j}\right)$, it follows by the chain rule as in Lemma A.3 that, for any $v \in \mathbb{N}_{0}^{d}$ with $|\nu| \leq d+1$,

$$
\begin{aligned}
\left.\left|\operatorname{det} C_{j}\right|^{-1}\left|\partial^{\nu}\right| \mathcal{F} g_{i, j}^{\natural}\right|^{2}(\xi) \mid & \leq \delta^{-d} d^{|\nu|}\left\|A_{j}^{-1} A_{i}\right\|^{|\nu|} \max _{|\alpha|=|\nu|}\left|\left(\partial^{\alpha}|\widehat{g}|^{2}\right)\left(S_{j}^{-1}\left(S_{i} \xi\right)\right)\right| \\
& \leq \delta^{-d}(2 d)^{d+1} \max \left\{1,\left\|A_{j}^{-1} A_{i}\right\|^{d+1}\right\} \\
& \left(\max _{|\alpha| \leq d+1}\left|\left(\partial^{\alpha} \widehat{g}\right)\left(S_{j}^{-1}\left(S_{i} \xi\right)\right)\right|\right)^{2}
\end{aligned}
$$

for $\xi \in \widehat{\mathbb{R}}^{d}$. Using this, we can estimate the constant $M$ from Proposition 6.3 as follows:

$$
\begin{aligned}
& M=\sup _{i \in J} \sum_{j \in J}\left(\left|\operatorname{det} C_{j}\right|^{-1} \cdot\left\|\left.\max _{|\nu| \leq d+1}\left|\partial^{v}\right| \mathcal{F} g_{i, j}^{\natural}\right|^{2} \mid\right\|_{L^{d+1}\left(Q_{i}^{\prime}\right)}\right) \\
& \leq \delta^{-d}(2 d)^{d+1} \cdot \sup _{i \in J} \sum_{j \in J}\left[\max \left\{1,\left\|A_{j}^{-1} A_{i}\right\|^{d+1}\right\}\right. \\
& \left.\cdot\left(\int_{Q_{i}^{\prime}|\alpha| \leq d+1} \max \left|\left(\partial^{\alpha} \widehat{g}\right)\left(S_{j}^{-1}\left(S_{i} \xi\right)\right)\right|^{2(d+1)} d \xi\right)^{1 /(d+1)}\right] \\
& =\delta^{-d}(2 d)^{d+1} \cdot M_{0} \text {, }
\end{aligned}
$$

with $M_{0}$ as defined in the statement of the current proposition.

By assumption, we have $A^{\prime} \leq \sum_{j \in J}\left|\widehat{g}\left(S_{j}^{-1} \xi\right)\right|^{2}$, and thus

$$
t_{0}(\xi)=\sum_{j \in J}\left|\operatorname{det} C_{j}\right|^{-1}\left|\widehat{g_{j}}(\xi)\right|^{2}=\delta^{-d} \cdot \sum_{j \in J}\left|\widehat{g}\left(S_{j}^{-1} \xi\right)\right|^{2} \geq A^{\prime} \cdot \delta^{-d}
$$

for almost all $\xi \in \mathcal{O}$ and hence for almost all $\xi \in \widehat{\mathbb{R}}^{d}$. Therefore, Proposition 6.3 shows that $t_{0}$ is continuous on $\mathcal{O}$ and tame, that the preceding estimate holds pointwise on $\mathcal{O}$, and that the operator $T_{0}: \mathcal{D}\left(\mathcal{Q}, L^{p}, \ell_{w}^{q}\right) \rightarrow \mathcal{D}\left(\mathcal{Q}, L^{p}, \ell_{w}^{q}\right)$ is well-defined, bounded, and boundedly invertible with

$$
\begin{aligned}
\left\|T_{0}^{-1}\right\|_{\mathcal{D}\left(\mathcal{Q}, L^{p}, \ell_{w}^{q}\right) \rightarrow \mathcal{D}\left(\mathcal{Q}, L^{p}, \ell_{w}^{q}\right) \leq} & (2 d)^{(d+1)^{2}} C_{d} \cdot N_{\mathcal{Q}}^{2} C_{\Phi} \cdot\left[\max _{|\alpha| \leq d+1} C_{\mathcal{Q}, \Phi, \alpha}\right] \cdot\left(A^{\prime}\right)^{-1} \\
& \cdot\left(\frac{M_{0}}{A^{\prime}}\right)^{d+1} \cdot \delta^{d}
\end{aligned}
$$

This completes the proof.

Our next aim is to present a simplified version of the technical Lemma 6.4. For this, we will use the following result whose proof we postpone to Appendix D.2. 
Lemma 7.2 Let $g \in C^{d+1}\left(\widehat{\mathbb{R}}^{d}\right)$ be such that there exists a function $\varrho: \widehat{\mathbb{R}}^{d} \rightarrow[0, \infty)$ satisfying $\left|\partial^{\alpha} g(\xi)\right| \leq \varrho(\xi) \cdot(1+|\xi|)^{-(d+1)}$ for all $\xi \in \widehat{\mathbb{R}}^{d}$ and $\alpha \in \mathbb{N}_{0}^{d}$ with $|\alpha| \leq d+1$. Then, setting

$$
h_{1}(\xi):=\left(1+|\xi|^{2}\right)^{(d+1) / 2} \cdot g(\xi), \quad h_{2}(\xi):=\left(1+|\xi|^{2}\right)^{-(d+1) / 2}
$$

we have $g=h_{1} \cdot h_{2}$ on $\widehat{\mathbb{R}}^{d}$. Furthermore, $h_{1}, h_{2} \in C^{d+1}\left(\widehat{\mathbb{R}}^{d}\right)$ satisfy the estimates

$$
\max _{|\alpha| \leq d+1}\left|\partial^{\alpha} h_{2}(\xi)\right| \leq C^{\prime} \cdot(1+|\xi|)^{-(d+1)}, \max _{|\alpha| \leq d+1}\left|\partial^{\alpha} h_{1}(\xi)\right| \leq C^{\prime} \cdot \varrho(\xi)
$$

for all $\xi \in \widehat{\mathbb{R}}^{d}$, where $C^{\prime}:=\left(12 \cdot(d+1)^{2}\right)^{d+1}$.

Proposition 7.3 Let $\mathcal{Q}=\left(S_{j}\left(Q_{j}^{\prime}\right)\right)_{j \in J}=\left(A_{j}\left(Q_{j}^{\prime}\right)+b_{j}\right)_{j \in J}$ be an affinely generated cover of an open set $\mathcal{O} \subset \widehat{\mathbb{R}}^{d}$ offull measure. Let $\Phi=\left(\varphi_{j}\right)_{j \in J}$ be a regular partition of unity subordinate to $\mathcal{Q}$, and let $w=\left(w_{j}\right)_{j \in J}$ be $\mathcal{Q}$-moderate. Let $\left(T_{\gamma} g_{j}\right)_{j \in J, \gamma \in C_{j} \mathbb{Z}^{d}}$ be such that $C_{j}:=\delta \cdot A_{j}^{-t}$ for some $\delta \in(0,1]$ and $g_{j}:=\left|\operatorname{det} A_{j}\right|^{1 / 2} \cdot M_{b_{j}}\left[g \circ A_{j}^{t}\right]$ for some $g \in L^{1}\left(\mathbb{R}^{d}\right) \cap L^{2}\left(\mathbb{R}^{d}\right)$ satisfying $\widehat{g} \in C^{\infty}\left(\widehat{\mathbb{R}}^{d}\right)$. Assume that the function $t_{0}$ defined in Eq. (5.2) is tame. Assume that $\widetilde{Y}=\left(\widetilde{Y}_{i, j}\right)_{i, j \in J}$ is of Schur-type, where

$$
\tilde{Y}_{i, j}:=K_{i, j} \cdot \int_{Q_{i}^{\prime}}\left(1+\left|S_{j}^{-1}\left(S_{i} \xi\right)\right|\right)^{d+1} \max _{|\alpha| \leq d+1}\left|\left[\partial^{\alpha} \widehat{g}\right]\left(S_{j}^{-1}\left(S_{i} \xi\right)\right)\right| d \xi
$$

with

$$
\begin{aligned}
K_{i, j}:= & \max \left\{\frac{w_{i}}{w_{j}}, \frac{w_{j}}{w_{i}}\right\}\left(\max \left\{1,\left|A_{i}^{-1}\left(b_{i}-b_{j}\right)\right|\right\} \max \left\{1,\left\|A_{i}^{-1} A_{j}\right\|\right\}\right. \\
& \left.\max \left\{1,\left\|A_{j}^{-1} A_{i}\right\|^{2}\right\}\right)^{d+1} .
\end{aligned}
$$

Then the system $\left(T_{\gamma} g_{j}\right)_{j \in J, \gamma \in C_{j} \mathbb{Z}^{d}}$ is $(w, w, \Phi)$-adapted. Furthermore, for any $p, q \in$ $[1, \infty]$, the operator $R_{0}: \mathcal{D}\left(\mathcal{Q}, L^{p}, \ell_{w}^{q}\right) \rightarrow \mathcal{D}\left(\mathcal{Q}, L^{p}, \ell_{w}^{q}\right)$ defined in Corollary 5.9 is well-defined and bounded, with

$$
\left\|R_{0}\right\|_{\mathcal{D}\left(\mathcal{Q}, L^{p}, \ell_{w}^{q}\right) \rightarrow \mathcal{D}\left(\mathcal{Q}, L^{p}, \ell_{w}^{q}\right)} \leq C_{0} C_{p, q}\left(C^{\prime}\right)^{4}\left\|\Gamma_{\mathcal{Q}}\right\|_{\ell_{w}^{q} \rightarrow \ell_{w}^{q}} \cdot \delta^{2} \cdot\|\widetilde{Y}\|_{\text {Schur }}^{2}
$$

with $C_{0}$ as in (6.9), $C^{\prime}$ as in Lemma 7.2 and $C_{p, q}:=1$ if $\max \{p, q\}<\infty$ and $C_{p, q}:=C_{\Phi}\left\|\Gamma_{\mathcal{Q}}\right\|_{\ell_{w}^{q} \rightarrow \ell_{w}^{q}}^{2}$, otherwise.

Proof To show that $\left(T_{\gamma} g_{j}\right)_{j \in J, \gamma \in C_{j} \mathbb{Z}^{d}}$ is $(w, w, \Phi)$-adapted, we use Proposition 6.2. Let us set $v_{j}:=w_{j}$ for $j \in J$. Note that $\mathcal{F} g_{i, j}^{\natural}=\widehat{g_{j}} \circ S_{i}=\left|\operatorname{det} A_{j}\right|^{-1 / 2} \cdot \widehat{g} \circ S_{j}^{-1} \circ S_{i}$. An application of the chain rule as in Lemma A.3 shows, for any $\alpha \in \mathbb{N}_{0}^{d}$ with 
$|\alpha| \leq d+1$, that

$$
\begin{aligned}
\left|\partial^{\alpha}\left[\mathcal{F} g_{i, j}^{\natural}\right](\xi)\right| \leq & \left|\operatorname{det} A_{j}\right|^{-1 / 2} \cdot d^{|\alpha|}\left\|A_{j}^{-1} A_{i}\right\|^{|\alpha|} \max _{|\beta|=|\alpha|}\left|\left(\partial^{\beta} \widehat{g}\right)\left(S_{j}^{-1}\left(S_{i} \xi\right)\right)\right| \\
\leq & \left|\operatorname{det} A_{j}\right|^{-1 / 2} \cdot d^{d+1} \max \left\{1,\left\|A_{j}^{-1} A_{i}\right\|^{d+1}\right\}\left(1+\left|S_{j}^{-1}\left(S_{i} \xi\right)\right|\right)^{d+1} \\
& \cdot \max _{|\alpha| \leq d+1}\left|\left(\partial^{\alpha} \widehat{g}\right)\left(S_{j}^{-1}\left(S_{i} \xi\right)\right)\right|,
\end{aligned}
$$

and hence $\int_{Q_{i}^{\prime}} \max _{|\alpha| \leq d+1}\left|\partial^{\alpha}\left[\mathcal{F} g_{i, j}^{\natural}\right](\xi)\right| d \xi \leq\left|\operatorname{det} A_{j}\right|^{-1 / 2} \cdot d^{d+1} \max \{1$, $\left.\left\|A_{j}^{-1} A_{i}\right\|^{d+1}\right\} \cdot \widetilde{Y}_{i, j} K_{i, j}^{-1}$. Thus, the matrix entries $G_{i, j}$ introduced in Proposition 6.2 satisfy

$$
\begin{aligned}
G_{i, j} & \leq \delta^{-d / 2} d^{d+1} \max \left\{\frac{w_{i}}{w_{j}}, \frac{w_{j}}{w_{i}}\right\} \max \left\{1,\left\|A_{j}^{-1} A_{i}\right\|^{d+1}\right\}\left(1+\delta\left\|A_{j}^{-1} A_{i}\right\|\right)^{d} \frac{\widetilde{Y}_{i, j}}{K_{i, j}} \\
& \leq C_{d, \delta} \cdot \widetilde{Y}_{i, j},
\end{aligned}
$$

for a suitable constant $C_{d, \delta}>0$ which is independent of $i, j \in J$. Thus $\|G\|_{\text {Schur }}<\infty$.

To finish the proof, we will show the claimed bound on $\left\|R_{0}\right\|_{\mathcal{D}\left(\mathcal{Q}, L^{p}, \ell_{w}^{q}\right) \rightarrow \mathcal{D}\left(\mathcal{Q}, L^{p}, \ell_{w}^{q}\right)}$. For this, we will apply Lemma 6.4 with the choices $I=J, B_{j} \stackrel{=}{=} A_{j}, c_{j}=b_{j}$ and $v_{j}=w_{j}$. In this setting, we have $g_{j}^{\diamond}=g$ for all $j \in J$. By defining $\varrho: \widehat{\mathbb{R}}^{d} \rightarrow[0, \infty), \quad \xi \mapsto(1+|\xi|)^{d+1} \max _{|\alpha| \leq d+1}\left|\partial^{\alpha} \widehat{g}(\xi)\right|$, we clearly have $\left|\partial^{\alpha} \widehat{g}(\xi)\right| \leq \varrho(\xi) \cdot(1+|\xi|)^{-(d+1)}$ for all $\xi \in \widehat{\mathbb{R}}^{d}$ and $\alpha \in \mathbb{N}_{0}^{d}$ with $|\alpha| \leq d+1$. Hence, by Lemma 7.2, we can factorize $\widehat{g}=h_{1} \cdot h_{2}$ with $h_{1}, h_{2} \in C^{d+1}\left(\widehat{\mathbb{R}}^{d}\right)$ satisfying (7.2). This shows that the first hypothesis in Lemma 6.4 is satisfied, and it remains to show that the matrices $Y=\left(Y_{i, j}\right)_{i, j \in J}$ and $Z=\left(Z_{i, j}\right)_{i, j \in J}$ of Lemma 6.4 are of Schur-type. For this, note that $\left|\operatorname{det}\left(B_{j}^{t} C_{j}\right)\right|^{-1}=\left|\operatorname{det}\left(A_{j}^{t} \delta A_{j}^{-t}\right)\right|^{-1}=\delta^{-d}$ and $\left\|C_{j}^{t} A_{i}\right\|=\delta\left\|A_{j}^{-1} A_{i}\right\| \leq\left\|A_{j}^{-1} A_{i}\right\|$, since $\delta \leq 1$. Therefore,

$$
\begin{array}{r}
\max \left\{\left\|C_{j}^{t} A_{i}\right\|,\left\|C_{j}^{t} A_{i}\right\|^{d+1}\right\} \leq q \delta\left\|A_{j}^{-1} A_{i}\right\| \cdot \max \left\{1,\left\|A_{j}^{-1} A_{i}\right\|^{d}\right\} \\
\leq \delta \max \left\{1,\left\|A_{j}^{-1} A_{i}\right\|^{d+1}\right\}
\end{array}
$$

for all $i, j \in I$. It is now readily verified that $Y_{i, j} \leq C^{\prime} \cdot \delta \cdot \widetilde{Y}_{i, j}$ and $Z_{i, j} \leq C^{\prime} \cdot \delta \cdot \widetilde{Y}_{i, j}$ for $i, j \in J$, where $C^{\prime}$ is as in Lemma 7.2. Hence, $\|Y\|_{\text {Schur }}\|Z\|_{\text {Schur }} \leq\left(C^{\prime}\right)^{2} \cdot \delta^{2} \cdot\|\widetilde{Y}\|_{\text {Schur }}^{2}$. Therefore, applying Lemma 6.4 completes the proof.

The factor $\max \left\{1,\left|A_{i}^{-1}\left(b_{i}-b_{j}\right)\right|\right\}$ that appears in defining $K_{i, j}$ in Proposition 7.3 can be inconvenient. In particular, it does not appear in [62], which makes it difficult to translate existing concrete examples from [62] readily to the present setting. For this reason, we supply the following.

Lemma 7.4 The matrix entries $\widetilde{Y}_{i, j}$ introduced in Proposition 7.3 satisfy $0 \leq \widetilde{Y}_{i, j} \leq$ $\left(1+R_{\mathcal{Q}}\right)^{d+1} \cdot \widehat{Y}_{i, j}$, where

$$
\widehat{Y}_{i, j}:=L_{i, j} \cdot \int_{Q_{i}^{\prime}}\left(1+\left|S_{j}^{-1}\left(S_{i} \xi\right)\right|\right)^{2 d+2} \max _{|\alpha| \leq d+1}\left|\left(\partial^{\alpha} \widehat{g}\right)\left(S_{j}^{-1}\left(S_{i} \xi\right)\right)\right| d \xi
$$


and $L_{i, j}:=\max \left\{\frac{w_{i}}{w_{j}}, \frac{w_{j}}{w_{i}}\right\}\left(\max \left\{1,\left\|A_{i}^{-1} A_{j}\right\|^{2}\right\} \max \left\{1,\left\|A_{j}^{-1} A_{i}\right\|^{3}\right\}\right)^{d+1}$ for $i, j \in$ $J$.

Proof Since $S_{j}^{-1}\left(S_{i} \xi\right)=A_{j}^{-1}\left(A_{i} \xi+b_{i}-b_{j}\right)$ for all $\xi \in \widehat{\mathbb{R}}^{d}$, it follows that

$$
\begin{aligned}
\left|A_{i}^{-1}\left(b_{i}-b_{j}\right)\right|= & \left|A_{i}^{-1} A_{j} A_{j}^{-1}\left(b_{i}-b_{j}\right)\right| \leq\left\|A_{i}^{-1} A_{j}\right\| \cdot\left(\left|A_{j}^{-1} A_{i} \xi+A_{j}^{-1}\left(b_{i}-b_{j}\right)\right|\right. \\
& \left.+\left|A_{j}^{-1} A_{i} \xi\right|\right) \\
\leq & \left\|A_{i}^{-1} A_{j}\right\| \cdot\left(\left|S_{j}^{-1}\left(S_{i} \xi\right)\right|+R_{\mathcal{Q}}\left\|A_{j}^{-1} A_{i}\right\|\right) \\
\leq & \left(1+R_{\mathcal{Q}}\right) \cdot \max \left\{1,\left\|A_{i}^{-1} A_{j}\right\|\right\} \cdot \max \left\{1,\left\|A_{j}^{-1} A_{i}\right\|\right\} \cdot\left(1+\left|S_{j}^{-1}\left(S_{i} \xi\right)\right|\right)
\end{aligned}
$$

for $\xi \in Q_{i}^{\prime}$. Using this, the estimate $\widetilde{Y}_{i, j} \leq\left(1+R_{\mathcal{Q}}\right)^{d+1} \cdot \widehat{Y}_{i, j}$ follows directly from the definitions.

\subsection{Invertibility of the Frame Operator}

The next result summarizes our criteria for the invertibility of the frame operator obtained in this section.

Theorem 7.5 Let $\mathcal{Q}=\left(S_{j}\left(Q_{j}^{\prime}\right)\right)_{j \in J}=\left(A_{j}\left(Q_{j}^{\prime}\right)+b_{j}\right)_{j \in J}$ be an affinely generated cover of an open set $\mathcal{O} \subset \widehat{\mathbb{R}}^{d}$ of full measure. Let $\Phi=\left(\varphi_{j}\right)_{j \in J}$ be a regular partition of unity subordinate to $\mathcal{Q}$, and let $w=\left(w_{j}\right)_{j \in J}$ be $\mathcal{Q}$-moderate. Suppose that

(i) The system $\left(T_{\gamma} g_{j}\right)_{j \in J, \gamma \in C_{j} \mathbb{Z}^{d}}$ is such that $g_{j}:=\left|\operatorname{det} A_{j}\right|^{1 / 2} \cdot M_{b_{j}}\left[g \circ A_{j}^{t}\right]$ and $C_{j}:=\delta \cdot A_{j}^{-t}$ for some $\delta>0$ and some $g \in L^{1}\left(\mathbb{R}^{d}\right) \cap L^{\infty}\left(\mathbb{R}^{d}\right)$ with $\widehat{g} \in C^{\infty}\left(\widehat{\mathbb{R}}^{d}\right) ;$

(ii) There is an $A^{\prime}>0$ such that $A^{\prime} \leq \sum_{j \in J}\left|\widehat{g}\left(S_{j}^{-1} \xi\right)\right|^{2}$ for almost all $\xi \in \mathcal{O}$;

(iii) The matrix $\widehat{Y}=\left(\widehat{Y}_{i, j}\right)_{i, j \in J}$ is of Schur-type, where $\widehat{Y}_{i, j}$ as in Lemma 7.4;

(iv) The term $M_{0}$ defined in Proposition 7.1 is finite.

Then the system $\left(T_{\gamma} g_{j}\right)_{j \in J, \gamma \in C_{j} \mathbb{Z}^{d}}$ is $(w, w, \Phi)$-adapted, and for $p, q \in[1, \infty]$, the frame operator $S: \mathcal{D}\left(\mathcal{Q}, L^{p}, \ell_{w}^{q}\right) \rightarrow \mathcal{D}\left(\mathcal{Q}, L^{p}, \ell_{w}^{q}\right)$ associated to $\left(T_{\gamma} g_{j}\right)_{j \in J, \gamma \in C_{j} \mathbb{Z}^{d}}$ is well-defined and bounded.

Finally, for given $p, q \in[1, \infty]$, let $C_{d, \mathcal{Q}, w}:=\max \left\{\left[\sup _{j \in J} \lambda\left(Q_{j}^{\prime}\right)\right]^{-\frac{3}{d+2}}\right.$, $\left.\left[\kappa_{d} K_{\mathcal{Q}, w}\right]^{1 /(d+2)}\right\}$, where

$$
\begin{aligned}
\kappa_{d}:= & (2 d)^{(d+1)^{2}}(8 d)^{2 d+2} 12^{5 d+5} \cdot(d+1)^{8 d+10} \cdot \frac{72 \cdot(d+1)^{5 / 2} \cdot 2^{d+2}}{\pi^{3 d}} . \\
& \left(\frac{\frac{0.8}{e}(d+1)^{2}}{\ln (2+d)}\right)^{d+1}
\end{aligned}
$$

and $K_{\mathcal{Q}, w}:=\left\|\Gamma_{\mathcal{Q}}\right\|_{\ell_{w}^{q} \rightarrow \ell_{w}^{q}}^{3} N_{\mathcal{Q}}^{2} \max \left\{1, C_{\Phi}^{2}\right\}\left(1+R_{\mathcal{Q}}\right)^{3 d+4} \max _{|\alpha| \leq d+1} C_{\mathcal{Q}, \Phi, \alpha}^{3}$. Then, if $\delta>0$ is chosen such that

$$
C_{d, \mathcal{Q}, w} \cdot M_{0}^{\frac{d+1}{d+2}} \cdot\left(\|\widehat{Y}\|_{\text {Schur }}^{2}\right)^{\frac{1}{d+2}} \cdot \frac{\delta}{A^{\prime}}<1
$$


then the frame operator is also boundedly invertible as an operator on $\mathcal{D}\left(\mathcal{Q}, L^{p}, \ell_{w}^{q}\right)$.

Proof We proceed in two steps.

Step 1. Suppose that $\delta \leq 1$. Since $A^{\prime} \leq \sum_{j \in J}\left|\widehat{g}\left(S_{j}^{-1} \xi\right)\right|^{2}$ for almost all $\xi \in \mathcal{O}$, and since $M_{0}$ is finite, an application of Proposition 7.1 shows that $t_{0}$ is continuous on $\mathcal{O}$ and tame and that $T_{0}:=\Phi_{t_{0}}: \mathcal{D}\left(\mathcal{Q}, L^{p}, \ell_{w}^{q}\right) \rightarrow \mathcal{D}\left(\mathcal{Q}, L^{p}, \ell_{w}^{q}\right)$, with $\Phi_{t_{0}}$ as defined in Proposition 5.7, is well-defined, bounded, and boundedly invertible, with

$$
\left\|T_{0}^{-1}\right\|_{\mathcal{D}\left(\mathcal{Q}, L^{p}, \ell_{w}^{q}\right) \rightarrow \mathcal{D}\left(\mathcal{Q}, L^{p}, \ell_{w}^{q}\right)} \leq C^{(1)} \cdot M_{0}^{d+1} \cdot\left(A^{\prime}\right)^{-(d+2)} \cdot \delta^{d}
$$

for arbitrary $p, q \in[1, \infty]$. Here, $C^{(1)}:=(2 d)^{(d+1)^{2}} C_{d} N_{\mathcal{Q}}^{2} C_{\Phi} \cdot \max _{|\alpha| \leq d+1} C_{\mathcal{Q}, \Phi, \alpha}$, with $C_{d}$ as in Eq. (6.5).

Lemma 7.4 shows that $\|\widetilde{Y}\|_{\text {Schur }} \leq\left(1+R_{\mathcal{Q}}\right)^{d+1}\|\widehat{Y}\|_{\text {Schur }}<\infty$, with $\tilde{Y}$ as in Proposition 7.3. Therefore, Proposition 7.3 shows that the system $\left(T_{\gamma} g_{j}\right)_{j \in J, \gamma \in C_{j} \mathbb{Z}^{d}}$ is $(w, w, \Phi)$-adapted, and hence the frame operator $S: \mathcal{D}\left(\mathcal{Q}, L^{p}, \ell_{w}^{q}\right) \rightarrow \mathcal{D}\left(\mathcal{Q}, L^{p}, \ell_{w}^{q}\right)$ is well-defined and bounded for all $p, q \in[1, \infty]$ by Corollary 4.10 .

Lastly, it follows by Proposition 7.3 and Corollary 5.9 that the frame operator $S$ can be written as $S=T_{0}+R_{0}$, where

$\left\|R_{0}\right\|_{\mathcal{D}\left(\mathcal{Q}, L^{p}, \ell_{w}^{q}\right) \rightarrow \mathcal{D}\left(\mathcal{Q}, L^{p}, \ell_{w}^{q}\right)} \leq C^{(2)} \cdot \delta^{2} \cdot\|\widetilde{Y}\|_{\text {Schur }}^{2} \leq C^{(2)}\left(1+R_{\mathcal{Q}}\right)^{2 d+2} \cdot \delta^{2} \cdot\|\widehat{Y}\|_{\text {Schur }}^{2}$,

where $C^{(2)}:=C_{0} C_{p, q}\left(C^{\prime}\right)^{4}\left\|\Gamma_{\mathcal{Q}}\right\|_{\ell_{w}^{q} \rightarrow \ell_{w}^{q}}$, with $C_{0}$ as in (6.9) and $C^{\prime}$ as in Lemma 7.2, and with $C_{p, q}:=\max \left\{1, C_{\Phi}\right\} \cdot\left\|\Gamma_{\mathcal{Q}}\right\|_{\ell_{w}^{q} \rightarrow \ell_{w}^{q}}^{2}$. Here, we used the easily verifiable estimate $\left\|\Gamma_{\mathcal{Q}}\right\|_{\ell_{w}^{q} \rightarrow \ell_{w}^{q}} \geq 1$.

Therefore, for arbitrary $p, q \in[1, \infty]$, a combination of the above estimates gives

$$
\begin{aligned}
\left\|T_{0}^{-1}\right\|_{\mathrm{op}} \cdot\left\|R_{0}\right\|_{\mathrm{op}} & \leq C^{(1)} C^{(2)}\left(1+R_{\mathcal{Q}}\right)^{2 d+2} \cdot \delta^{2+d} \cdot\|\widehat{Y}\|_{\text {Schur }}^{2} \cdot M_{0}^{d+1} \cdot\left(A^{\prime}\right)^{-(d+2)} \\
& =\left[\left(C^{(1)} C^{(2)}\left(1+R_{\mathcal{Q}}\right)^{2 d+2}\right)^{1 /(d+2)} \cdot M_{0}^{\frac{d+1}{d+2}} \cdot\left(\|\widehat{Y}\|_{\text {Schur }}^{2}\right)^{\frac{1}{d+2}} \cdot \frac{\delta}{A^{\prime}}\right]^{d+2} \\
& \leq\left[C_{d, \mathcal{Q}, w} \cdot M_{0}^{\frac{d+1}{d+2}} \cdot\left(\|\widehat{Y}\|_{\text {Schur }}^{2}\right)^{\frac{1}{d+2}} \cdot \frac{\delta}{A^{\prime}}\right]^{d+2}<1 .
\end{aligned}
$$

Therefore, Lemma 5.4 implies that the frame operator $S=T_{0}+R_{0}$ : $\mathcal{D}\left(\mathcal{Q}, L^{p}, \ell_{w}^{q}\right) \rightarrow \mathcal{D}\left(\mathcal{Q}, L^{p}, \ell_{w}^{q}\right)$ is boundedly invertible, as claimed.

Step 2. In this step it will be shown that (7.3) already entails $\delta \leq 1$. To this end, first note that $A^{\prime} \leq \sum_{j \in J}\left|\widehat{g}\left(S_{j}^{-1} \eta\right)\right|^{2} \leq\left(\sum_{j \in J}\left|\widehat{g}\left(S_{j}^{-1} \eta\right)\right|\right)^{2}$, and hence $\sum_{j \in J}\left|\widehat{g}\left(S_{j}^{-1} \eta\right)\right| \geq$ $\sqrt{A^{\prime}}$ for almost every $\eta \in \mathcal{O}$. Thus, for any fixed $i \in J$,

$$
\|\widehat{Y}\|_{\text {Schur }} \geq \sum_{j \in J} \widehat{Y}_{i, j} \geq \int_{Q_{i}^{\prime}} \sum_{j \in J}\left|\widehat{g}\left(S_{j}^{-1}\left(S_{i} \xi\right)\right)\right| d \xi \geq \int_{Q_{i}^{\prime}} \sqrt{A^{\prime}} d \xi=\sqrt{A^{\prime}} \cdot \lambda\left(Q_{i}^{\prime}\right) .
$$


Next, by applying Jensen's inequality, we see that the constant $M_{0}$ introduced in Proposition 7.1 satisfies, for each $i \in J$, the estimate

$$
\begin{aligned}
M_{0} & \geq \sum_{j \in J}\left(\lambda\left(Q_{i}^{\prime}\right) \int_{Q_{i}^{\prime}}\left|\widehat{g}\left(S_{j}^{-1}\left(S_{i} \xi\right)\right)\right|^{2(d+1)} \frac{d \xi}{\lambda\left(Q_{i}^{\prime}\right)}\right)^{1 /(d+1)} \\
& \geq\left[\lambda\left(Q_{i}^{\prime}\right)\right]^{1 /(d+1)-1} \sum_{j \in J} \int_{Q_{i}^{\prime}}\left|\widehat{g}\left(S_{j}^{-1}\left(S_{i} \xi\right)\right)\right|^{2} d \xi \\
& =\left[\lambda\left(Q_{i}^{\prime}\right)\right]^{1 /(d+1)-1} \int_{Q_{i}^{\prime}} \sum_{j \in J}\left|\widehat{g}\left(S_{j}^{-1}\left(S_{i} \xi\right)\right)\right|^{2} d \xi \\
& \geq\left[\lambda\left(Q_{i}^{\prime}\right)\right]^{1 /(d+1)-1} \cdot A^{\prime} \cdot \lambda\left(Q_{i}^{\prime}\right) \\
& =A^{\prime} \cdot\left[\lambda\left(Q_{i}^{\prime}\right)\right]^{1 /(d+1)} .
\end{aligned}
$$

Overall, we see that

$$
\kappa:=M_{0}^{\frac{d+1}{d+2}} \cdot\left(\|\widehat{Y}\|_{\text {Schur }}^{2}\right)^{\frac{1}{d+2}} \geq A^{\prime} \cdot \sup _{i \in J}\left[\lambda\left(Q_{i}^{\prime}\right)\right]^{\frac{3}{d+2}} \geq C_{d, \mathcal{Q}, w}^{-1} A^{\prime}
$$

and hence $C_{d, \mathcal{Q}, w} \cdot \kappa \cdot \frac{\delta}{A^{\prime}} \geq \delta$. Thus, if $\delta$ satisfies Eq. (7.3), then $\delta<1$.

\subsection{Proof of Theorem 1.1}

Theorem 1.1, announced in the introduction, is just a reformulation of Theorem 7.5, with the following identifications of notation: $\mathrm{A}=A^{\prime} ; \mathrm{B}=B^{\prime} ; M_{1}=\|\widehat{Y}\|_{\text {Schur }}$.

\subsection{Banach Frames and Atomic Decompositions}

We now remark that, under the assumptions of Theorem 7.5, the system $\left(T_{\delta A_{j}^{-t} k} g_{j}\right)_{j \in J, k \in \mathbb{Z}^{d}}$ forms a Banach frame and an atomic decomposition ([33]) for the Besov-type spaces $\mathcal{D}\left(\mathcal{Q}, L^{p}, \ell_{w}^{q}\right)$, and, moreover, the corresponding dual family is given by the canonical dual frame.

Corollary 7.6 Suppose that the assumptions of Theorem 7.5 are satisfied, including the assumption (7.3). Then the system $\left(T_{\delta A_{j}^{-t} k} g_{j}\right)_{j \in J, k \in \mathbb{Z}^{d}}$ forms a Banach frame and an atomic decomposition for all of the spaces $\mathcal{D}\left(\mathcal{Q}, L^{p}, \ell_{w}^{q}\right), p, q \in[1, \infty]$, with associated coefficient space $Y_{w}^{p, q}$ as in Definition 4.5. Precisely, the analysis and synthesis maps

$$
\begin{gathered}
\mathscr{C}: \mathcal{D}\left(\mathcal{Q}, L^{p}, \ell_{w}^{q}\right) \rightarrow Y_{w}^{p, q}, f \mapsto\left(\left\langle f \mid T_{\delta A_{j}^{-t} k} g_{j}\right\rangle_{\Phi}\right)_{j \in J, k \in \mathbb{Z}^{d}} \\
\text { and } \mathscr{D}: Y_{w}^{p, q} \rightarrow \mathcal{D}\left(\mathcal{Q}, L^{p}, \ell_{w}^{q}\right),\left(c_{j}^{(k)}\right)_{j \in J, k \in \mathbb{Z}^{d}} \mapsto \sum_{j \in J} \sum_{k \in \mathbb{Z}^{d}} c_{j}^{(k)} T_{\delta A_{j}^{-t} k} g_{j}
\end{gathered}
$$


are well-defined and bounded, and satisfy

$$
\left(S^{-1} \circ \mathscr{D}\right) \circ \mathscr{C}=\mathrm{id}_{\mathcal{D}\left(\mathcal{Q}, L^{p}, \ell_{w}^{q}\right)} \text { and } \mathscr{D} \circ\left(\mathscr{C} \circ S^{-1}\right)=\operatorname{id}_{\mathcal{D}\left(\mathcal{Q}, L^{p}, \ell_{w}^{q}\right)}
$$

Proof Theorem 7.5 shows that $\left(T_{\delta A_{j}^{-t} k} g_{j}\right)_{j \in J, k \in \mathbb{Z}^{d}}$ is $(w, w, \Phi)$-adapted. Thus, the boundedness of $\mathscr{C}, \mathscr{D}$ follows from Proposition 4.8. The remaining statements follow from the invertibility of $S=\mathscr{D} \circ \mathscr{C}$ proven in Theorem 7.5.

\subsection{An Example}

We conclude with an example verifying the hypotheses of Theorem 7.5 for Besovtype spaces associated with covers that have a geometry which is in a certain sense intermediate between the geometry of the uniform and the dyadic covers. These covers are an instance of the non-homogeneous isotropic covers from [56,Sect. 2.5] and [58,Sect. 2.1]; the corresponding spaces are also known as $\alpha$-modulation spaces [32]. For similar calculations of other concrete examples, we refer to [62].

For fixed $\alpha \in[0,1)$, the $\alpha$-modulation space with parameters $p, q \in[1, \infty]$ and $s \in \mathbb{R}$ is defined as $M_{p, q}^{s, \alpha}\left(\mathbb{R}^{d}\right):=\mathcal{D}\left(\mathcal{Q}^{(\alpha)}, L^{p}, \ell_{w^{(s, \alpha)}}^{q}\right)$, where the cover $\mathcal{Q}^{(\alpha)}$ of $\widehat{\mathbb{R}}^{d}$ is given by

$$
\mathcal{Q}^{(\alpha)}:=\left(A_{j}^{(\alpha)} Q+b_{j}^{(\alpha)}\right)_{j \in \mathbb{Z}^{d} \backslash\{0\}},
$$

where $A_{j}^{(\alpha)}:=|j|^{\alpha_{0}} \operatorname{id}_{\mathbb{R}^{d}}, \quad b_{j}^{(\alpha)}:=|j|^{\alpha_{0}} j$, and $Q=B_{r}(0)$, with $\alpha_{0}:=\frac{\alpha}{1-\alpha}$ and $r \geq r_{0}=r_{0}(d, \alpha)$. Under this assumption on $r$, one can show that $\mathcal{Q}^{(\alpha)}$ is indeed an affinely generated cover of $\widehat{\mathbb{R}}^{d}$; see [10,Theorem 2.6] and [62,Lemma 7.3]. Finally, the weight $w^{(s, \alpha)}$ is given by $w_{j}^{(s, \alpha)}=|j|^{s /(1-\alpha)}$ for $j \in \mathbb{Z}^{d} \backslash\{0\}$. In the following, we will simply write $\mathcal{Q}, A_{j}$, and $b_{j}$ for $\mathcal{Q}^{(\alpha)}, A_{j}^{(\alpha)}$, and $b_{j}^{(\alpha)}$ and fix some $r \geq r_{0}(d, \alpha)$.

Fix $s_{0} \geq 0$. In the following, we will only consider "smoothness parameters" $s \in\left[-s_{0}, s_{0}\right]$. Take $g \in L^{1}\left(\mathbb{R}^{d}\right) \cap L^{2}\left(\mathbb{R}^{d}\right)$ such that $\widehat{g} \in C^{\infty}\left(\widehat{\mathbb{R}}^{d}\right)$, and assume that there are $c, C>0$ and $N>0$ such that

$$
|\widehat{g}(\xi)| \geq c \quad \forall|\xi| \leq r \quad \text { and } \quad \max _{|\alpha| \leq d+1}\left|\partial^{\alpha} \widehat{g}(\xi)\right| \leq C \cdot(1+|\xi|)^{-N} \quad \forall \xi \in \widehat{\mathbb{R}}^{d}
$$

We will determine conditions on $N$ (depending on $d, \alpha, s_{0}$ ) which ensure that the prerequisites of Theorem 7.5 are satisfied. In fact, it will turn out that it is enough if $N>4 d+3+\tau$ where $\tau:=\frac{4 \alpha d+3 \alpha+s_{0}}{1-\alpha} \in[0, \infty)$.

To show this, note because of $Q_{i}^{\prime}=B_{r}(0)$ for all $i \in \mathbb{Z}^{d} \backslash\{0\}$ that

$$
S_{j}^{-1}\left(S_{i} Q_{i}^{\prime}\right)=B_{R_{i, j}}\left(\xi_{i, j}\right) \text { where } R_{i, j}=(|i| /|j|)^{\alpha_{0}} \cdot r \quad \text { and } \quad \xi_{i, j}=(|i| /|j|)^{\alpha_{0}} \cdot i-j .
$$


Thus, applying the change of variables $\eta=S_{j}^{-1}\left(S_{i} \xi\right)$, combined with the estimate (7.4), yields

$$
\begin{aligned}
Z_{i, j} & :=\int_{Q_{i}^{\prime}}\left(1+\left|S_{j}^{-1}\left(S_{i} \xi\right)\right|\right)^{2 d+2} \max _{|\alpha| \leq d+1}\left|\left[\partial^{\alpha} \widehat{g}\right]\left(S_{j}^{-1}\left(S_{i} \xi\right)\right)\right| d \xi \\
& =\left(\frac{|j|}{|i|}\right)^{d \alpha_{0}} \int_{B_{R_{i, j}}\left(\xi_{i, j}\right)}(1+|\eta|)^{2 d+2} \max _{|\alpha| \leq d+1}\left|\left[\partial^{\alpha} \widehat{g}\right](\eta)\right| d \eta \\
& \leq C\left(\frac{|j|}{|i|}\right)^{d \alpha_{0}} \int_{B_{R_{i, j}}\left(\xi_{i, j}\right)}(1+|\eta|)^{2 d+2-N} d \eta
\end{aligned}
$$

A similar computation shows

$$
\begin{aligned}
W_{i, j} & :=\left(\int_{Q_{i}^{\prime}} \max _{|\alpha| \leq d+1}\left|\left(\partial^{\alpha} \widehat{g}\right)\left(S_{j}^{-1}\left(S_{i} \xi\right)\right)\right|^{2(d+1)} d \xi\right)^{\frac{1}{d+1}} \\
& \leq C^{2}\left(\left(\frac{|j|}{|i|}\right)^{d \alpha_{0}} \int_{B_{R_{i, j}}\left(\xi_{i, j}\right)}(1+|\eta|)^{-2 N(d+1)} d \eta\right)^{\frac{1}{d+1}} .
\end{aligned}
$$

Using the notations

$$
\Lambda_{i, j}^{[M, \tau]}:=\left(\int_{B_{R_{i, j}}\left(\xi_{i, j}\right)}(1+|\eta|)^{-M} d \eta\right)^{\tau} \quad \text { and } \quad \Xi_{i, j}^{[k, M, \tau]}:=\left(\frac{|j|}{|i|}\right)^{k} \cdot \Lambda_{i, j}^{[M, \tau]}
$$

for $i, j \in \mathbb{Z}^{d} \backslash\{0\}$ and $k, M \in \mathbb{R}, \tau \in(0, \infty)$, we have thus shown

$$
Z_{i, j} \leq C \cdot \Xi_{i, j}^{\left[d \alpha_{0}, N-2 d-2,1\right]} \text { and } \quad W_{i, j} \leq C^{2} \cdot \Xi_{i, j}^{\left[\frac{d \alpha_{0}}{d+1}, 2 N(d+1), \frac{1}{d+1}\right]}
$$

This is useful, since [62,Eq. (7.13)] shows for $M \geq d+1$ that

$$
\Xi_{i, j}^{[k, M, \tau]} \leq C^{\prime} \cdot(1+|j-i|)^{|k|+\tau(d+1-M)} \quad \forall i, j \in \mathbb{Z}^{d} \backslash\{0\},
$$

where $C^{\prime}=C^{\prime}(\alpha, d, M, r, \tau,|k|)$.

Now, using that $w_{j}^{(s, \alpha)}=|j|^{s /(1-\alpha)}$ and $A_{j}=|j|^{\alpha_{0}}$ id, a straightforward computation shows that the quantity $L_{i, j}$ introduced in Lemma 7.4 satisfies

$$
L_{i, j}=\left\{\begin{array}{ll}
(|j| /|i|)^{2(d+1) \alpha_{0}+\frac{|s|}{1-\alpha}} & \text { if }|i| \leq|j|, \\
(|j| /|i|)^{-3(d+1) \alpha_{0}-\frac{|s|}{1-\alpha}} & \text { if }|i|>|j|
\end{array} \leq \max \left\{(|j| /|i|)^{\sigma},(|j| /|i|)^{-\sigma}\right\}\right.
$$


where we introduced $\sigma:=\frac{3 \alpha(d+1)+s_{0}}{1-\alpha} \in[0, \infty)$. In combination with Equations (7.5) and (7.6), we thus see that the matrix elements $\widehat{Y}_{i, j}$ introduced in Lemma 7.4 satisfy

$$
\begin{aligned}
0 & \leq \widehat{Y}_{i, j}=L_{i, j} Z_{i, j} \leq C \cdot \max \left\{(|j| /|i|)^{\sigma},(|j| /|i|)^{-\sigma}\right\} \cdot \Xi_{i, j}^{\left[d \alpha_{0}, N-2 d-2,1\right]} \\
& =C \cdot \max \left\{\Xi_{i, j}^{\left[\sigma+d \alpha_{0}, N-2 d-2,1\right]}, \Xi_{i, j}^{\left[d \alpha_{0}-\sigma, N-2 d-2,1\right]}\right\} \\
& \leq C \cdot C_{1} \cdot(1+|j-i|)^{\sigma+d \alpha_{0}+d+1-(N-2 d-2)} \\
& =C \cdot C_{1} \cdot(1+|j-i|)^{\sigma+d \alpha_{0}+3(d+1)-N}
\end{aligned}
$$

where $C_{1}=C_{1}\left(d, \alpha, N, r, s_{0}\right)$. From this, it is easy to see that $\|\widehat{Y}\|_{\text {Schur }} \leq C \cdot C_{2}<\infty$, provided that $N>4 d+3+\sigma+d \alpha_{0}=4 d+3+\tau$, where $C_{2}=C_{2}\left(d, \alpha, N, r, s_{0}\right)$. We have thus verified condition (iii) of Theorem 7.5.

Next, we show that $M_{0}<\infty$ for $M_{0}$ as defined in Proposition 7.1. The same arguments as for estimating $\widehat{Y}_{i, j}$ give

$$
\begin{aligned}
V_{i, j} & :=\max \left\{1,\left\|A_{j}^{-1} A_{i}\right\|^{d+1}\right\} W_{i, j} \\
& \leq C^{2} \max \left\{\Xi_{i, j}^{\left[\frac{d \alpha_{0}}{d+1}, 2 N(d+1), \frac{1}{d+1}\right]}, \Xi_{i, j}^{\left[\alpha_{0}\left(\frac{d}{d+1}-(d+1)\right), 2 N(d+1), 1 /(d+1)\right]}\right\} \\
& \leq C^{2} \cdot C_{3} \cdot(1+|j-i|)^{\alpha_{0} \frac{d^{2}+d+1}{d+1}+\frac{1}{d+1}(d+1-2 N(d+1))} \\
& \leq C^{2} \cdot C_{3} \cdot(1+|j-i|)^{1+\alpha_{0} \cdot(d+1)-2 N}
\end{aligned}
$$

where $C_{3}=C_{3}(\alpha, d, N, r)$. From this, we see that the constant $M_{0}$ introduced in Proposition 7.1 satisfies $M_{0}=\|V\|_{\text {Schur }} \leq C^{2} C_{4}<\infty$ for a constant $C_{4}=$ $C_{4}(\alpha, d, N, r)$, as soon as $N>\frac{1+d}{2}\left(1+\alpha_{0}\right)$, which is implied by $N>4 d+3+\sigma+d \alpha_{0}$. Thus, condition (iv) of Theorem 7.5 is satisfied.

Lastly, we verify condition (ii) of Theorem 7.5, that is, $\sum_{j \in \mathbb{Z}^{d} \backslash\{0\}}\left|\widehat{g}\left(S_{j}^{-1} \xi\right)\right|^{2} \geq A^{\prime}$ for all $\xi \in \widehat{\mathbb{R}}^{d}$, where $A^{\prime}:=c^{2}$, with $c>0$ as in Eq. (7.4). To see this, note that Eq. (7.4) implies $|\widehat{g}|^{2} \geq c^{2} \mathbb{1}_{Q}$, where we recall $Q=B_{r}(0)$. Hence, $\left|\widehat{g}\left(S_{j}^{-1} \xi\right)\right|^{2} \geq c^{2} \mathbb{1}_{Q_{j}}$, since $Q_{j}=S_{j} Q$. Finally, since $\mathcal{Q}^{(\alpha)}=\left(Q_{j}\right)_{j \in \mathbb{Z}^{d} \backslash\{0\}}$ is a cover of $\widehat{\mathbb{R}}^{d}$, we see $\sum_{j \in \mathbb{Z}^{d} \backslash\{0\}}\left|\widehat{g}\left(S_{j}^{-1} \xi\right)\right|^{2} \geq c^{2}=A^{\prime}$, as claimed.

Acknowledgements J. L. R. gratefully acknowledges support from the Austrian Science Fund (FWF): Y 1199 and P 29462-N35, and from the WWTF grant INSIGHT (MA16-053). J. v. V. acknowledges support from the Austrian Science Fund (FWF): P 29462-N35. J. v. V. is grateful for the hospitality and support of the Katholische Universität Eichstätt-Ingolstadt during his visit.

Open Access This article is licensed under a Creative Commons Attribution 4.0 International License, which permits use, sharing, adaptation, distribution and reproduction in any medium or format, as long as you give appropriate credit to the original author(s) and the source, provide a link to the Creative Commons licence, and indicate if changes were made. The images or other third party material in this article are included in the article's Creative Commons licence, unless indicated otherwise in a credit line to the material. If material is not included in the article's Creative Commons licence and your intended use is not permitted by statutory regulation or exceeds the permitted use, you will need to obtain permission directly from the copyright holder. To view a copy of this licence, visit http://creativecommons.org/licenses/by/4.0/. 


\section{Appendix A: Estimation of the $\mathcal{F} L^{1}$ norm}

\section{A.1: Sobolev Embeddings}

In this appendix we give an explicit bound for the constant implied in the estimate $\left\|\mathcal{F}^{-1} f\right\|_{L^{1}} \lesssim \max _{|\alpha| \leq d+1}\left\|\partial^{\alpha} f\right\|_{L^{1}}$. Similar, but more qualitative results in the noncommutative context can be found in $[36,51]$.

Lemma A.1 Let $d \in \mathbb{N}$ and $\alpha, c>0$. Define $g: \mathbb{R}^{d} \rightarrow(0, \infty), x \mapsto$ $\left(\max \left\{c,\|x\|_{\ell^{\infty}}\right\}\right)^{-\alpha}$. Then $\int_{\mathbb{R}^{d}} g(x) d x<\infty$ if and only if $\alpha>d$, and in this case

$$
\int_{\mathbb{R}^{d}} g(x) d x=\frac{2^{d}}{1-\frac{d}{\alpha}} \cdot c^{d-\alpha}
$$

Proof Let $\mu$ denote the Lebesgue measure on $\mathbb{R}^{d}$. We will use [25,Proposition 6.24], which shows for measurable $f: \mathbb{R}^{d} \rightarrow \mathbb{C}$ that

$$
\int_{\mathbb{R}^{d}}|f| d \mu=\int_{0}^{\infty} \lambda_{f}(\beta) d \beta
$$

where $\lambda_{f}(\beta):=\mu\left(\left\{x \in \mathbb{R}^{d}:|f(x)|>\beta\right\}\right)$. To compute the distribution function $\lambda_{g}$, first note that $g(x) \leq c^{-\alpha}$ for all $x \in \mathbb{R}^{d}$, and thus $\lambda_{g}(\beta)=0$ for $\beta \geq c^{-\alpha}$. For $0<\beta<c^{-\alpha}$, note that $g(x)>\beta$ is equivalent to $\|x\|_{\ell^{\infty}}<\beta^{-1 / \alpha}$, whence to $x \in$ $B_{\beta^{-1 / \alpha}}^{\| \cdot \ell^{\ell^{\infty}}}(0)$. Therefore, for any $\beta \in\left(0, c^{-\alpha}\right)$, we compute $\lambda_{g}(\beta)=\mu\left(B_{\beta^{-1 / \alpha}}^{\|\cdot\|_{\ell}}(0)\right)=$ $\left(2 \cdot \beta^{-1 / \alpha}\right)^{d}$, and thus

$$
\int_{\mathbb{R}^{d}} g(x) d x=\int_{0}^{\infty} \lambda_{g}(\beta) d \beta=2^{d} \cdot \int_{0}^{c^{-\alpha}} \beta^{-d / \alpha} d \beta
$$

which is finite if and only if $d / \alpha<1$. In the latter case, a direct calculation shows that

$$
\int_{\mathbb{R}^{d}} g(x) d x=\left.2^{d} \cdot \frac{\beta^{1-\alpha^{-1} d}}{1-\frac{d}{\alpha}}\right|_{\beta=0} ^{c^{-\alpha}}=\frac{2^{d}}{1-\frac{d}{\alpha}} \cdot\left(c^{-\alpha}\right)^{1-\alpha^{-1} d}=\frac{2^{d}}{1-\frac{d}{\alpha}} \cdot c^{d-\alpha}
$$

yielding the desired result.

The following result provides the announced estimate. For this, we use the usual Sobolev space

$$
W^{k, 1}\left(\mathbb{R}^{d}\right):=\left\{f \in L^{p}\left(\mathbb{R}^{d}\right): \partial^{\alpha} f \in L^{p}\left(\mathbb{R}^{d}\right) \forall \alpha \in \mathbb{N}_{0}^{d} \text { with }|\alpha| \leq k\right\}
$$

with norm $\|f\|_{W^{k, 1}}:=\sum_{|\alpha| \leq k}\left\|\partial^{\alpha} f\right\|_{L^{1}}$. 
Lemma A.2 Suppose $f \in W^{d+1,1}\left(\widehat{\mathbb{R}}^{d}\right)$. Then $\mathcal{F}^{-1} f \in L^{1}\left(\mathbb{R}^{d}\right)$ with

$$
\left\|\mathcal{F}^{-1} f\right\|_{L^{1}} \leq \frac{d+1}{\pi^{d}} \cdot \max _{\theta \in \mathbb{I}}\left\|\partial^{\theta} f\right\|_{L^{1}},
$$

where $\mathbb{I}:=\{0\} \cup\left\{(d+1) e_{\ell}: \ell \in \underline{d}\right\} \subset \mathbb{N}_{0}^{d}$, with $\left(e_{k}\right)_{k=1}^{d}$ denoting the standard basis of $\mathbb{R}^{d}$.

Proof Since $\mathcal{S}\left(\widehat{\mathbb{R}}^{d}\right) \subset W^{d+1,1}\left(\widehat{\mathbb{R}}^{d}\right)$ is dense (see e.g. [3,E10.8]), and since $\mathcal{F}^{-1} f_{n} \rightarrow \mathcal{F}^{-1} f$ uniformly if $f_{n} \rightarrow f$ in $W^{d+1,1}\left(\widehat{\mathbb{R}}^{d}\right) \hookrightarrow L^{1}\left(\widehat{\mathbb{R}}^{d}\right)$, it suffices-in view of Fatou's lemma-to prove the estimate for $f \in \mathcal{S}\left(\widehat{\mathbb{R}}^{d}\right)$. In this case, elementary properties of the Fourier transform yield for all $\alpha \in \mathbb{N}_{0}^{d}$ and $x \in \mathbb{R}^{d}$ the estimate

$$
\left|x^{\alpha} \cdot \mathcal{F}^{-1} f(x)\right|=(2 \pi)^{-|\alpha|} \cdot\left|\mathcal{F}^{-1}\left(\partial^{\alpha} f\right)(x)\right| \leq(2 \pi)^{-|\alpha|} \cdot\left\|\partial^{\alpha} f\right\|_{L^{1}} .
$$

Next, using the auxiliary function $g: \mathbb{R}^{d} \rightarrow(0, \infty), \quad x \mapsto\left(\max \left\{(2 \pi)^{-1}\right.\right.$, $\left.\left.\|x\|_{\ell^{\infty}}\right\}\right)^{-(d+1)}$, it follows that

$$
\begin{aligned}
\left|\mathcal{F}^{-1} f(x)\right| & =g(x) \cdot \max \left\{(2 \pi)^{-(d+1)},\|x\|_{\ell^{\infty}}^{d+1}\right\} \cdot\left|\mathcal{F}^{-1} f(x)\right| \\
& =g(x) \cdot \max \left\{(2 \pi)^{-(d+1)} \cdot\left|\mathcal{F}^{-1} f(x)\right|, \quad \max _{\ell \in \underline{d}}\left|x_{\ell}^{d+1} \cdot \mathcal{F}^{-1} f(x)\right|\right\} \\
& \leq g(x) \cdot \max \left\{(2 \pi)^{-(d+1)} \cdot\|f\|_{L^{1}}, \quad \max _{\ell \in \underline{d}}\left[(2 \pi)^{-(d+1)} \cdot\left\|\partial_{\ell}^{d+1} f\right\|_{L^{1}}\right]\right\} \\
& \leq g(x) \cdot(2 \pi)^{-(d+1)} \cdot \max _{\theta \in \mathbb{I}}\left\|\partial^{\theta} f\right\|_{L^{1}} .
\end{aligned}
$$

Hence, it remains to compute the integral $\int_{\mathbb{R}^{d}} g(x) d x$. For this, note that an application of Lemma A.1 (with $c=(2 \pi)^{-1}$ and $\alpha=d+1$ ) gives $\int g(x) d x=\frac{2^{d}}{1-\alpha^{-1} d} \cdot c^{d-\alpha}=$ $2^{d+1} \pi \cdot(d+1)$, and thus

$$
\left\|\mathcal{F}^{-1} f\right\|_{L^{1}} \leq 2^{d+1} \pi \cdot(d+1) \cdot(2 \pi)^{-(d+1)} \cdot \max _{\theta \in \mathbb{I}}\left\|\partial^{\theta} f\right\|_{L^{1}}=\frac{d+1}{\pi^{d}} \cdot \max _{\theta \in \mathbb{I}}\left\|\partial^{\theta} f\right\|_{L^{1}}
$$

which completes the proof.

\section{A.2: The Chain Rule}

Lemma A. 2 allows to estimate the $\mathcal{F} L^{1}$ norm of $f$ in terms of the $L^{1}$ norms of certain derivatives of $f$. In many cases, we will have $f=g \circ A$, where we have good control over the derivatives of $g$. In such cases, the following lemma will be helpful.

Lemma A.3 ([60,Lemma 2.6])

Let $d, k \in \mathbb{N}, A \in \mathbb{R}^{d \times d}$, and $f \in C^{k}\left(\mathbb{R}^{d}\right)$ be arbitrary. Let $\left(e_{1}, \ldots, e_{d}\right)$ denote the standard basis of $\mathbb{R}^{d}$, let $i_{1}, \ldots, i_{k} \in \underline{d}$, and define $\alpha:=\sum_{m=1}^{k} e_{i_{m}} \in \mathbb{N}_{0}^{d}$. 
Then $|\alpha|=k$, and

$$
\partial^{\alpha}(f \circ A)(x)=\sum_{\ell_{1}, \ldots, \ell_{k} \in \underline{d}}\left[A_{\ell_{1}, i_{1}} \cdots A_{\ell_{k}, i_{k}} \cdot\left(\partial_{\ell_{1}} \cdots \partial_{\ell_{k}} f\right)(A x)\right] \quad \forall x \in \mathbb{R}^{d} .
$$

\section{A.3: The Norm of a Reciprocal}

Lemma A.4 Let $m \in \mathbb{N}$ and let $U \subset \mathbb{R}$ be open. Suppose that $f \in C^{m}(U)$ never vanishes on $U$. Let $A>0, K \geq 0$, and $x_{0} \in U$ be such that

$$
\left|f\left(x_{0}\right)\right| \geq A^{-1} \text { and }\left|f^{(\ell)}\left(x_{0}\right)\right| \leq K \quad \forall 1 \leq \ell \leq m
$$

Then the reciprocal $F:=1 / f$ of $f$ satisfies

$$
\left|\frac{d^{\ell}}{d x^{\ell}}\right|_{x=x_{0}} F(x) \mid \leq C_{m} \cdot A \cdot \max \left\{A K,(A K)^{\ell}\right\}
$$

for all $1 \leq \ell \leq m$, where the constant $C_{m}$ satisfies, for all $1 \leq \ell \leq m$,

$$
1 \leq C_{m} \leq 3 \sqrt{m} \cdot\left(\frac{\frac{0.8}{e} \cdot m^{2}}{\ln (1+m)}\right)^{m}
$$

Proof Setting $g: \mathbb{R} \backslash\{0\} \rightarrow \mathbb{R}, t \mapsto t^{-1}$, we have $F=g \circ f$. Therefore, the "set partition version" of Faa di Bruno's formula, see for instance [42,p. 219], shows for $1 \leq \ell \leq m$ that

$$
F^{(\ell)}\left(x_{0}\right)=\sum_{\pi \in P_{\ell}}\left[g^{(|\pi|)}\left(f\left(x_{0}\right)\right) \prod_{B \in \pi} f^{(|B|)}\left(x_{0}\right)\right],
$$

where $P_{\ell} \subset 2^{2^{\underline{\ell}}}$ denotes the sets of all partitions of the set $\underline{\ell}:=\{1, \ldots, \ell\}$. Phrased differently, the set $P_{\ell}$ contains exactly those subsets $\pi \subset 2^{\underline{\ell}}$ of the power set $2^{\underline{\ell}}$ for which $\underline{\ell}=\biguplus \pi$ and $B \neq \emptyset$ for all $B \in \pi$. For each $\pi \in P_{\ell}$, we denote by $|\pi|$ the number of blocks of the partition determined by $\pi$; that is, $|\pi|$ is the number of elements of $\pi$. Likewise, for a block $B \in \pi$, we denote by $|B|$ the size of the block, that is, the number of elements of $B$.

An induction argument shows that $g^{(k)}(t)=(-1)^{k} \cdot k ! \cdot t^{-(k+1)}$ for all $k \in \mathbb{N}_{0}$. Therefore, for arbitrary $\pi \in P_{\ell}$, it follows that $\left|g^{(|\pi|)}\left(f\left(x_{0}\right)\right)\right|=|\pi| ! \cdot\left|f\left(x_{0}\right)\right|^{-(1+|\pi|)} \leq$ $\ell ! \cdot A^{1+|\pi|}$, since any $\pi \in P_{\ell}$ satisfies $\ell=\sum_{B \in \pi}|B| \geq \sum_{B \in \pi} 1=|\pi|$. Similarly, it follows that

$$
\prod_{B \in \pi}\left|f^{(|B|)}\left(x_{0}\right)\right| \leq \prod_{B \in \pi} K=K^{|\pi|}
$$


for all $\pi \in P_{\ell}$. Combining these observations shows that

$$
\left|F^{(\ell)}\left(x_{0}\right)\right| \leq \sum_{\pi \in P_{\ell}}\left(\ell ! \cdot A \cdot(A K)^{|\pi|}\right) \leq A \cdot \max \left\{A K,(A K)^{\ell}\right\} \cdot \ell ! \cdot\left|P_{\ell}\right|
$$

where we used again that $1 \leq|\pi| \leq \ell$ for $\pi \in P_{\ell}$. Since $\ell ! \leq m$ ! and $\left|P_{\ell}\right| \leq\left|P_{m}\right|$ for $\ell \leq m$, it suffices to show that $C_{m}:=m ! \cdot\left|P_{m}\right|$ satisfies the bound stated in the lemma. Here, the cardinalities $\left|P_{m}\right|$ are the so-called Bell numbers. For these, [9,Theorem 2.1] provides the bound $\left|P_{m}\right| \leq\left(\frac{0.8 \cdot m}{\ln (1+m)}\right)^{m}$. Furthermore, the version of Stirling's formula derived in [50] shows that

$$
m ! \leq \sqrt{2 \pi} \cdot e^{1 / 12} \cdot(m / e)^{m} \cdot \sqrt{m} \leq 3 \cdot(m / e)^{m} \cdot \sqrt{m}
$$

Combining these estimates gives the desired result.

\section{Appendix B: Proof of Proposition 3.13}

(i) Let $f \in \mathcal{S}_{\mathcal{O}}\left(\mathbb{R}^{d}\right)$ and set $K:=\operatorname{supp} \widehat{f} \subset \mathcal{O}$. For $i \in I$, the set $U_{i}:=\varphi_{i}^{-1}(\mathbb{C} \backslash\{0\})$ is open. Moreover, since $\sum_{i \in I} \varphi_{i} \equiv 1$ on $\mathcal{O}$, it follows that $\mathcal{O}=\bigcup_{i \in I} U_{i}$. By compactness of $K$, there exists a finite subset $I_{K} \subset I$ satisfying $K \subset \bigcup_{\ell \in I_{K}} U_{\ell} \subset$ $\bigcup_{\ell \in I_{K}} Q_{\ell}$. Therefore, for any $i \in I$ satisfying $Q_{i} \cap K \neq \emptyset$, necessarily $\emptyset \neq Q_{i} \cap K \subset$ $Q_{i} \cap \bigcup_{\ell \in I_{K}} Q_{\ell}$, and hence $i \in I_{K}^{*}:=\bigcup_{\ell \in I_{K}} \ell^{*}$, which is a finite subset of $I$. By contraposition, we have $Q_{i} \cap K=\emptyset$, and hence $\varphi_{i} \cdot \widehat{f} \equiv 0$, for all $i \in I \backslash I_{K}^{*}$.

Next, for each $i \in I_{K}^{*}$, clearly $\varphi_{i} \cdot \widehat{f} \in C_{c}^{\infty}(\mathcal{O})$, and thus $\left\|\mathcal{F}^{-1}\left(\varphi_{i} \cdot \widehat{f}\right)\right\|_{L^{p}}<\infty$. Therefore, setting $M:=\max _{i \in I_{K}^{*}}\left\|\mathcal{F}^{-1}\left(\varphi_{i} \cdot \widehat{f}\right)\right\|_{L^{p}}<\infty$ gives

$$
\|f\|_{\mathcal{D}\left(\mathcal{Q}, L^{p}, \ell_{w}^{q}\right)}=\left\|\left(\left\|\mathcal{F}^{-1}\left(\varphi_{i} \cdot \widehat{f}\right)\right\|_{L^{p}}\right)_{i \in I}\right\|_{\ell_{w}^{q}} \leq\left\|(M)_{i \in I_{K}^{*}}\right\|_{\ell_{w}^{q}}<\infty
$$

which shows that $f \in \mathcal{D}\left(\mathcal{Q}, L^{p}, \ell_{w}^{q}\right)$.

(ii) Let $p, q \in[1, \infty)$. Recall the notation $C_{\Phi}=\sup _{i \in I}\left\|\mathcal{F}^{-1} \varphi_{i}\right\|_{L^{1}}$ from Definition 3.3. Let $f \in \mathcal{D}\left(\mathcal{Q}, L^{p}, \ell_{w}^{q}\right)$ and $\varepsilon>0$ be arbitrary. Note $c=\left(c_{i}\right)_{i \in I} \in \ell_{w}^{q}(I)$, where $c_{i}:=\left\|\mathcal{F}^{-1}\left(\varphi_{i} \cdot \widehat{f}\right)\right\|_{L^{p}}$ for $i \in I$. Since $\|c\|_{\ell_{w}^{q}}=\|f\|_{\mathcal{D}\left(\mathcal{Q}, L^{p}, \ell_{w}^{q}\right)}<\infty$ and since $q<\infty$, there exists a finite set $I_{0}=I_{0}(\varepsilon, f) \subset I$ such that the sequence $\tilde{c}:=c \cdot \mathbb{1}_{I \backslash I_{0}}$ satisfies

$$
\|\widetilde{c}\|_{\ell_{w}^{q}}<\left(C_{\Phi} \cdot\left\|\Gamma_{\mathcal{Q}}\right\|_{\ell_{w}^{q} \rightarrow \ell_{w}^{q}}\right)^{-2} \cdot \frac{\varepsilon}{2}
$$

For each $i \in I_{0}^{*}:=\bigcup_{\ell \in I_{0}} \ell^{*}$, let $c_{i}^{*}:=\left(\Gamma_{\mathcal{Q}} c\right)_{i}=\sum_{\ell \in i^{*}} c_{\ell}$ and choose some $h_{i} \in \mathcal{S}\left(\mathbb{R}^{d}\right)$ such that $\left\|\mathcal{F}^{-1}\left(\varphi_{i}^{*} \cdot \widehat{f}\right)-h_{i}\right\|_{L^{p}} \leq \delta \cdot c_{i}^{*}$, where $\delta:=(\varepsilon / 2) \cdot\left(C_{\Phi} \cdot\right.$ $\left.\left\|\Gamma_{\mathcal{Q}}\right\|_{\ell_{w}^{q} \rightarrow \ell_{w}^{q}}\right)^{-2} \cdot\left(1+\|c\|_{\ell_{w}^{q}}\right)^{-1}$. This is possible since we have $p<\infty$, and since if $c_{i}^{*}=0$, then $\left\|\mathcal{F}^{-1}\left(\varphi_{i}^{*} \cdot \widehat{f}\right)\right\|_{L^{p}} \leq \sum_{\ell \in i^{*}}\left\|\mathcal{F}^{-1}\left(\varphi_{\ell} \widehat{f}\right)\right\|_{L^{p}}=c_{i}^{*}=0$. 
Define $g_{i}:=\widehat{h_{i}} \in \mathcal{S}\left(\widehat{\mathbb{R}}^{d}\right)$ for $i \in I_{0}^{*}$, and $g_{i}:=0$ for $i \in I \backslash I_{0}^{*}$. We claim that

$$
\left\|\mathcal{F}^{-1}\left(\varphi_{i}^{*} \cdot \widehat{f}\right)-\mathcal{F}^{-1} g_{i}\right\|_{L^{p}} \leq\left(\Gamma_{\mathcal{Q}} \tilde{c}+\delta \cdot \Gamma_{\mathcal{Q}} c\right)_{i}
$$

for all $i \in I$. To show this, distinguish the two cases $i \in I_{0}^{*}$ and $i \in I \backslash I_{0}^{*}$. In the first case,

$$
\left\|\mathcal{F}^{-1}\left(\varphi_{i}^{*} \cdot \widehat{f}\right)-\mathcal{F}^{-1} g_{i}\right\|_{L^{p}}=\left\|\mathcal{F}^{-1}\left(\varphi_{i}^{*} \cdot \widehat{f}\right)-h_{i}\right\|_{L^{p}} \leq \delta \cdot c_{i}^{*}=\delta \cdot\left(\Gamma_{\mathcal{Q}} c\right)_{i}
$$

by choice of $h_{i}$. Since, furthermore, $\left(\Gamma_{\mathcal{Q}} \widetilde{c}\right)_{i} \geq 0$, the estimate (B.1) holds in the first case. For the second case, we have $g_{i}=0$. Furthermore, $i \notin I_{0}^{*}$ and thus $\ell \notin I_{0}$ for all $\ell \in i^{*}$. Therefore,

$$
\begin{aligned}
& \left\|\mathcal{F}^{-1}\left(\varphi_{i}^{*} \cdot \widehat{f}\right)-\mathcal{F}^{-1} g_{i}\right\|_{L^{p}}=\left\|\mathcal{F}^{-1}\left(\varphi_{i}^{*} \cdot \widehat{f}\right)\right\|_{L^{p}} \\
& \leq \sum_{\ell \in i^{*}}\left[\mathbb{1}_{I \backslash I_{0}}(\ell) \cdot\left\|\mathcal{F}^{-1}\left(\varphi_{\ell} \cdot \widehat{f}\right)\right\|_{L^{p}}\right]=\left(\Gamma_{\mathcal{Q}} \widetilde{c}\right)_{i} .
\end{aligned}
$$

As in the first case, we thus see that estimate (B.1) holds.

Define $g:=\mathcal{F}^{-1}\left(\sum_{i \in I} \varphi_{i} \cdot g_{i}\right)$. Then $g \in \mathcal{S}_{\mathcal{O}}\left(\mathbb{R}^{d}\right)$ since $g_{i}=0$ for all but finitely many $i \in I$. Next, note that $\varphi_{i} \varphi_{i}^{*}=\varphi_{i}$, and hence

$$
\varphi_{\ell} \cdot \widehat{f-g}=\varphi_{\ell} \cdot\left(\sum_{i \in I} \varphi_{i} \cdot \widehat{f}-\sum_{i \in I} \varphi_{i} \cdot g_{i}\right)=\varphi_{\ell} \cdot \sum_{i \in \ell^{*}}\left[\varphi_{i} \cdot\left(\varphi_{i}^{*} \widehat{f}-g_{i}\right)\right] .
$$

Using Young's inequality, we thus get

$$
\begin{aligned}
\left\|\mathcal{F}^{-1}\left(\varphi_{\ell} \cdot \widehat{f-g}\right)\right\|_{L^{p}} & \leq\left\|\mathcal{F}^{-1} \varphi_{\ell}\right\|_{L^{1}} \cdot \sum_{i \in \ell^{*}}\left(\left\|\mathcal{F}^{-1} \varphi_{i}\right\|_{L^{1}} \cdot\left\|\mathcal{F}^{-1}\left(\varphi_{i}^{*} \widehat{f}\right)-\mathcal{F}^{-1} g_{i}\right\|_{L^{p}}\right) \\
& \leq C_{\Phi}^{2} \cdot \sum_{i \in \ell^{*}}\left(\Gamma_{\mathcal{Q}} \tilde{c}+\delta \cdot \Gamma_{\mathcal{Q}} c\right)_{i}=C_{\Phi}^{2} \cdot\left[\Gamma_{\mathcal{Q}}\left(\Gamma_{\mathcal{Q}} \tilde{c}+\delta \cdot \Gamma_{\mathcal{Q}} c\right)\right]_{\ell}
\end{aligned}
$$

where the last inequality follows by (B.1). This finally implies

$$
\begin{aligned}
& \|f-g\|_{\mathcal{D}\left(\mathcal{Q}, L^{p}, \ell_{w}^{q}\right)} \leq C_{\Phi}^{2} \cdot\left\|\Gamma_{\mathcal{Q}}\right\| \cdot\left(\left\|\Gamma_{\mathcal{Q}} \widetilde{c}\right\|_{\ell_{w}^{q}}\right. \\
& \left.\quad+\delta \cdot\left\|\Gamma_{\mathcal{Q}} c\right\|_{\ell_{w}^{q}}\right) \leq\left(C_{\Phi}\left\|\Gamma_{\mathcal{Q}}\right\|\right)^{2} \cdot\left(\|\widetilde{c}\|_{\ell_{w}^{q}}+\delta \cdot\|c\|_{\ell_{w}^{q}}\right) \leq \varepsilon,
\end{aligned}
$$

which completes the proof of (ii).

(iii) Since $\mathcal{Q}$ is a decomposition cover, the index set $I$ is countably infinite. Indeed, the sets $\left(\varphi_{i}^{-1}(\mathbb{C} \backslash\{0\})\right)_{i \in I}$ form an open cover of $\mathcal{O}$. Since $\mathcal{O}$ is second countable, there is a countable $I_{0} \subset I$ such that $\mathcal{O} \subset \bigcup_{i \in I_{0}} \varphi_{i}^{-1}(\mathbb{C} \backslash\{0\}) \subset \bigcup_{i \in I_{0}} Q_{i}$. Finally, for $i \in I$, we have $\emptyset \neq Q_{i} \subset \mathcal{O} \subset \bigcup_{\ell \in I_{0}} Q_{\ell}$, and hence $i \in \ell^{*}$ for some $\ell \in I_{0}$. In other words, $I \subset \bigcup_{\ell \in I_{0}} \ell^{*}$ is countable as a countable union of finite sets. Finally, if $I$ was finite, then $\sum_{i \in I} \varphi_{i} \in C_{c}(\mathcal{O})$, in contradiction to $\mathcal{O}$ being open and to $\sum_{i \in I} \varphi_{i} \equiv 1$ on $\mathcal{O}$. Thus, we can write $I=\left\{i_{n}: n \in \mathbb{N}\right\}$ for pairwise distinct $\left(i_{n}\right)_{n \in \mathbb{N}}$. 
For each $i \in I$, we have $f_{i}:=\mathcal{F}^{-1}\left(\varphi_{i} \widehat{f}\right) \in L^{p}\left(\mathbb{R}^{d}\right)$ with supp $\widehat{f}_{i} \subset \operatorname{supp} \varphi_{i} \subset U_{i}$ for the open set $U_{i}:=\left(\varphi_{i}^{*}\right)^{-1}(\mathbb{C} \backslash\{0\}) \subset Q_{i}^{*} \subset \mathcal{O}$, since $\varphi_{i}^{*} \varphi_{i}=\varphi_{i}$. Now, for each fixed $i \in I$, [61,Lemma 3.2] yields a sequence $\left(f_{i}^{(n)}\right)_{n \in \mathbb{N}}$ of Schwartz functions such that $\left|f_{i}^{(n)}\right| \leq\left|f_{i}\right|$ and $f_{i}^{(n)} \underset{n \rightarrow \infty}{\longrightarrow} f_{i}$ pointwise, and such that supp $\widehat{f_{i}^{(n)}} \subset$ $B_{1 / n}\left(\operatorname{supp} \varphi_{i}\right)$, where $B_{1 / n}\left(\operatorname{supp} \varphi_{i}\right):=\left\{\xi \in \widehat{\mathbb{R}}^{d}: \operatorname{dist}\left(\xi, \operatorname{supp} \varphi_{i}\right) \leq n^{-1}\right\}$. By choos$\operatorname{ing} N_{i} \in \mathbb{N}$ with $B_{1 / N_{i}}\left(\operatorname{supp} \varphi_{i}\right) \subset U_{i}$, and by replacing $f_{i}^{(1)}, \ldots, f_{i}^{\left(N_{i}\right)}$ by $f_{i}^{\left(N_{i}\right)}$, we get supp $\widehat{f_{i}^{(n)}} \subset U_{i} \subset Q_{i}^{*} \subset \mathcal{O}$ for all $i \in I$ and $n \in \mathbb{N}$.

Note that we have $f_{i}^{(n)} \underset{n \rightarrow \infty}{\stackrel{\mathcal{S}^{\prime}\left(\mathbb{R}^{d}\right)}{\longrightarrow}} f_{i}$. Indeed, if $p<\infty$, then this follows from $f_{i}^{(n)} \underset{n \rightarrow \infty}{\stackrel{L^{p}}{\longrightarrow}} f_{i}$, which is a consequence of the dominated convergence theorem since $\left|f_{i}^{(n)}\right| \leq\left|f_{i}\right| \in L^{p}$ and $f_{i}^{(n)} \rightarrow f_{i}$ pointwise. If $p=\infty$ and $h \in \mathcal{S}\left(\mathbb{R}^{d}\right)$, then $f_{i}^{(n)} \cdot h \rightarrow f_{i} \cdot h$ pointwise, and we have the estimate $\left|f_{i}^{(n)} \cdot h\right| \leq\left|f_{i} \cdot h\right| \leq\left\|f_{i}\right\|_{L^{\infty}} \cdot|h| \in$ $L^{1}$, whence $\left\langle f_{i}^{(n)}, h\right\rangle_{\mathcal{S}^{\prime}, \mathcal{S}} \rightarrow\left\langle f_{i}, h\right\rangle_{\mathcal{S}^{\prime}, \mathcal{S}}$ by dominated convergence.

Now, define $g_{N}:=\sum_{n=1}^{N} f_{i_{n}}^{(N)} \in \mathcal{S}_{\mathcal{O}}\left(\mathbb{R}^{d}\right)$. We first verify that $g_{N} \rightarrow f$ with convergence in $Z^{\prime}(\mathcal{O})$. To see this, let $\psi \in Z(\mathcal{O})$ be arbitrary. Then $\mathcal{F}^{-1} \psi \in C_{c}^{\infty}(\mathcal{O})$, so that $K:=\operatorname{supp} \mathcal{F}^{-1} \psi \subset \mathcal{O}$ is compact. Precisely as in the proof of Part (i), we thus see that there is a finite set $I_{K} \subset I$ such that $Q_{i} \cap K=\emptyset$ for all $i \in I \backslash I_{K}$. Therefore, $U_{i} \cap K \subset Q_{i}^{*} \cap K=\emptyset$, and hence $\widehat{f_{i}^{(n)}} \cdot \mathcal{F}^{-1} \psi \equiv 0$, for all $i \in I \backslash I_{K}^{*}$. Now, choose $N_{0}=N_{0}(K) \in \mathbb{N}$ such that $I_{K}^{*} \subset\left\{i_{1}, \ldots, i_{N_{0}}\right\}$. If $N \geq N_{0}$, we then have

$$
\begin{aligned}
\left\langle g_{N}, \psi\right\rangle_{Z^{\prime}, Z}=\left\langle\widehat{g_{N}}, \mathcal{F}^{-1} \psi\right\rangle_{\mathcal{D}^{\prime}(\mathcal{O}), \mathcal{D}} & \left.=\sum_{n=1}^{N} \widehat{\left\langle f_{i_{n}}^{(N)}\right.}, \mathcal{F}^{-1} \psi\right\rangle_{\mathcal{D}^{\prime}(\mathcal{O}), \mathcal{D}} \\
& \left.=\sum_{i \in I_{K}^{*}} \widehat{\left\langle f_{i}^{(N)}\right.}, \mathcal{F}^{-1} \psi\right\rangle_{\mathcal{D}^{\prime}(\mathcal{O}), \mathcal{D}},
\end{aligned}
$$

where the last equality follows since $\left\{i_{1}, \ldots, i_{N}\right\} \supset I_{K}^{*}$ and $\widehat{f_{i}^{(N)}} \cdot \mathcal{F}^{-1} \psi \equiv 0$ for $i \in$ $I \backslash I_{K}^{*}$. Next, using that $f_{i}^{(N)} \rightarrow f_{i}$ in $\mathcal{S}^{\prime}$ and noting that $\mathcal{F}^{-1} \psi=\sum_{i \in I} \varphi_{i} \mathcal{F}^{-1} \psi=$ $\sum_{i \in I_{K}^{*}} \varphi_{i} \mathcal{F}^{-1} \psi$, we see that

$$
\begin{aligned}
\left\langle g_{N}, \psi\right\rangle_{Z^{\prime}, Z} \underset{N \rightarrow \infty}{\longrightarrow} & \sum_{i \in I_{K}^{*}}\left\langle\widehat{f_{i}}, \mathcal{F}^{-1} \psi\right\rangle_{\mathcal{D}^{\prime}(\mathcal{O}), \mathcal{D}} \\
& =\sum_{i \in I_{K}^{*}}\left\langle\varphi_{i} \widehat{f}, \mathcal{F}^{-1} \psi\right\rangle_{\mathcal{D}^{\prime}(\mathcal{O}), \mathcal{D}} \\
& =\left\langle\widehat{f}, \mathcal{F}^{-1} \psi\right\rangle_{\mathcal{D}^{\prime}(\mathcal{O}), \mathcal{D}} \\
& =\langle f, \psi\rangle_{Z^{\prime}, Z}
\end{aligned}
$$

Thus, $g_{N} \underset{N \rightarrow \infty}{\longrightarrow} f$ with convergence in $Z^{\prime}(\mathcal{O})$. 
Finally, we construct a sequence $F=\left(F_{i}\right)_{i \in I} \in \ell_{w}^{q}\left(I ; L^{p}\right)$ such that each $g_{N}$ is $(F, \Phi)$-dominated. To this end, set $F_{i}:=\sum_{\ell \in i^{* *}}\left|\breve{\varphi}_{i}\right| *\left|f_{\ell}\right|$, where $f_{\ell}:=\mathcal{F}^{-1}\left(\varphi_{\ell} \cdot \widehat{f}\right)$. Note because of supp $\widehat{f_{i_{n}}^{(N)}} \subset Q_{i_{n}}^{*}$ that $\varphi_{i} \cdot \widehat{f_{i_{n}}^{(N)}} \not \equiv 0$ can only hold for $i_{n} \in i^{* *}$. Therefore, since $\left|f_{i}^{(m)}\right| \leq\left|f_{i}\right|$, we get

$$
\begin{aligned}
\left|\mathcal{F}^{-1}\left(\varphi_{i} \widehat{g_{N}}\right)\right| & =\left|\mathcal{F}^{-1}\left(\varphi_{i} \cdot \sum_{n \in \underline{N}: i_{n} \in i^{* *}} \widehat{f_{i_{n}}^{(N)}}\right)\right| \leq \sum_{n \in \underline{N}: i_{n} \in i^{* *}}\left|\mathcal{F}^{-1}\left(\varphi_{i} \cdot \widehat{f_{i_{n}}^{(N)}}\right)\right| \\
& \leq \sum_{\ell \in i^{* *}}\left|\check{\varphi}_{i}\right| *\left|f_{\ell}\right|=F_{i} .
\end{aligned}
$$

Finally, setting $c=\left(c_{i}\right)_{i \in I}$ with $c_{i}:=\left\|\mathcal{F}^{-1}\left(\varphi_{i} \widehat{f}\right)\right\|_{L^{p}}$, we see because of $i^{* *}=$ $\bigcup_{j \in i^{*}} j^{*}$ that

$\left\|F_{i}\right\|_{L^{p}} \leq \sum_{\ell \in i^{* *}}\left\|\left|\mathcal{F}^{-1} \varphi_{i}\right| *\left|f_{\ell}\right|\right\|_{L^{p}} \leq \sum_{j \in i^{*}} \sum_{\ell \in j^{*}}\left\|\mathcal{F}^{-1} \varphi_{i}\right\|_{L^{1}} \cdot\left\|f_{\ell}\right\|_{L^{p}} \leq C_{\Phi} \cdot\left(\Gamma_{\mathcal{Q}} \Gamma_{\mathcal{Q}} c\right)_{i}$

Thus, $F \in \ell_{w}^{q}\left(I ; L^{p}\right)$ with $\|F\|_{\ell_{w}^{q}\left(I ; L^{p}\right)} \leq C_{\Phi}\left\|\Gamma_{\mathcal{Q}}\right\|_{\ell_{w}^{q} \rightarrow \ell_{w}^{q}}^{2} \cdot\|f\|_{\mathcal{D}\left(\mathcal{Q}, L^{p}, \ell_{w}^{q}\right)}$, since $\|f\|_{\mathcal{D}\left(\mathcal{Q}, L^{p}, \ell_{w}^{q}\right)}=\|c\|_{\ell_{w}^{q}}$.

\section{Appendix C: Proof of Proposition 5.7}

Before proving Proposition 5.7, we first collect a few properties of the "generalized multiplication operation" $\odot$ introduced in Definition 5.5.

Lemma C.1 Let $p \in[1, \infty]$. For $f, g \in \mathcal{F} L^{1}\left(\mathbb{R}^{d}\right)$ and $h \in \mathcal{F} L^{p}\left(\mathbb{R}^{d}\right)$, the following properties hold:

(i) $f \odot(g \odot h)=(f \odot g) \odot h$.

(ii) If $f \in \mathcal{S}\left(\widehat{\mathbb{R}}^{d}\right)$, then $f \odot h=f \cdot h$.

(iii) If $p \in[1,2]$, then $f \odot h=f \cdot h$.

(iv) We have $\operatorname{supp}(f \odot h) \subset \operatorname{supp} f \cap \operatorname{supp} h$, where the support is understood in the sense of tempered distributions.

Proof (i) Note that $\breve{f}, \breve{g} \in L^{1}\left(\mathbb{R}^{d}\right)$ and $\breve{h} \in L^{p}\left(\mathbb{R}^{d}\right)$. Thus, Young's inequality shows for almost all $x \in \mathbb{R}^{d}$ that $(|\breve{f}| *(|\breve{g}| *|\breve{h}|))(x)<\infty$. For each such $x$, a standard calculation using Fubini's theorem shows $((\breve{f} * \breve{g}) * \breve{h})(x)=(\breve{f} *(\breve{g} * \breve{h}))(x)$. Hence, both sides are identical as tempered distributions. Thus, $(f \odot g) \odot h=f \odot(g \odot h)$. (ii) This was already observed in Remark 5.6.

(iii) It is well-known that if $p \in[1,2]$, then $\widehat{\varphi * \psi}=\widehat{\varphi} \cdot \widehat{\psi}$ for $\varphi \in L^{1}\left(\mathbb{R}^{d}\right)$ and $\psi \in L^{p}\left(\mathbb{R}^{d}\right)$. Indeed, for $\varphi, \psi \in \mathcal{S}\left(\mathbb{R}^{d}\right)$, the identity is clear; furthermore, it follows from the Hausdorff-Young inequality that as elements of $L^{p^{\prime}}\left(\mathbb{R}^{d}\right)$, both sides of the identity depend continuously on $\varphi \in L^{1}\left(\mathbb{R}^{d}\right)$ and $\psi \in L^{p}\left(\mathbb{R}^{d}\right)$. Therefore, $f \odot h=$ $\mathcal{F}[\check{f} * \check{h}]=f \cdot h$. 
(iv) Let $\varphi \in C_{c}^{\infty}\left(\widehat{\mathbb{R}}^{d}\right)$ with $\operatorname{supp} \varphi \subset \widehat{\mathbb{R}}^{d} \backslash \operatorname{supp} f$. There is $\psi \in C_{c}^{\infty}\left(\widehat{\mathbb{R}}^{d}\right)$ with $\varphi=\varphi \cdot \psi$ and supp $\psi \subset \widehat{\mathbb{R}}^{d} \backslash \operatorname{supp} f$. Furthermore, by combining Properties (i) and (ii), we see that

$$
\psi \cdot(f \odot h)=\psi \odot(f \odot h)=(\psi \odot f) \odot h=(\psi \cdot f) \odot h=0 .
$$

Because of $\varphi=\psi \cdot \varphi$, this entails $\langle f \odot h, \varphi\rangle_{\mathcal{S}^{\prime}, \mathcal{S}}=\langle\psi \cdot(f \odot h), \varphi\rangle_{\mathcal{S}^{\prime}, \mathcal{S}}=0$. Since this holds for every $\varphi \in C_{c}^{\infty}\left(\widehat{\mathbb{R}}^{d}\right)$ with $\operatorname{supp} \varphi \subset \widehat{\mathbb{R}}^{d} \backslash \operatorname{supp} f$, we see $\operatorname{supp}(f \odot h) \subset$ $\operatorname{supp} f$. The argument for $\operatorname{supp}(f \odot h) \subset \operatorname{supp} h$ is similar.

With this preparation, we can now provide the proof of Proposition 5.7.

Proof of Proposition 5.7 Before proving the claims, we show that $\Phi_{h}$ is well-defined, with unconditional convergence in $Z^{\prime}(\mathcal{O})$ of the defining series. For brevity, let $\psi_{i}:=$ $\mathcal{F}^{-1}\left[\left(\varphi_{i}^{*} h\right) \odot\left(\varphi_{i} \widehat{f}\right)\right] \in \mathcal{S}^{\prime}\left(\mathbb{R}^{d}\right)$. This is well-defined since (5.10) implies $\varphi_{i} h \in \mathcal{F} L^{1}$, and $\varphi_{i}^{*} h=\sum_{\ell \in i^{*}} \varphi_{\ell} h \in \mathcal{F} L^{1}\left(\mathbb{R}^{d}\right)$.

Since $\mathcal{F}: Z^{\prime}(\mathcal{O}) \rightarrow \mathcal{D}^{\prime}(\mathcal{O})$ is an isomorphism, it is enough to show that the series $\sum_{i \in I} \mathcal{F} \psi_{i}$ converges unconditionally in $\mathcal{D}^{\prime}(\mathcal{O})$. To see this, note that $\operatorname{supp} \widehat{\psi_{i}} \subset \operatorname{supp} \varphi_{i} \subset \overline{Q_{i}}$ for all $i \in I$, by Property (iv) of Lemma C.1. Therefore, $\sum_{i \in I} \mathcal{F} \psi_{i}$ converges unconditionally in $\mathcal{D}^{\prime}(\mathcal{O})$ as a locally finite ${ }^{1}$ sum of (tempered) distributions.

(ii) As above, let $\psi_{i}^{(n)}:=\mathcal{F}^{-1}\left[\left(\varphi_{i}^{*} h\right) \odot\left(\varphi_{i} \widehat{f_{n}}\right)\right]$. Note that $\widehat{f}_{n} \rightarrow \widehat{f}$ in $\mathcal{D}^{\prime}(\mathcal{O})$, since $f_{n} \rightarrow f$ in $Z^{\prime}(\mathcal{O})$. Thus, setting $e_{x}: \widehat{\mathbb{R}}^{d} \rightarrow \mathbb{C}, \xi \mapsto e^{2 \pi i\langle x, \xi\rangle}$ for $x \in \mathbb{R}^{d}$, an application of [54,Theorem 7.23] shows that

$$
\mathcal{F}^{-1}\left(\varphi_{i} \widehat{f}\right)(x)=\left(\varphi_{i} \widehat{f}\right)\left(e_{x}\right)=\widehat{f}\left(\varphi_{i} e_{x}\right)=\lim _{n \rightarrow \infty} \widehat{f}_{n}\left(\varphi_{i} e_{x}\right)=\lim _{n \rightarrow \infty} \mathcal{F}^{-1}\left(\varphi_{i} \widehat{f}_{n}\right)(x)
$$

for all $i \in I$ and $x \in \mathbb{R}^{d}$. Therefore, using that $\langle F * G, \varphi\rangle_{\mathcal{S}^{\prime}, \mathcal{S}}=\int_{\mathbb{R}^{d}} G(x) \cdot(\varphi *$ $\widetilde{F})(x) d x$ with $\widetilde{F}(x)=F(-x)$ for $F \in L^{1}, G \in L^{p}$, and the estimate $\left|\mathcal{F}^{-1}\left(\varphi_{i} \widehat{f}_{n}\right)\right| \leq$ $F_{i} \in L^{p}\left(\mathbb{R}^{d}\right)$, we get by the dominated convergence theorem

$$
\begin{aligned}
\left\langle\psi_{i}^{(n)}, \varphi\right\rangle_{\mathcal{S}^{\prime}, \mathcal{S}} & =\left\langle\mathcal{F}^{-1}\left(\varphi_{i}^{*} h\right) * \mathcal{F}^{-1}\left(\varphi_{i} \widehat{f}_{n}\right), \varphi\right\rangle_{\mathcal{S}^{\prime}, \mathcal{S}} \\
& =\int_{\mathbb{R}^{d}} \mathcal{F}^{-1}\left(\varphi_{i} \widehat{f}_{n}\right)(x) \cdot\left(\varphi * \widehat{\varphi_{i}^{*} h}\right)(x) d x \\
& \underset{n \rightarrow \infty}{\longrightarrow} \int_{\mathbb{R}^{d}} \mathcal{F}^{-1}\left(\varphi_{i} \widehat{f}\right)(x) \cdot\left(\varphi * \widehat{\varphi_{i}^{*} h}\right)(x) d x=\left\langle\psi_{i}, \varphi\right\rangle_{\mathcal{S}^{\prime}, \mathcal{S}}
\end{aligned}
$$

for all $\varphi \in \mathcal{S}\left(\mathbb{R}^{d}\right)$ and $i \in I$. Here, we used that $\varphi * \widehat{\varphi_{i}^{*} h} \in L^{1}\left(\mathbb{R}^{d}\right) \cap L^{\infty}\left(\mathbb{R}^{d}\right) \subset$ $L^{p^{\prime}}\left(\mathbb{R}^{d}\right)$.

Now, let $\varphi \in Z(\mathcal{O})$ be arbitrary, so that $\mathcal{F}^{-1} \varphi \in C_{c}^{\infty}(\mathcal{O})$. Then there is a finite set $I_{\varphi} \subset I$ such that $\operatorname{supp} \mathcal{F}^{-1} \varphi \subset{\overline{Q_{i}}}^{c}$ for all $i \in I \backslash I_{\varphi}$. Since $\operatorname{supp} \mathcal{F} \psi_{i} \subset \overline{Q_{i}}$

\footnotetext{
${ }^{1}$ Here, we use that if $\xi_{0} \in \mathcal{O}$ is arbitrary, then $\xi_{0} \in Q_{\ell}$ for some $\ell \in I$ and hence $\varphi_{\ell}^{*}\left(\xi_{0}\right)=1$. Thus, $U:=\left\{\xi \in \mathcal{O}:\left|\varphi_{\ell}^{*}(\xi)\right|>1 / 2\right\} \subset Q_{\ell}^{*}$ is an open neighborhood of $\xi_{0}$; finally, if $U \cap \overline{Q_{i}} \neq \emptyset$, then also $U \cap Q_{i} \neq \emptyset$ and hence $i \in \ell^{* *}=\bigcup_{j \in \ell^{*}} j^{*}$, proving that the family $\left(\overline{Q_{i}}\right)_{i \in I}$ is locally finite on $\mathcal{O}$.
} 
and supp $\mathcal{F} \psi_{i}^{(n)} \subset \overline{Q_{i}}$, this implies $\left\langle\psi_{i}, \varphi\right\rangle_{Z^{\prime}, Z}=\left\langle\mathcal{F} \psi_{i}, \mathcal{F}^{-1} \varphi\right\rangle_{\mathcal{D}^{\prime}, C_{c}^{\infty}}=0$ for all $i \in I \backslash I_{\varphi}$. The same holds for $\psi_{i}$ replaced by $\psi_{i}^{(n)}$. Thus,

$$
\left\langle\Phi_{h} f_{n}, \varphi\right\rangle_{Z^{\prime}, Z}=\sum_{i \in I_{\varphi}}\left\langle\psi_{i}^{(n)}, \varphi\right\rangle_{\mathcal{S}^{\prime}, \mathcal{S}} \underset{n \rightarrow \infty}{\longrightarrow} \sum_{i \in I_{\varphi}}\left\langle\psi_{i}, \varphi\right\rangle_{\mathcal{S}^{\prime}, \mathcal{S}}=\left\langle\Phi_{h} f, \varphi\right\rangle_{Z^{\prime}, Z}
$$

This shows that $\Phi_{h} f_{n} \rightarrow \Phi_{h} f$ with convergence in $Z^{\prime}(\mathcal{O})$.

Finally, we see for $\ell \in I$ directly by definition of $\psi_{i}^{(n)}$ and by definition of the "extended multiplication" $\odot$ that

$$
\begin{aligned}
\mathcal{F}^{-1}\left(\varphi_{\ell} \widehat{\psi_{i}^{(n)}}\right) & =\mathcal{F}^{-1} \varphi_{\ell} * \mathcal{F}^{-1}\left(\varphi_{i}^{*} h\right) * \mathcal{F}^{-1}\left(\varphi_{i} \widehat{f}_{n}\right)=\mathcal{F}^{-1}\left(\varphi_{i}^{*} h\right) * \mathcal{F}^{-1}\left(\varphi_{i} \varphi_{\ell} \widehat{f}_{n}\right) \\
& =\mathcal{F}^{-1}\left(\varphi_{i}^{*} h\right) * \mathcal{F}^{-1}\left(\varphi_{i}\right) * \mathcal{F}^{-1}\left(\varphi_{\ell} \widehat{f}_{n}\right)
\end{aligned}
$$

This shows that $\mathcal{F}^{-1}\left(\varphi_{\ell} \widehat{\psi_{i}^{(n)}}\right)=\mathcal{F}^{-1}\left(\varphi_{i}^{*} h\right) * \mathcal{F}^{-1}\left(\varphi_{i} \varphi_{\ell} \widehat{f_{n}}\right)=0$ if $\ell \in I \backslash i^{*}$, since then $\varphi_{i} \varphi_{\ell} \equiv 0$. Therefore, since $\left|\mathcal{F}^{-1}\left(\varphi_{\ell} \widehat{f}_{n}\right)\right| \leq F_{\ell}$, we see

$$
\begin{aligned}
\left|\mathcal{F}^{-1}\left(\varphi_{\ell} \cdot \widehat{\Phi_{h} f_{n}}\right)\right| & \leq \sum_{i \in \ell^{*}}\left|\mathcal{F}^{-1}\left(\varphi_{\ell} \cdot \widehat{\psi_{i}^{(n)}}\right)\right| \\
& \leq \sum_{i \in \ell^{*}}\left|\mathcal{F}^{-1}\left(\varphi_{i}^{*} h\right)\right| *\left|\mathcal{F}^{-1}\left(\varphi_{i}\right)\right| *\left|\mathcal{F}^{-1}\left(\varphi_{\ell} \widehat{f_{n}}\right)\right| \\
& \leq \sum_{i \in \ell^{*}}\left|\mathcal{F}^{-1}\left(\varphi_{i}^{*} h\right)\right| *\left|\mathcal{F}^{-1}\left(\varphi_{i}\right)\right| * F_{\ell}=: G_{\ell} .
\end{aligned}
$$

In view of Young's inequality, we see

$$
\left\|G_{\ell}\right\|_{L^{p}} \leq \sum_{i \in \ell^{*}}\left\|\mathcal{F}^{-1}\left(\varphi_{i}^{*} h\right)\right\|_{L^{1}}\left\|\mathcal{F}^{-1}\left(\varphi_{i}\right)\right\|_{L^{1}}\left\|F_{\ell}\right\|_{L^{p}} \leq N_{\mathcal{Q}}^{2} C_{\Phi} C_{h} \cdot\left\|F_{\ell}\right\|_{L^{p}}
$$

and hence $\|G\|_{\ell_{w}^{q}\left(I ; L^{p}\right)} \leq N_{\mathcal{Q}}^{2} C_{\Phi} C_{h} \cdot\|F\|_{\ell_{w}^{q}\left(I ; L^{p}\right)}<\infty$, so that indeed each $\Phi_{h} f_{n}$ is $(G, \Phi)$-dominated.

(i) By applying Property (ii) to the constant sequence given by $f_{n}=f$ for all $n \in \mathbb{N}$ and with $F_{i}:=\left|\mathcal{F}^{-1}\left(\varphi_{i} \widehat{f}\right)\right|$, we see that $\Phi_{h} f$ is $(G, \Phi)$-dominated for a function $G \in \ell_{w}^{q}\left(I ; L^{p}\right)$ satisfying $\|G\|_{\ell_{w}^{q}\left(I ; L^{p}\right)} \leq N_{\mathcal{Q}}^{2} C_{\Phi} C_{h} \cdot\|F\|_{\ell_{w}^{q}\left(I ; L^{p}\right)}=N_{\mathcal{Q}}^{2} C_{\Phi} C_{h}$. $\|f\|_{\mathcal{D}\left(\mathcal{Q}, L^{p}, \ell_{w}^{q}\right)}$. This proves the claim.

(iii) If $\widehat{f} \in C_{c}(\mathcal{O})$, then $\varphi_{i} \widehat{f} \in C_{c}(\mathcal{O}) \subset L^{2}\left(\widehat{\mathbb{R}}^{d}\right)$, so that $\left(\varphi_{i}^{*} h\right) \odot\left(\varphi_{i} \widehat{f}\right)=\left(\varphi_{i}^{*} h\right)$. $\left(\varphi_{i} \widehat{f}\right)=\varphi_{i} \cdot h \widehat{f}$; see Lemma C.1(iii). Since $h \widehat{f} \in C_{c}(\mathcal{O})$, it follows $h \widehat{f}=\sum_{i \in I}\left[\varphi_{i}\right.$. $h \widehat{f}]$, where only finitely many terms do not vanish. Hence, by definition of $\Phi_{h} f$,

$$
\Phi_{h} f=\sum_{i \in I} \mathcal{F}^{-1}\left[\left(\varphi_{i}^{*} h\right) \odot\left(\varphi_{i} \widehat{f}\right)\right]=\mathcal{F}^{-1}\left[\sum_{i \in I} \varphi_{i} \cdot h \widehat{f}\right]=\mathcal{F}^{-1}(h \cdot \widehat{f}) .
$$


(iv) We have

$$
\begin{aligned}
\left\|\mathcal{F}^{-1}\left(\varphi_{i} \cdot(g \cdot h)\right)\right\|_{L^{1}} & =\left\|\mathcal{F}^{-1}\left(\varphi_{i} g \cdot \varphi_{i}^{*} h\right)\right\|_{L^{1}} \leq \sum_{\ell \in i^{*}}\left\|\mathcal{F}^{-1}\left(\varphi_{i} g\right)\right\|_{L^{1}} \cdot\left\|\mathcal{F}^{-1}\left(\varphi_{\ell} h\right)\right\|_{L^{1}} \\
& \leq N_{\mathcal{Q}} C_{g} C_{h}<\infty
\end{aligned}
$$

so that $g \cdot h$ is tame. Part (iii) shows for $f \in \mathcal{S}_{\mathcal{O}}\left(\mathbb{R}^{d}\right)$ that $\Phi_{g} f=\mathcal{F}^{-1}(g \widehat{f})$, which in particular implies $\mathcal{F}\left[\Phi_{g} f\right] \in C_{c}(\mathcal{O})$. Thus, by Part (iii) again, $\Phi_{h} \Phi_{g} f=$ $\mathcal{F}^{-1}\left[h \cdot \mathcal{F}\left[\Phi_{g} f\right]\right]=\mathcal{F}^{-1}(h g \widehat{f})=\Phi_{g h} f$. Finally, for arbitrary $f \in \mathcal{D}\left(\mathcal{Q}, L^{p}, \ell_{w}^{q}\right)$, Proposition 3.13 yields a sequence $\left(f_{n}\right)_{n \in \mathbb{N}} \subset \mathcal{S}_{\mathcal{O}}\left(\mathbb{R}^{d}\right)$ which is $(F, \Phi)$-dominated for some $F \in \ell_{w}^{q}\left(I ; L^{p}\right)$ and such that $f_{n} \rightarrow f$ in $Z^{\prime}(\mathcal{O})$. By Part (ii), this implies $\Phi_{g h} f_{n} \rightarrow \Phi_{g h} f$ and $\Phi_{g} f_{n} \rightarrow \Phi_{g} f$ in $Z^{\prime}(\mathcal{O})$. Furthermore, there is $G \in \ell_{w}^{q}\left(I ; L^{p}\right)$ such that each $\Phi_{g} f_{n}$ is $(G, \Phi)$-dominated. Thus, a final application of Part (ii) implies

$$
\Phi_{g h} f=\lim _{n \rightarrow \infty} \Phi_{g h} f_{n}=\lim _{n \rightarrow \infty} \Phi_{h}\left[\Phi_{g} f_{n}\right]=\Phi_{h}\left[\Phi_{g} f\right]
$$

which completes the proof.

\section{Appendix D: Other Auxiliary Results}

\section{D.1: An Estimate for the series $\sum_{k \in \mathbb{Z}^{d}}(1+|\eta+A k|)^{-(d+1)}$}

Lemma D.1 For $\eta \in \mathbb{R}^{d}$ and $A \in \operatorname{GL}(d, \mathbb{R})$,

$$
\sum_{k \in \mathbb{Z}^{d}}(1+|\eta+A k|)^{-(d+1)} \leq(d+1) \cdot 2^{1+2 d} \cdot \max \left\{1,\left\|A^{-1}\right\|^{d+1}\right\} .
$$

Proof First, note that the function $\Theta: \mathbb{R}^{d} \rightarrow[0, \infty], x \mapsto \sum_{k \in \mathbb{Z}^{d}}(1+|x+k|)^{-(d+1)}$ is $\mathbb{Z}^{d}$-periodic, and hence $\|\Theta\|_{\text {sup }}=\left\|\left.\Theta\right|_{[0,1)^{d}}\right\|_{\text {sup. }}$. For $x \in[0,1)^{d}$, we have $\|k\|_{\infty} \leq$ $1+\|x+k\|_{\infty} \leq 1+|x+k|$, and thus $1+\|k\|_{\infty} \leq 2(1+|x+k|)$. Therefore, $\Theta(x) \leq 2^{d+1} \cdot \sum_{k \in \mathbb{Z}^{d}}\left(1+\|k\|_{\infty}\right)^{-(d+1)}$. In order to estimate this last term, we rewrite it using [25,Proposition 6.24] as

$$
\sum_{k \in \mathbb{Z}^{d}}\left(1+\|k\|_{\infty}\right)^{-(d+1)}=\int_{0}^{\infty}\left|\left\{k \in \mathbb{Z}^{d}:\left(1+\|k\|_{\infty}\right)^{-(d+1)}>\lambda\right\}\right| d \lambda
$$

Let $f: \mathbb{Z}^{d} \rightarrow(0,1], k \mapsto\left(1+\|k\|_{\infty}\right)^{-(d+1)}$. For $\lambda \geq 1$, clearly $\left\{k \in \mathbb{Z}^{d}: f(k)>\right.$ $\lambda\}=\emptyset$. In contrast, for $\lambda \in(0,1)$,

$$
\begin{aligned}
\left\{k \in \mathbb{Z}^{d}: f(k)>\lambda\right\} & \subset\left\{k \in \mathbb{Z}^{d}:\|k\|_{\infty} \leq \lambda^{-1 /(d+1)}-1\right\} \\
\subset\left\{k \in \mathbb{Z}^{d}: \forall n \in \underline{d}: k_{n} \in\left\{-\left\lfloor\lambda^{-1 /(d+1)}-1\right\rfloor, \ldots,\right.\right. & \left.\left.\left\lfloor\lambda^{-1 /(d+1)}-1\right\rfloor\right\}\right\},
\end{aligned}
$$


and thus $\left|\left\{k \in \mathbb{Z}^{d}: f(k)>\lambda\right\}\right| \leq\left(1+2\left\lfloor\lambda^{-1 /(d+1)}-1\right\rfloor\right)^{d} \leq 2^{d} \cdot \lambda^{-d /(d+1)}$, which implies

$$
\Theta(x) \leq 2^{d+1} \sum_{k \in \mathbb{Z}^{d}}\left(1+\|k\|_{\infty}\right)^{-(d+1)} \leq 2^{1+2 d} \int_{0}^{1} \lambda^{-\frac{d}{d+1}} d \lambda=(d+1) \cdot 2^{1+2 d}
$$

for all $x \in[0,1)^{d}$, whence $\Theta(x) \leq(d+1) \cdot 2^{1+2 d}$ for all $x \in \mathbb{R}^{d}$.

Now, let $A \in \operatorname{GL}(d, \mathbb{R})$ be arbitrary. Then

$$
\begin{aligned}
& 1+\left|k+A^{-1} \eta\right| \leq 1+\left\|A^{-1}\right\| \cdot\left|A\left(k+A^{-1} \eta\right)\right| \leq \max \left\{1,\left\|A^{-1}\right\|\right\} \\
& \quad\left(1+\left|A\left(k+A^{-1} \eta\right)\right|\right)
\end{aligned}
$$

and hence $(1+|\eta+A k|)^{-(d+1)}=\left(1+\left|A\left(k+A^{-1} \eta\right)\right|\right)^{-(d+1)} \leq \max \left\{1,\left\|A^{-1}\right\|^{d+1}\right\}$. $\left(1+\left|k+A^{-1} \eta\right|\right)^{-(d+1)}$. Overall, we see for arbitrary $\eta \in \mathbb{R}^{d}$ and $A \in \operatorname{GL}(d, \mathbb{R})$ that

$$
\begin{aligned}
\sum_{k \in \mathbb{Z}^{d}}(1+|\eta+A k|)^{-(d+1)} & \leq \max \left\{1,\left\|A^{-1}\right\|^{d+1}\right\} \cdot \sum_{k \in \mathbb{Z}^{d}}\left(1+\left|k+A^{-1} \eta\right|\right)^{-(d+1)} \\
& =\max \left\{1,\left\|A^{-1}\right\|^{d+1}\right\} \cdot \Theta\left(A^{-1} \eta\right) \\
& \leq(d+1) \cdot 2^{1+2 d} \cdot \max \left\{1,\left\|A^{-1}\right\|^{d+1}\right\}
\end{aligned}
$$

finishing the proof.

As a corollary, we get the following estimate for the series where we sum over $k \in \mathbb{Z}^{d} \backslash\{0\}$ instead of $k \in \mathbb{Z}^{d}$.

Corollary D.2 For $\eta \in \mathbb{R}^{d}$ and $A \in \operatorname{GL}(d, \mathbb{R})$, we have

$$
\sum_{k \in \mathbb{Z}^{d} \backslash\{0\}}(1+|\eta+A k|)^{-(d+1)} \leq(d+1) \cdot 2^{3+4 d} \cdot(1+|\eta|) \cdot \max \left\{\left\|A^{-1}\right\|,\left\|A^{-1}\right\|^{d+1}\right\}
$$

Proof We distinguish two cases.

First, suppose $\left|A^{-1} \eta\right| \leq \frac{1}{3}$. Then, noting that $|k| \geq 1$ for all $k \in \mathbb{Z}^{d} \backslash\{0\}$, we get the estimate $\left|k+A^{-1} \eta\right| \geq|k|-\left|A^{-1} \eta\right| \geq \frac{|k|}{2}+\frac{1}{2}-\left|A^{-1} \eta\right| \geq \frac{|k|}{2} \geq \frac{1+|k|}{4}$. Next, note that $|x|=\left|A^{-1} A x\right| \leq\left\|A^{-1}\right\||A x|$, and hence $|A x| \geq\left\|A^{-1}\right\|^{-1}|x|$ for all $x \in \mathbb{R}^{d}$. This implies

$$
1+|\eta+A k| \geq|A k+\eta| \geq\left\|A^{-1}\right\|^{-1} \cdot\left|k+A^{-1} \eta\right| \geq \frac{\left\|A^{-1}\right\|^{-1}}{4} \cdot(1+|k|) .
$$


Now, Lemma D.1 shows that

$$
\begin{aligned}
\sum_{k \in \mathbb{Z}^{d} \backslash\{0\}}(1+|\eta+A k|)^{-(d+1)} & \leq 4^{d+1}\left\|A^{-1}\right\|^{d+1} \cdot \sum_{k \in \mathbb{Z}^{d} \backslash\{0\}}(1+|k|)^{-(d+1)} \\
& \leq(d+1) \cdot 2^{3+4 d} \cdot\left\|A^{-1}\right\|^{d+1} \\
& \leq(d+1) \cdot 2^{3+4 d} \cdot(1+|\eta|) \cdot \max \left\{\left\|A^{-1}\right\|,\left\|A^{-1}\right\|^{d+1}\right\} .
\end{aligned}
$$

For the other case, suppose $\left|A^{-1} \eta\right|>\frac{1}{3}$. Then $(1+|\eta|)\left\|A^{-1}\right\| \geq\left\|A^{-1}\right\| \cdot|\eta| \geq$ $\left|A^{-1} \eta\right|>\frac{1}{3}$, and

$$
\begin{aligned}
\max \left\{1,\left\|A^{-1}\right\|^{d+1}\right\} & \leq \max \left\{3(1+|\eta|)\left\|A^{-1}\right\|,\left\|A^{-1}\right\|^{d+1}\right\} \\
& \leq 4(1+|\eta|) \cdot \max \left\{\left\|A^{-1}\right\|,\left\|A^{-1}\right\|^{d+1}\right\} .
\end{aligned}
$$

Now, an application of Lemma D.1 shows that

$$
\begin{aligned}
\sum_{k \in \mathbb{Z}^{d} \backslash\{0\}}(1+|\eta+A k|)^{-(d+1)} & \leq(d+1) 2^{1+2 d} \cdot \max \left\{1,\left\|A^{-1}\right\|^{d+1}\right\} \\
& \leq(d+1) 2^{3+2 d} \cdot(1+|\eta|) \cdot \max \left\{\left\|A^{-1}\right\|,\left\|A^{-1}\right\|^{d+1}\right\} .
\end{aligned}
$$

Together with the first case, this shows that the claimed estimate always holds.

\section{D.2: Proof of Lemma 7.2}

For brevity, set $\langle\langle\xi\rangle\rangle:=1+|\xi|^{2}$ for $\xi \in \widehat{\mathbb{R}}^{d}$. With this notation, [62,Lemma 6.8] shows for arbitrary $\theta \in \mathbb{R}$ and $\alpha \in \mathbb{N}_{0}^{d}$ that there is a polynomial $P_{\theta, \alpha} \in \mathbb{R}\left[\xi_{1}, \ldots, \xi_{d}\right]$ such that, for all $\xi \in \widehat{\mathbb{R}}^{d}$,

$$
\partial^{\alpha}\langle\langle\xi\rangle\rangle^{\theta}=\langle\langle\xi\rangle\rangle^{\theta-|\alpha|} \cdot P_{\theta, \alpha}(\xi) \quad \text { and } \quad\left|P_{\theta, \alpha}(\xi)\right| \leq C_{\theta, \alpha} \cdot(1+|\xi|)^{|\alpha|}
$$

where $C_{\theta, \alpha}=|\alpha| ! \cdot[2(1+d+|\theta|)]^{|\alpha|}$. Since $(1+|\xi|)^{k} \leq 2^{k} \cdot\langle\langle\xi\rangle\rangle^{k / 2}$ for all $k \geq 0$, it follows that

$$
(1+|\xi|)^{|\alpha|} \cdot\langle\langle\xi\rangle\rangle^{\theta-|\alpha|} \leq 2^{|\alpha|} \cdot\langle\langle\xi\rangle\rangle^{\theta-|\alpha| / 2} \leq 2^{|\alpha|} \cdot\langle\langle\xi\rangle\rangle^{\theta}
$$

for all $\xi \in \widehat{\mathbb{R}}^{d}, \theta \in \mathbb{R}$ and $\alpha \in \mathbb{N}_{0}^{d}$. Next, for $\theta=-\frac{1}{2}(d+1)$ and any $\alpha \in \mathbb{N}_{0}^{d}$ with $|\alpha| \leq d+1$

$$
\begin{aligned}
C_{-(d+1) / 2, \alpha}=|\alpha| ! \cdot\left[2\left(1+d+\left|-\frac{d+1}{2}\right|\right)\right]^{|\alpha|} & \leq(d+1) ! \cdot[3 \cdot(d+1)]^{|\alpha|} \\
& \leq\left(3 \cdot(d+1)^{2}\right)^{d+1} .
\end{aligned}
$$


Combining Equations (D.2) and (D.3) with the elementary estimate $1+|\xi| \leq 2\langle\langle\xi\rangle\rangle^{1 / 2}$, we see that

$$
\begin{aligned}
\max _{|\alpha| \leq d+1}\left|\partial^{\alpha} h_{2}(\xi)\right| & =\max _{|\alpha| \leq d+1}\left|\partial^{\alpha}\langle\langle\xi\rangle\rangle^{-(d+1) / 2}\right| \\
& \leq\left(3(d+1)^{2}\right)^{d+1} \max _{|\alpha| \leq d+1}(1+|\xi|)^{|\alpha|}\langle\langle\xi\rangle\rangle^{-\frac{d+1}{2}-|\alpha|} \\
& \leq\left(6(d+1)^{2}\right)^{d+1}\langle\langle\xi\rangle\rangle^{-\frac{d+1}{2}} \leq C^{\prime} \cdot(1+|\xi|)^{-(d+1)}
\end{aligned}
$$

For the estimate concerning $h_{1}$, note that since $C_{\theta, \alpha}=C_{-\theta, \alpha}$, we also have $C_{(d+1) / 2, \beta} \leq\left(3 \cdot(d+1)^{2}\right)^{d+1}$ for all $\beta \in \mathbb{N}_{0}^{d}$ with $|\beta| \leq d+1$. Hence, using the Leibniz rule and Equations (D.2) and (D.3), it follows for arbitrary $\xi \in \widehat{\mathbb{R}}^{d}$ that

$$
\begin{aligned}
\max _{|\alpha| \leq d+1}\left|\partial^{\alpha} h_{1}(\xi)\right| & \leq \sum_{\beta \leq \alpha}\left(\begin{array}{c}
\alpha \\
\beta
\end{array}\right)\left|\partial^{\beta}\langle\langle\xi\rangle\rangle^{(d+1) / 2}\right| \cdot\left|\partial^{\alpha-\beta} g(\xi)\right| \\
& \leq \varrho(\xi) \cdot(1+|\xi|)^{-(d+1)} \sum_{\beta \leq \alpha}\left(\begin{array}{c}
\alpha \\
\beta
\end{array}\right) C_{(d+1) / 2, \beta}(1+|\xi|)^{|\beta|}\langle\langle\xi\rangle\rangle^{\frac{d+1}{2}-|\beta|} \\
& \leq\left(6 \cdot(d+1)^{2}\right)^{d+1} \varrho(\xi)(1+|\xi|)^{-(d+1)}\langle\langle\xi\rangle\rangle^{(d+1) / 2} \sum_{\beta \leq \alpha}\left(\begin{array}{c}
\alpha \\
\beta
\end{array}\right) \\
& \leq C^{\prime} \varrho(\xi),
\end{aligned}
$$

which completes the proof.

\section{References}

1. Aldroubi, A., Gröchenig, K.: Nonuniform sampling and reconstruction in shift-invariant spaces. SIAM Rev. 43(4), 585-620 (2001)

2. Aldroubi, A., Cabrelli, C., Molter, U.M.: Wavelets on irregular grids with arbitrary dilation matrices and frame atoms for $L^{2}\left(\mathbb{R}^{d}\right)$. Appl. Comput. Harmon. Anal. 17(2), 119-140 (2004)

3. Alt, H.: Linear Functional Analysis. Springer, London (2016)

4. Balan, R., Casazza, P.G., Heil, C., Landau, Z.: Density, overcompleteness, and localization of frames. I. Theory. J. Fourier Anal. Appl. 12(2), 105-143 (2006)

5. Balan, R., Casazza, P.G., Heil, C., Landau, Z.: Density, overcompleteness, and localization of frames. II. Gabor systems. J. Fourier Anal. Appl. 12(3), 309-344 (2006)

6. Balan, R., Christensen, J.G., Krishtal, I.A., Okoudjou, K.A., Romero, J.L.: Multi-window Gabor frames in amalgam spaces. Math. Res. Lett. 21(1), 55-69 (2014)

7. Balazs, P.: Basic definition and properties of Bessel multipliers. J. Math. Anal. Appl. 325(1), 571-585 (2007)

8. Barrios, B., Betancor, J.J.: Characterizations of anisotropic Besov spaces. Math. Nachr. 284(14-15), 1796-1819 (2011)

9. Berend, D., Tassa, T.: Improved bounds on Bell numbers and on moments of sums of random variables. Probab. Math. Stat. 30(2), 185-205 (2010)

10. Borup, L., Nielsen, M.: Banach frames for multivariate $\alpha$-modulation spaces. J. Math. Anal. Appl. 321(2), 880-895 (2006)

11. Borup, L., Nielsen, M.: Frame decomposition of decomposition spaces. J. Fourier Anal. Appl. 13(1), 39-70 (2007)

12. Bownik, M.: Anisotropic Hardy spaces and wavelets. Mem. Am. Math. Soc. 164(781), vi+122 (2003) 
13. Bownik, M.: Atomic and molecular decompositions of anisotropic Besov spaces. Math. Z. 250(3), 539-571 (2005)

14. Bui, H.-Q., Laugesen, R.S.: Affine synthesis onto Lebesgue and Hardy spaces. Indiana Univ. Math. J. 57(5), 2203-2233 (2008)

15. Bui, H.-Q., Laugesen, R.S.: Wavelet frame bijectivity on Lebesgue and Hardy spaces. J. Fourier Anal. Appl. 19(2), 376-409 (2013)

16. Cabrelli, C., Molter, U., Romero, J.L.: Non-uniform painless decompositions for anisotropic Besov and Triebel-Lizorkin spaces. Adv. Math. 232, 98-120 (2013)

17. Christensen, O., Rahimi, A.: Frame properties of wave packet systems in $L^{2}\left(\mathbb{R}^{d}\right)$. Adv. Comput. Math. 29(2), 101-111 (2008)

18. Christensen, O., Hasannasab, M., Lemvig, J.: Explicit constructions and properties of generalized shift-invariant systems in $L^{2}(\mathbb{R})$. Adv. Comput. Math. 43(2), 443-472 (2017)

19. Daubechies, I.: Ten lectures on wavelets, volume 61 of CBMS-NSF Regional Conference Series in Applied Mathematics. Society for Industrial and Applied Mathematics (SIAM), Philadelphia, PA (1992)

20. de Hoop, M.V., Gröchenig, K., Romero, J.L.: Exact and approximate expansions with pure Gaussian wave packets. SIAM J. Math. Anal. 46(3), 2229-2253 (2014)

21. Feichtinger, H.G.: Banach convolution algebras of Wiener type. In: Functions, series, operators, Vol. I, II (Budapest, 1980). Colloq. Math. Soc. János Bolyai, vol. 35, pages 509-524. North-Holland, Amsterdam (1983)

22. Feichtinger, H.G.: Atomic characterizations of modulation spaces through Gabor-type representations. Rocky Mountain J. Math. 19(1), 113-125 (1989)

23. Feichtinger, H.G., Gröbner, P.: Banach spaces of distributions defined by decomposition methods. I. Math. Nachr. 123, 97-120 (1985)

24. Feichtinger, H.G., Gröchenig, K.H.: Banach spaces related to integrable group representations and their atomic decompositions. I. J. Funct. Anal. 86(2), 307-340 (1989)

25. Folland, G.: Real Analysis: Modern Techniques and Their Applications. Pure and Applied Mathematics, vol. 2. Wiley, New York (1999)

26. Fournier, J.J.F., Stewart, J.: Amalgams of $L^{p}$ and $l^{q}$. Bull. Am. Math. Soc. (N.S.) 13(1), 1-21 (1985)

27. Frazier, M., Jawerth, B.: Decomposition of Besov spaces. Indiana Univ. Math. J. 34(4), 777-799 (1985)

28. Frazier, M., Jawerth, B.: A discrete transform and decompositions of distribution spaces. J. Funct. Anal. 93(1), 34-170 (1990)

29. Frazier, M., Jawerth, B., Weiss, G.: Littlewood-Paley theory and the study of function spaces. In: CBMS Regional Conference Series in Mathematics, vol. 79. Published for the Conference Board of the Mathematical Sciences, Washington (1991)

30. Führ, H., Lemvig, J.: System bandwidth and the existence of generalized shift-invariant frames. J. Funct. Anal. 276(2), 563-601 (2019)

31. Führ, H., Voigtlaender, F.: Wavelet coorbit spaces viewed as decomposition spaces. J. Funct. Anal. 269, 80-154 (2015)

32. Gröbner, P.: Banachräume glatter Funktionen und Zerlegungsmethoden. PhD thesis, University of Vienna (1992)

33. Gröchenig, K.: Describing functions: atomic decompositions versus frames. Monatsh. Math. 112(1), $1-42$ (1991)

34. Gröchenig, K.: Foundations of Time-Frequency Analysis. Applied and Numerical Harmonic Analysis. Birkhäuser, Boston (2001)

35. Gröchenig, K.: Localization of frames, Banach frames, and the invertibility of the frame operator. J. Fourier Anal. Appl. 10(2), 105-132 (2004)

36. Gröchenig, K., Klotz, A.: Norm-controlled inversion in smooth Banach algebras. II. Math. Nachr. 287(8-9), 917-937 (2014)

37. Gröchenig, K., Samarah, S.: Nonlinear approximation with local Fourier bases. Constr. Approx. 16(3), 317-331 (2000)

38. Heil, C.E., Walnut, D.F.: Continuous and discrete wavelet transforms. SIAM Rev. 31(4), 628-666 (1989)

39. Hernández, E., Labate, D., Weiss, G.: A unified characterization of reproducing systems generated by a finite family. II. J. Geom. Anal. 12(4), 615-662 (2002)

40. Holland, F.: Harmonic analysis on amalgams of $L^{p}$ and $l^{q}$. J. Lond. Math. Soc. 2(10), 295-305 (1975) 
41. Jakobsen, M., Lemvig, J.: Reproducing formulas for generalized translation invariant systems on locally compact abelian groups. Trans. Am. Math. Soc. 368, 8447-8480 (2016)

42. Johnson, W.P.: The curious history of Faà di Bruno's formula. Am. Math. Mon. 109(3), 217-234 (2002)

43. Krishtal, I.A., Okoudjou, K.A.: Invertibility of the Gabor frame operator on the Wiener amalgam space. J. Approx. Theory 153(2), 212-224 (2008)

44. Kyriazis, G., Petrushev, P.: New bases for Triebel-Lizorkin and Besov spaces. Trans. Am. Math. Soc. 354(2), 749-776 (2002)

45. Lemvig, J., Van Velthoven, J.T.: Criteria for generalized translation-invariant frames. Stud. Math. 251(1), 31-63 (2020)

46. Meyer, Y.: Wavelets and operators. Cambridge Studies in Advanced Mathematics, vol. 37. Cambridge University Press, Cambridge (1992)

47. Nielsen, M.: Frames for decomposition spaces generated by a single function. Collect. Math. $\mathbf{6 5}(2)$, 183-201 (2014)

48. Nielsen, M., Rasmussen, K.N.: Compactly supported frames for decomposition spaces. J. Fourier Anal. Appl. 18(1), 87-117 (2012)

49. Peetre, J.: New Thoughts on Besov Spaces. Duke University Mathematics Series, Mathematics Dept, Duke University, Durham (1976)

50. Robbins, H.: A remark on Stirling's formula. Am. Math. Mon. 62, 26-29 (1955)

51. Romero, J.L.: Explicit localization estimates for spline-type spaces. Sampl. Theory Signal Image Process. 8(3), 249-259 (2009)

52. Romero, J.L.: Surgery of spline-type and molecular frames. J. Fourier Anal. Appl. 17(1), 135-174 (2011)

53. Ron, A., Shen, Z.: Generalized shift-invariant systems. Constr. Approx. 22(1), 1-45 (2005)

54. Rudin, W.: Functional Analysis. McGraw-Hill Education, New York (1973)

55. Stöckert, B., Triebel, H.: Decomposition methods for function spaces of $B_{p, q}^{S}$ type and $F_{p, q}^{s}$ type. Math. Nachr. 89, 247-267 (1979)

56. Triebel, H.: Fourier Analysis and Function Spaces (selected topics). Teubner Verlagsgesellschaft, Leipzig (1977)

57. Triebel, H.: General function spaces. I. Decomposition methods. Math. Nachr. 79, 167-179 (1977)

58. Triebel, H.: Spaces of Besov-Hardy-Sobolev type. BSB B. G. Teubner Verlagsgesellschaft, Leipzig (1978)

59. van Velthoven, J.T.: On the local integrability condition for generalised translation-invariant systems. Collect. Math. 70(3), 407-429 (2019)

60. Voigtlaender, F.: Embeddings of decomposition spaces into Sobolev and BV spaces (2016). arxiv:abs/1601.02201

61. Voigtlaender, F.: Embeddings of decomposition spaces. Mem. Am. Math. Soc. arxiv:1605.09705

62. Voigtlaender, F.: Structured, compactly supported Banach frame decompositions of decomposition spaces. Diss. Math. arxiv: 1612.08772

63. Walnut, D.F.: Continuity properties of the Gabor frame operator. J. Math. Anal. Appl. 165(2), 479-504 (1992)

Publisher's Note Springer Nature remains neutral with regard to jurisdictional claims in published maps and institutional affiliations. 